\title{
The Hyperpolarization-Activated Cyclic Nucleotide-Gated Channels: from Biophysics to Pharmacology of a Unique Family of Ion Channels
}

\author{
Laura Sartiani, Guido Mannaioni, Alessio Masi, Maria Novella Romanelli, and Elisabetta Cerbai \\ Department of Neurosciences, Psychology, Drug Research, and Child Health, University of Florence, Firenze, Italy
}

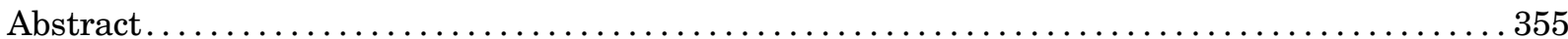

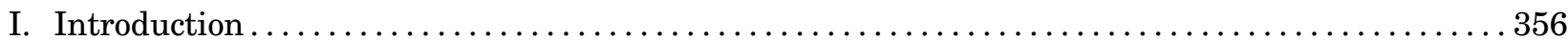

II. Hyperpolarization-Activated Cyclic Nucleotide-Gated Channels: Basic Facts . . . . . . . . . . . . 356

A. Genetic and Molecular Characteristics of the HCN Family . . . . . . . . . . . . . . . . . 356

B. Biophysical Features of HCN Isoforms . . . . . . . . . . . . . . . . . . . . . . . . 357

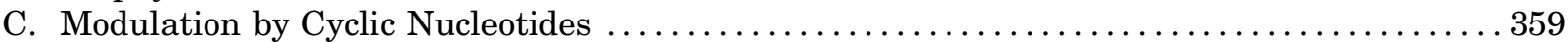

1. Binding of cAMP and Modification of Gating Properties ...................... 359

2. Other Cyclic Nucleotides......................................... 360

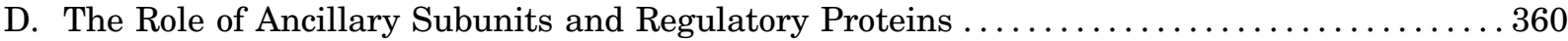

E. From Transcriptional Control to Post-Translational Modifications.................... 361

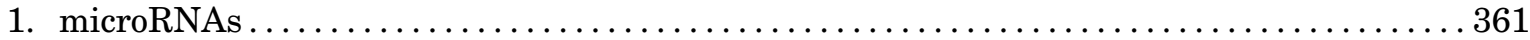

2. Regulation by Membrane Phosphoinositides........................... 362

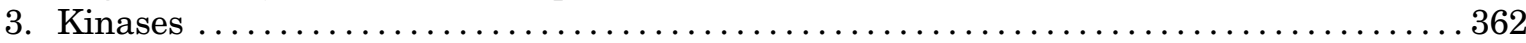

III. Origins, Physiology, and Pathophysiology of HCN Channels . . . . . . . . . . . . . . . . . . 363

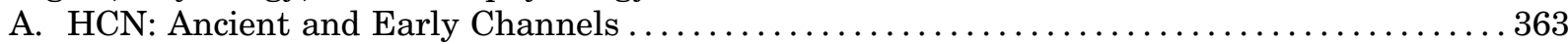

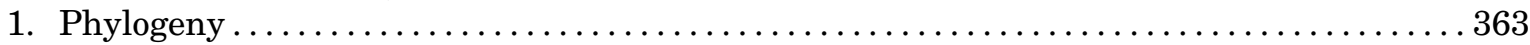

2. HCN Channels in Stem Cells ........................................ 363

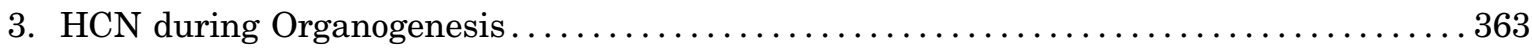

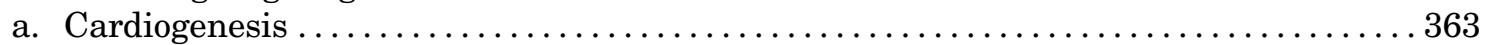

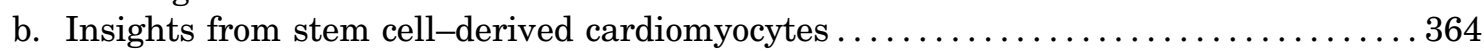

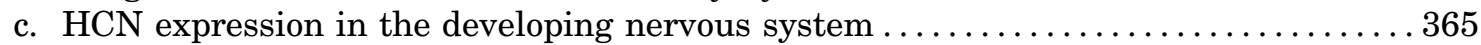

B. Distribution, Adaptive, and Maladaptive Role of HCN Channels in Mammals ........... 365

1. HCN Current in the Heart: an Ideal Pharmacological Target .................... 365

a. Expression and physiologic role of HCN isoforms in cardiac regions . . . . . . . . . 366

i. HCN in SAN Cells and Its Contribution to Cardiac

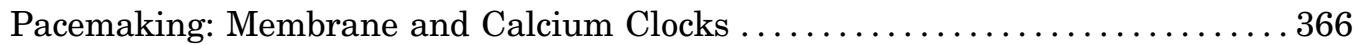

ii. HCN Expression and Function in Subsidiary Pacemakers ................. 367

b. Altered HCN function and cardiac disease ............................ 368

i. Altered HCN Properties and Dysfunction of Sinus and Atrioventricular Nodes . 368

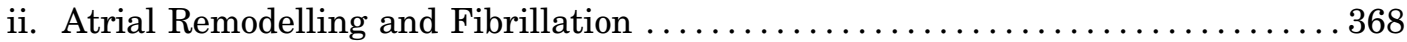

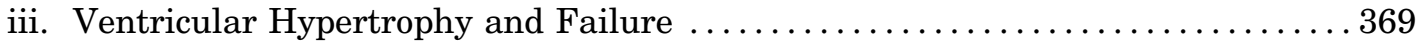

2. HCN in the Central and Peripheral Nervous System ...................... 371

a. Overview of HCN distribution at regional, cellular, and subcellular level ......... 371

b. Physiologic role of HCN channels in the CNS: general aspects................ 372

i. Regulation of Resting Membrane Potential and Intrinsic Excitability .......... 372

A.M. is supported by a fellowship from the Minister of Health (Bando Ricerca Finalizzata e Giovani Ricercatori 2011-2012, GR-201102346829). This work was supported by Ente Cassa di Risparmio di Firenze (Grants 2013.0683A2202.2677 to L.S. and 2013.0102 to G.M.).

L.S. and G.M. are co-first authors.

Address correspondence to: Prof. Elisabetta Cerbai, Department of Neurofarba, University of Florence, Viale G. Pieraccini 6, 50139 Firenze, Italy. E-mail: elisabetta.cerbai@unfi.it

https://doi.org/10.1124/pr.117.014035. 


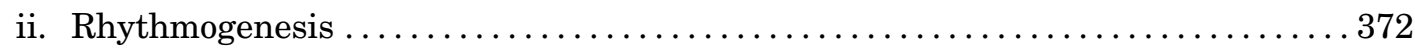

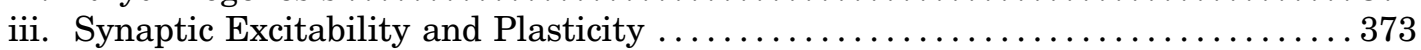

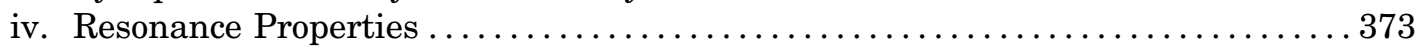

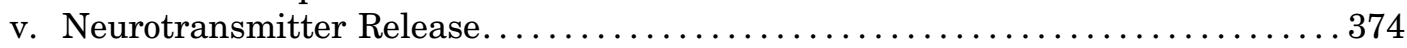

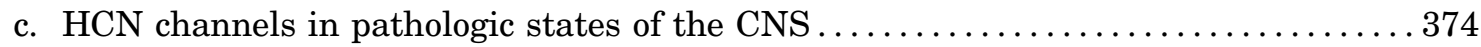

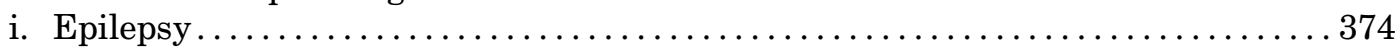

ii. Autism Spectrum Disorder....................................... 374

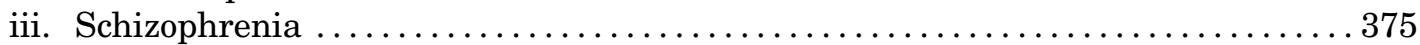

iv. Substances of Abuse and Addiction................................. 375

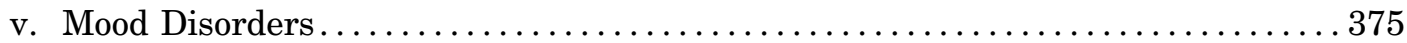

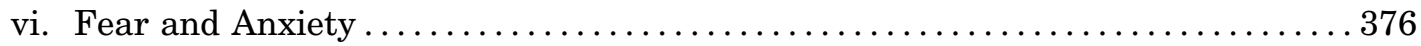

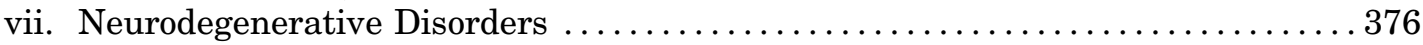

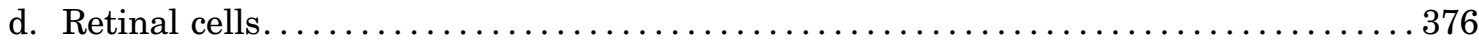

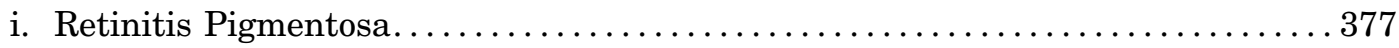

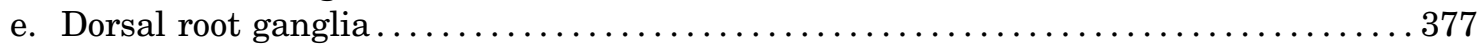

i. Synaptic Transmission in Dorsal Root Ganglion Neurons ................. 377

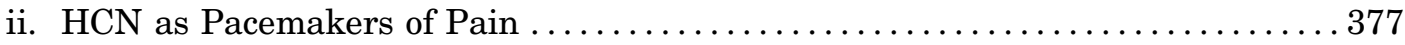

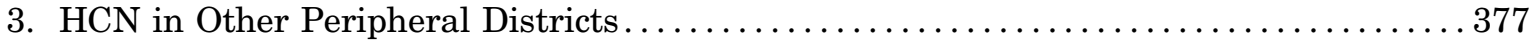

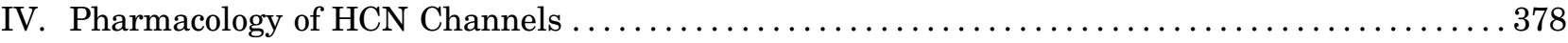

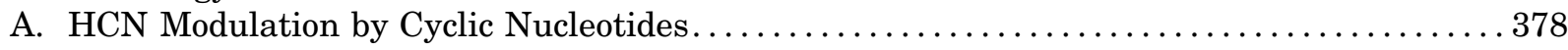

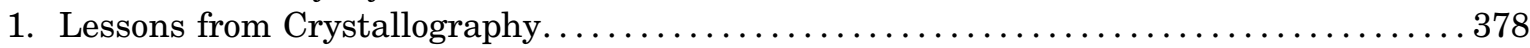

2. Structure-Activity Relationships of Cyclic Nucleotides and Analogs . . . . . . . . . . 379

B. Pharmacology of HCN Blockade by Exogenous Ligands ....................... 380

1. Ivabradine, Cilobradine, and Other Specific Bradycardic Agents ................. 380

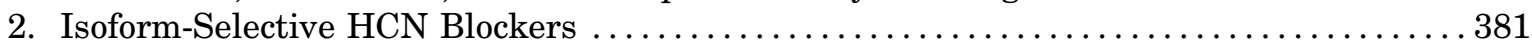

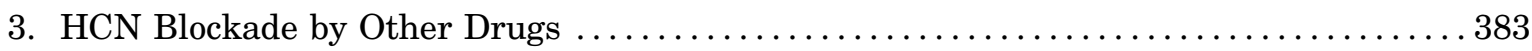

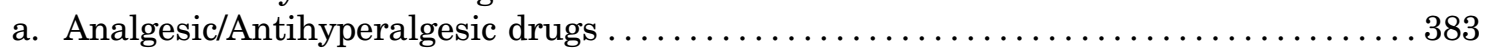

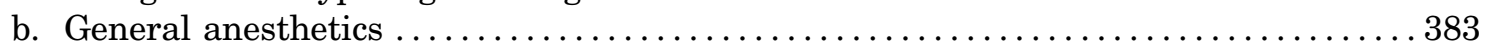

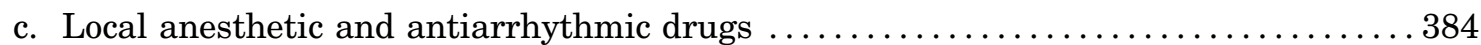

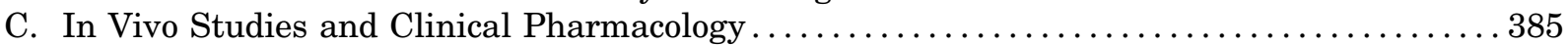

1. Ivabradine in Angina and Heart Failure ............................... 385

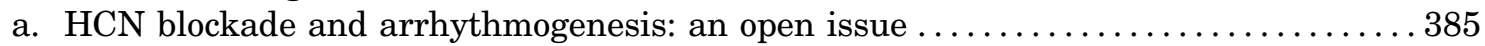

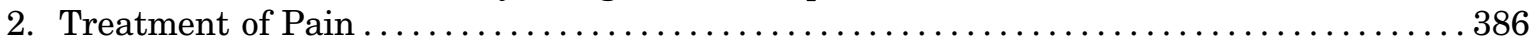

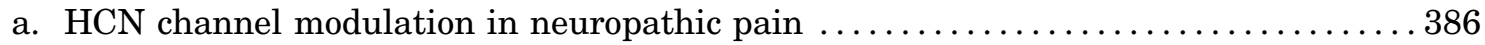

b. Participation of HCN channels in inflammatory pain behavior $\ldots \ldots \ldots \ldots \ldots \ldots \ldots$

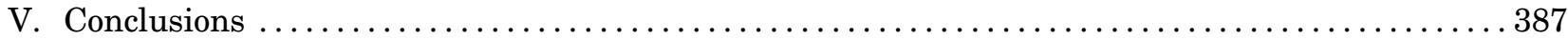

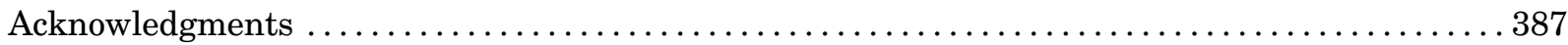

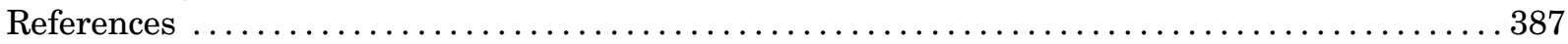

\begin{abstract}
Hyperpolarization-activated, cyclic nucleotide-gated (HCN) channels are important members of the voltage-gated pore loop channels family. They show unique features: they open at hyperpolarizing potential, carry a mixed $\mathrm{Na} / \mathrm{K}$ current, and are regulated by cyclic nucleotides. Four different isoforms have been cloned
\end{abstract}

(HCN1-4) that can assemble to form homo- or heterotetramers, characterized by different biophysical properties. These proteins are widely distributed throughout the body and involved in different physiologic processes, the most important being the generation of spontaneous electrical activity in the heart and the

ABBREVIATIONS: 5-HT, 5-hydroxytryptamine (serotonin); $\alpha_{2}$-AR, $\alpha_{2}$-adrenergic receptor; AF, atrial fibrillation; ANP, atrial natriuretic peptide; AP, action potential; AVN, atrioventricular node; bpm, beats per minute; cCMP, cytidine--3',5'-cyclic monophosphate; cGMP, guanosine-3',5'-cyclic monophosphate; cIMP, inosine 3',5'-cyclic monophosphate; CNBD, cyclic nucleotide binding domain; CNG, cyclic nucleotide gated; CNS, central nervous system; cPMP, purine $3^{\prime}, 5^{\prime}$-cyclic monophosphate; cUMP, uridine- $3^{\prime}, 5^{\prime}$-cyclic monophosphate; D1, dopaminergic receptor type 1; DA, dopaminergic; DDR, diastolic depolarization rate; DRG, dorsal root ganglia; E, embryonic day; EC, entorhinal cortex; EEG, electroencephalogram; EPSP, excitatory postsynaptic potential; HCN, hyperpolarization-activated cyclic nucleotidegated channel; HEK, human embryonic kidney; hHCN, human HCN; $I_{\mathrm{f}}$, funny current; $I_{\mathrm{h}}$, hyperpolarization-activated current; KCR1, K ${ }^{+}$ channel regulator 1; KO, knockout; mHCN, murine HCN; MiR, microRNA; MiRP1, MinK-related peptide $1 ; \mathrm{NCX} \mathrm{Na}^{+} / \mathrm{Ca}^{2+}$ exchanger; $\mathrm{NMDA}^{2}$ $N$-methyl-D-aspartate; NMR, nuclear magnetic resonance; PD, Parkinson disease; PFC, prefrontal cortex; PI(4,5)P2, phosphatidylinositol 4,5bisphosphate; PKA, protein kinase A; PNS, peripheral nervous system; SAN, sinoatrial node; SBA, specific bradycardic agent; SNc, substantia nigra pars compacta; spHCN, sea urchin sperm HCN channel; SUMO, small ubiquitin-like modifier; TRIP8b, tetratricopeptide repeat-containing Rab8b-interacting protein; $\mathrm{V}_{1 / 2}$, voltage of half-maximal activation; VTA, ventral tegmental area; WT, wild type; ZD7288, 4-(N-ethyl-N-phenylamino)-1,2 dimethyl-6(methylamino) pyrimidinium chloride. 
regulation of synaptic transmission in the brain. Their role in heart rate, neuronal pacemaking, dendritic integration, learning and memory, and visual and pain perceptions has been extensively studied; these channels have been found also in some peripheral tissues, where their functions still need to be fully elucidated. Genetic defects and altered expression of HCN channels are linked to several pathologies, which makes these proteins attractive

\section{Introduction}

The family of hyperpolarization-activated, cyclic nucleotide-gated (HCN) channels has attracted increasing attention since the discovery of the coding genes and corresponding proteins, in the late 1990s (Ludwig et al., 1998; Santoro et al., 1998). At that time, the announcement that the molecular fingerprint of the funny current $\left(I_{\mathrm{f}}\right)$ (Brown et al., 1979)-alias hyperpolarization-activated current $\left(I_{\mathrm{h}}\right)$ or queer current (Halliwell and Adams, 1982) — was finally sequenced and generated great excitement in a vast audience of scientists belonging to different disciplines, from cardiology to neurosciences, from biophysics to molecular biology and pharmacology. Prompted by the advancement of molecular, electrophysiological, and optical techniques, a mass of information has been accumulating rapidly and exponentially on the biophysical properties and regulatory features of $\mathrm{HCN}$ isoforms, including their tissue distribution. Many aspects have been reviewed previously in excellent papers (Accili et al., 2002; DiFrancesco and Borer, 2007; Biel et al., 2009; DiFrancesco and DiFrancesco, 2015). Therefore, this review will recall briefly the main tracts of $\mathrm{HCN}$ characteristics, pointing to recent discoveries, and will focus on HCN contribution to cell function and dysfunction, trying to emphasize the potential role of these channels as a target of existing or novel pharmacological approaches.

\section{Hyperpolarization-Activated Cyclic Nucleotide-Gated Channels: Basic Facts}

The hyperpolarization-activated cyclic nucleotidemodulated proteins are voltage-dependent ion channels, conducting both $\mathrm{Na}^{+}$and $\mathrm{K}^{+}$, blocked by millimolar concentrations of extracellular $\mathrm{Cs}^{+}$, and modulated by cyclic nucleotides (mainly cAMP) that contribute crucially to the pacemaker activity in cardiac nodal cells and impulse generation and transmission in neurons (DiFrancesco et al., 1986; Pape and McCormick, 1989; DiFrancesco, 1993; Pape, 1996). Their molecular and functional expression has been also detected in human and animal tissues not canonically classified as excitable and in undifferentiated (e.g., stem) or immature cell types. Altogether, these pieces of information allow speculation that HCN channels play a role beyond pacemaking and raise the interest for $\mathrm{HCN}$ as targets targets for translational research; at the moment only one drug (ivabradine), which specifically blocks the hyperpolarization-activated current, is clinically available. This review discusses current knowledge about HCN channels, starting from their biophysical properties, origin, and developmental features, to (patho)physiologic role in different tissues and pharmacological modulation, ending with their present and future relevance as drug targets. of therapies, including-but not limited to-ivabradine, the only available drug to date acting as a specific bradycardic agent.

Before starting with the fundamental properties of these channels, Brown et al. (1979) should be acknowledged for the first description of a current activated upon hyperpolarization in the sinoatrial node (SAN); they termed the current funny $\left(I_{\mathrm{f}}\right)$ for its peculiar voltage dependence. However, the most general term hyperpolarization-activated current $\left(I_{\mathrm{h}}\right)$ (Yanagihara et al., 1980; Yanagihara and Irisawa, 1980) will be used throughout the text to indicate the current.

\section{A. Genetic and Molecular Characteristics of the HCN Family}

The HCN family consists of four isoforms (HCN1-4). The first three full-length cDNAs encoding for HCN1-3 were identified in the mouse brain (Ludwig et al., 1998; Santoro et al., 1998). A fourth isoform (HCN4) was detected by screening a cDNA library from the human heart, and its expression was found remarkably higher throughout human cardiac tissue (atria and ventricles) than in brain (Ludwig et al., 1999). Afterward, HCN4 was detected also in other tissues, such as thalamus and testis (Seifert et al., 1999). A more detailed description of tissue-specific distribution of HCN isoforms will be given in the next section Distribution, Adaptive, and Maladaptive Role of HCN Channels in Mammals.

The general sequence of HCN genes resembles that of six-transmembrane segment, voltage-activated channel subunits; four subunits assemble in homo- or heterotetramers with a stoichiometry that is not completely defined yet (Chen et al., 2001b; Xue et al., 2002; Altomare et al., 2003; Much et al., 2003; Whitaker et al., 2007; Ye and Nerbonne, 2009). However, HCN channels encompass a unique combination of traits typical of different channel families (Fig. 1).

First, HCNs have a pore region between S5 and S6, highly conserved in all isoforms and very much like to $\mathrm{K}^{+}$-selective voltage-dependent channels $\left(\mathrm{K}_{\mathrm{v}}\right)$ in the amino acid sequence (Robinson and Siegelbaum, 2003; Biel et al., 2009). Despite similarity, HCN channels conduct both $\mathrm{Na}$ and $\mathrm{K}$ ions with a scarce selectivity (DiFrancesco, 1981b; Gauss et al., 1998; Macri et al., 2012). Before defining the structure of HCNs, the evidence that, at variance with $\mathrm{K}$ channels, large cations such as $\mathrm{Ba}^{2+}$ or tetraethylammonium do not block $I_{\mathrm{h}}$ (DiFrancesco, 1981a; Ludwig et al., 1998) and 


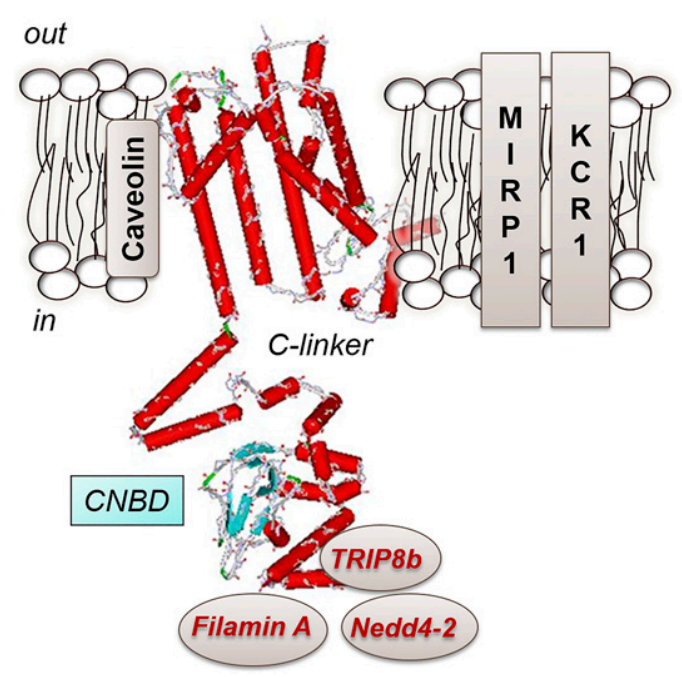

Fig. 1. HCN channel topology and associated interacting proteins. Transmembrane and cytoplasmic arrangement of the HCN1 channel subunit (Protein Data Bank code: $5 \mathrm{u} 6 \mathrm{p}$ ) with distinct domains for interaction with ancillary proteins predicted at the $\mathrm{N}$ terminus (caveolin) (Barbuti et al., 2012) and C terminus (Trip8b) (Saponaro et al., 2014); Filamin-A (Gravante et al., 2004); and Nedd4-2 (Wilkars et al., 2014). MIRP-1 (Yu et al., 2001) and KCR1 (Michels et al., 2008) interact also with HCN channels.

that substitution of Thr/Ser residue typical of $\mathrm{K}_{\mathrm{v}}$ channels (Zhou and MacKinnon, 2004) with Cys peculiar of HCN has no effect on selectivity (Macri et al., 2012), led to hypothesize the existence of a functionally wider pore. The recent definition of HCN (Lee and MacKinnon, 2017) provides an intriguing explanation: as stated, only two filter sites are present in the HCN pore, at variance with the four sites of $\mathrm{K}_{\mathrm{v}}$ channels; moreover, surrounding amino acids reorientate filter amino acids, namely Tyr. Selectivity depends on the limited space allowed by two $\mathrm{K}^{+}$ions, aligned and bound to the filters in $\mathrm{K}_{\mathrm{v}}$ channels. In the presence of a singlebound $\mathrm{K}^{+}$in $\mathrm{HCN}, \mathrm{Na}^{+}$ions can easily permeate the pore, without binding, thus conducting inward current (Lee and MacKinnon, 2017), with an exceptionally low ( $\sim 1$ picoSiemens) unitary channel conductance $(0.5-$ 1.7 picoSiemens for $\mathrm{HCN} 1$ and $\mathrm{HCN} 2$, respectively) (DiFrancesco, 1986, Thon et al., 2013; Liu et al., 2016). These properties are essential for the functional role of $I_{\mathrm{h}}$. In fact, a net inward current flows during the diastolic depolarization of cardiac pacemaker cells ( $\sim-60 \mathrm{mV}$ ) resulting from the following: 1 ) the relative (opposite) contribution of $\mathrm{Na}^{+}$entry and $\mathrm{K}^{+}$exit through the open channels, moving along their electrochemical gradients, and 2) the relative permeability, approximately 4:1 for $\mathrm{K}^{+}$over $\mathrm{Na}^{+}$(McCormick and Pape 1990b; Ho et al., 1994; Ludwig et al., 1998; Santoro et al., 1998). External $\mathrm{K}^{+}$concentration greatly influences channel conductance (DiFrancesco, 1982; Maccaferri et al., 1993; Cerbai et al., 1994), i.e., elevation of extracellular $\mathrm{K}^{+}$due to repetitive firing can amplify $I_{\mathrm{h}}$ and promote depolarization.

Second, HCNs possess a standard voltage sensor in S4, very similar to depolarization-activated channels, i.e., with a regular sequence of positive charged amino acids (Lys and Arg) (Kaupp and Seifert, 2001). A possible explanation of the reverse voltage dependence of these channels comes from the recent structural study by Lee and MacKinnon (2017) on HCN1. They hypothesize that the extraordinary size of S4 and its closeness to the S5-S6 pore, combined with the packed conformation of the S5-S6 helices, compress the pore in a closed state when the membrane is depolarized. Eventually, the inward movement of S4 caused by hyperpolarization displaces the link between $\mathrm{S} 4$ and S5, pulling S5 far from S6 and opening the pore like a zipper, instead of closing it as in $\mathrm{K}_{\mathrm{v}}$ channels (Männikkö et al., 2002). Such a molecular coupling mechanism between S4, S5, and the C-linker [the region between S6 and the cyclic nucleotide binding domain (CNBD)] was inferred previously on the basis of pioneer studies on gating properties of sea urchin sperm flagellar HCN (SpHCN) by introducing cysteine in the S4-S5 and C-linker and using Cys cross-linking agents such as $\mathrm{Cd}^{2+}$ (Prole and Yellen, 2006).

Third, HCNs possess a distinctive, highly conserved region for cyclic nucleotide binding at the $\mathrm{C}$ terminus (CNBD) (Kaupp and Seifert, 2001). A detailed description of CNBD structure and structure-activity relationships for interaction with cyclic nucleotides is given in the section Modulation by Cyclic Nucleotides. The first insight of modulation by direct cAMP binding (and unbinding) as the primary mechanism of autonomic modulation came from the pioneer work of Dario DiFrancesco on $f$-channels (DiFrancesco and Tortora, 1991). Binding of cAMP is not required to open HCN channels, at variance with retinal and olfactory cyclic nucleotide-gated (CNG) channels (Kaupp and Seifert, 2002; Craven and Zagotta, 2006). However, cAMP promotes channel opening by increasing the open state probability (Thon et al., 2013), accelerating activation, and slowing deactivation (Wicks et al., 2011), thus ultimately modifying voltage dependence and activation kinetics. The CNBD acts as an autoinhibitory mechanism, with cAMP relieving inhibition (Viscomi et al., 2001; Wainger et al., 2001; Wang et al., 2002; Akimoto et al., 2014); it is also implicated, although via a different binding site, in the inhibitory effect of the regulatory subunit tetratricopeptide repeat-containing Rab8b-interacting protein (TRIP8b) of HCN in neurons (see section The Role of Ancillary Subunit and Regulatory Proteins) (Saponaro et al., 2014). Finally, the C-terminal intracellular region of HCN4 controlsupon cAMP binding - a conformational change, leading to the formation of a tetrameric gating ring (Zagotta et al., 2003; VanSchouwen et al., 2015).

\section{B. Biophysical Features of HCN Isoforms}

In patch-clamped cells, upon hyperpolarization below a threshold of -40 to $-60 \mathrm{mV}$, HCN channels activate in a time- and voltage-dependent manner, generating 
an inward current that does not inactivate (Fig. 2A). Driven by their respective electrochemical gradients, $\mathrm{Na}^{+}$and $\mathrm{K}^{+}$flow through the open channels as long as the hyperpolarizing step is maintained, generating a net inward current. Without recapitulating all biophysical features, which have been extensively described elsewhere (Biel et al., 2009), it is anyway necessary to highlight some $I_{\mathrm{h}}$ properties to understand the mechanisms underlying its modulation by exogenous and endogenous substances and the consequences of gain or loss of function in inherited and acquired diseases.

Heterologous re-expression of single isoforms, generating homomeric tetramers, demonstrates that HCNs possess different kinetics and voltage-dependent properties. Channel opening upon hyperpolarization generates a time-dependent inward current that can be interpolated by a single- or double-exponential function (see, for example, Wicks et al., 2011; Zong et al., 2012; Kim and Holt, 2013; Nakamura et al., 2013, and, for review of previous literature, Biel et al., 2009). The time constant (tau) of activation differs among isoform subtypes. In native tissue, it depends on the coassembling of different isoforms in the tetrameric channel, the presence of ancillary subunits, post-translational modifications including (de)phosphorylation and $N$-glycosylation, the effect of ligands, and, finally, experimental conditions such as ionic composition of extracellular milieu (Chen et al., 2001b; Xue et al., 2002; Altomare et al., 2001; Much et al., 2003; Whitaker et al., 2007; Ye and Nerbonne, 2009). When heterologously re-expressed, HCN1 homotetrameric channels exhibit the fastest kinetics of activation and HCN4 the slowest, and the two other isoforms are in between (Ludwig et al., 1999; Seifert et al., 1999; Stieber et al., 2003b; Stieber et al., 2005). Activation kinetics is strongly dependent on voltage: the more negative the step, the faster the activation. This is evident in Fig. 2A, in which a family of HCN1, HCN2, and HCN4 current tracings are plotted as a function of time. The second striking difference relates to the voltage dependence: when the relative amplitude is plotted against the voltage step, HCN1 exhibits the less negative threshold and voltage of half-maximal activation $\left(\mathrm{V}_{1 / 2}\right)$ and HCN2 the most negative (Ludwig et al., 1999; Seifert et al., 1999; Stieber et al., 2003b, 2005). Finally, voltage dependence and kinetics of activation of HCN2 and HCN4 are very sensitive to cAMP, at variance with $\mathrm{HCN} 1$ (Moroni et al., 2000; Zagotta et al., 2003).

In recombinant systems or native cells, the slow exponential activation of $I_{\mathrm{h}}$ is preceded by a small initial, instantaneous current. The molecular nature of this current is still questioned because variable biophysical and pharmacological properties (e.g., amplitude, sensitivity to $\mathrm{Cs}^{+}$, or organic blockers) have been reported, depending on the frequency of hyperpolarizing pulses, intracellular cAMP or $\mathrm{Cl}^{-}$concentrations, expression of ancillary subunits, and other experimental conditions
A
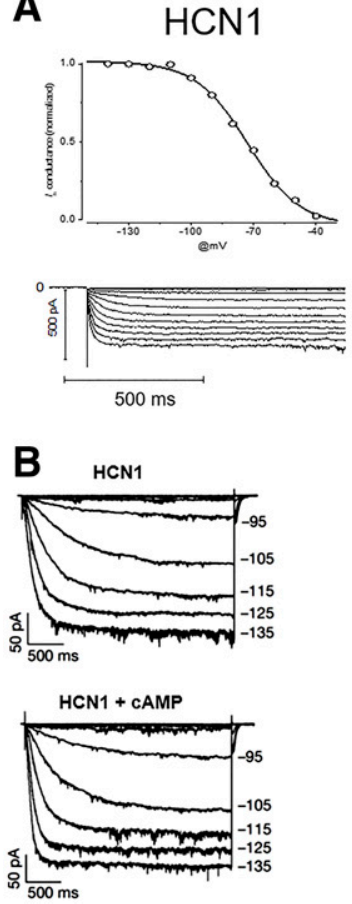

$\mathrm{HCN} 2$
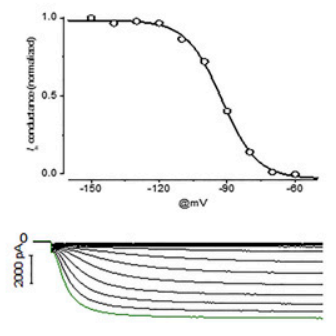

C

$\mathrm{HCN}$

$\mathrm{HCN} 2$

$\mathrm{HCN} 3$

$\mathrm{HCN} 4$
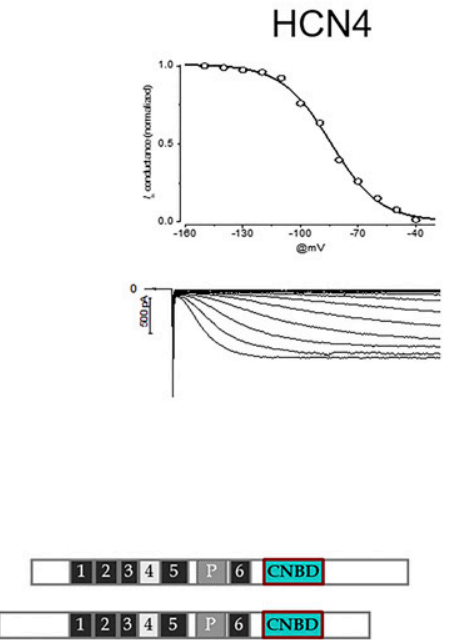

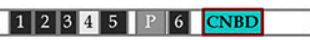

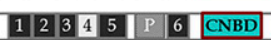

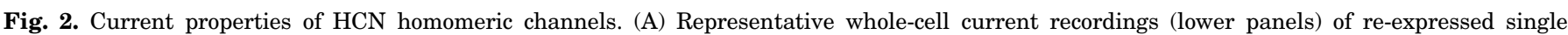

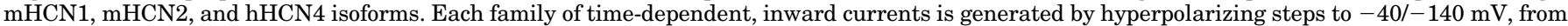

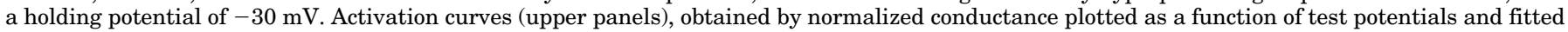

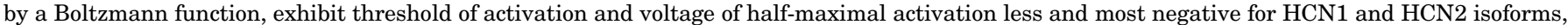

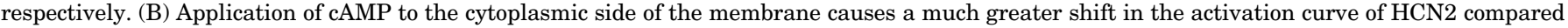
with HCN1 (modified from Wainger et al., 2001 with permission); (C) schematic representation of the structure of the four HCN isoforms. 
(Proenza et al., 2002; Mistrik et al., 2006; Proenza and Yellen, 2006). Recent results obtained with mutant HCN1 and HCN2 isoforms in the so-called zipper residues suggest that the two components (instantaneous and slow-activating current) may result from different open states of HCN channels (Wemhoner et al., 2012). At variance with the voltage- and time-dependent HCN current, the physiologic significance of the instantaneous current is unclear and may be dependent on cell types; indeed, a large voltage-independent inward current, blocked by $\mathrm{Cs}^{+}$and 4-(N-ethyl-N-phenylamino)-1,2 dimethyl-6-(methylamino) pyrimidinium chloride (ZD7288; see Pharmacology, section Ivabradine, Cilobradine, and Other Specific Bradycardic Agents), seems to contribute to neuronal excitability in stellate cells of ventral cochlear nucleus (Rodrigues and Oertel, 2006).

Several models have been proposed in the years to explain HCN behavior, namely voltage dependence, kinetics, and sensitivity to cAMP. Although those basic properties are well described by classic allosteric models (DiFrancesco, 1999), others are not. A more recent four-state model, in which HCN channels transit between two modes, tried to encompass this limitation and explain hysteresis, i.e., the voltage dependence of the HCN channels on their prior activity. In fact, the current-voltage activation curve of $I_{\mathrm{h}}$ is shifted positively after long hyperpolarizing steps (persistent channel opening), and shifted negatively after long depolarizations (closed channels) (Männikkö et al., 2005). The same authors suggest that transition between the two modes, or hysteresis, has important consequences for physiologic (and pathologic) functioning of rhythmic cells. In SAN cells, hysteresis keeps channels closed after an action potential (AP) until complete repolarization (and calcium current recovery), preventing a premature diastolic depolarization; attainment of maximum hyperpolarization (between two APs) stabilizes the open state of HCN channels, pushing the membrane potential toward the threshold for the next AP (Männikkö et al., 2005).

\section{Modulation by Cyclic Nucleotides}

1. Binding of $c A M P$ and Modification of Gating Properties. HCN channels are primarily activated by hyperpolarization of membrane potential and are regulated by cyclic nucleotides, which interact with a specific site, the CNBD, located in the intracellular C-terminal portion (see section HCN Modulation by Cyclic Nucleotides). cAMP accelerates channel opening and shifts the activation curve to more positive potential (DiFrancesco and Tortora, 1991). The shift in $\mathrm{V}_{1 / 2}$ depends on the channel subtype and conditions: it varies between 10 and $25 \mathrm{mV}$ for HCN2 and HCN4 (see Wahl-Schott and Biel, 2009 and references cited therein), but only $2-6 \mathrm{mV}$ for HCN1 (Wainger et al., 2001; Wang et al., 2001) (Fig. 2B). cAMP potency ( $\mathrm{EC}_{50}$, i.e., the concentration of ligand that produces a half- maximal voltage shift) is in the range $0.06-1.53 \mu \mathrm{M}$ (Table 1).

According to a generally accepted model, the channel is normally inhibited and the binding of cAMP removes this inhibition, inducing conformational changes and increasing the open probability of channel pore (Wainger et al., 2001). As suggested by Wang et al. (2001) from experiments involving HCN1-HCN2 chimera, the different sensitivity of these two isoforms depends on differences in the sequences involved in the interactions between CNBD and the C-linker. Studies performed on the isolated C-terminal domain (C-linker + CNBD) of HCN2 showed that addition of cAMP changes the proportion between monomer and tetramer in favor of the latter (Zagotta et al., 2003). There is evidence that the isolated $\mathrm{C}$-terminal domain of $\mathrm{HCN} 1$ has a higher propensity to tetramerize than those of $\mathrm{HCN} 2$ and HCN4 (Lolicato et al., 2011; Chow et al., 2012). Thus, a possible explanation to the lower sensitivity of HCN1 to the natural agonist could be a sort of preactivation of the channel (Chow et al., 2012), probably because it is able to trap endogenous cAMP in the CNBD (Lolicato et al., 2011). The positive effect of cAMP on tetramerization of the isolated intracellular region is translated, in the whole channel, in conformational changes that propagate from the CNBD via the C-linker to the $\mathrm{S} 6$ fragment (Lee and MacKinnon, 2017).

Surprisingly, HCN3 is not activated by cAMP; rather, the activation curve is slightly shifted to more negative voltages (Mistrík et al., 2005; Stieber et al., 2005). The lack of sensitivity of HCN3 to cAMP, despite the presence of a functional CNBD in the intracellular region, has been explained by a shorter C-terminal sequence, after the CNBD, which alters the normal autoinhibition of the channel (Stieber et al., 2005).

In recent years, several biophysical techniques, such as isothermal titration calorimetry, surface plasmon resonance, and fluorescence anisotropy, have allowed measurement of the binding affinity of cAMP for the CNBD. Lolicato et al. (2011) used surface plasmon resonance to compare the interaction of cAMP with the C-terminal domain of $\mathrm{HCN} 1,2$, and 4, finding that the three isoforms recognize the natural agonist with similar $\mathrm{K}_{\mathrm{D}}$ values (respectively, 5,10 , and $11 \mu \mathrm{M}$ ). $\mathrm{Xu}$ et al. (2010) used isothermal titration calorimetry and fluorescence anisotropy to measure affinity on the intracellular portion of $\mathrm{HCN} 2$ and 4, comparing the results with the $\mathrm{EC}_{50}$ (Table 1); because human HCN4 (hHCN4) does not express in Xenopus laevis oocytes, they used a murine HCN2 (mHCN2)-hHCN4 chimera, in which the C-linker and CNBD of $\mathrm{HCN} 2$ were replaced by the same portion of HCN4. cAMP potency, measured in functional studies on the whole channel, was three times higher on HCN2 than on HCN4, but the same difference was not found for affinity (measured on the purified cytosolic domains), giving evidence that binding and gating efficacy might have different requirements. 
TABLE 1

Potency of cyclic nucleotides on different HCN channel isoforms

$\mathrm{EC}_{50}$ is the concentration of ligand that produces a half-maximal voltage shift.

\begin{tabular}{lcll}
\hline Cyclic nucleotide & $\mathrm{EC}_{50}(\mu M)$ & \multicolumn{1}{c}{ Isoform/Tissue } & \multicolumn{1}{c}{ References } \\
\hline cAMP $^{a}$ & 0.06 & mHCN1 (Xenopus laevis) & Wang et al. (2001) \\
& 0.10 & mHCN2 (Xenopus laevis) & Wang et al. (2001) \\
& 0.50 & mHCN2 (HEK293 cells) & Ludwig et al. (1998) \\
& 0.80 & mHCN2 (Xenopus laevis) & Zagotta et al. (2003) \\
& 1.0 & mHCN2 (HEK293 cells) & Zong et al. (2012) \\
& 0.08 & mHCN2 (Xenopus laevis) & Xu et al. (2010) \\
& 0.24 & mHCN2-h4 chimera (Xenopus laevis) & Xu et al. (2010) \\
& 1.53 & hHCN4 (HEK293 cells) & Milanesi et al. (2006) \\
& 0.21 & Rabbit SAN myocyte & DiFrancesco and Tortora (1991) \\
cGMP & 0.72 & SpHCN (sea urchin sperm) & Kaupp and Seifert (2001) \\
& $6.0^{a}$ & mHCN2 (HEK293 cells) & Ludwig et al. (1998) \\
& $8.3^{a}$ & mHCN2 (Xenopus laevis) & Zagotta et al. (2003) \\
& $13.2^{a}$ & hHCN4 (HEK293 cells) & Lolicato et al. (2014) \\
& $7.85^{a}$ & Rabbit SAN myocyte & DiFrancesco and Tortora (1991) \\
cCMP & $479^{b}$ & SpHCN (sea urchin sperm) & Kaupp and Seifert (2001) \\
& 29.0 & mHCN2 (HEK293 cells) & Zong et al. (2012) \\
& 11.85 & Rabbit SAN myocyte & DiFrancesco and Tortora (1991) \\
\hline
\end{tabular}

${ }^{a}$ Full activator.

${ }^{b}$ Partial activator.

2. Other Cyclic Nucleotides. As well as cAMP, guanosine- $3^{\prime}, 5^{\prime}$-cyclic monophosphate (cGMP) is a full agonist of HCN channels: at saturating concentrations, the shift in $\mathrm{V}_{1 / 2}$ is in the same range as that of cAMP. However, cGMP displays a 10 -fold lower potency for HCN channels (Table 1) (Ludwig et al., 1998). cGMP and cAMP bind to the CNBD in a similar way, only differing in the orientation of the purine ring (Zagotta et al., 2003). On SpHCN, cGMP behaves as a partial agonist showing, when compared with cAMP, an intrinsic activity of 0.5 and about 600 -fold lower affinity (Kaupp and Seifert, 2001).

Cytidine $3^{\prime}, 5^{\prime}$-cyclic monophosphate (cCMP) is also able to modulate HCN channels, behaving as partial agonist (DiFrancesco and Tortora, 1991). On HCN2 and HCN4 channels, cCMP shifts the activation curve to more positive potentials, speeds up current activation, and decreases current deactivation, but it has no effect on HCN1 and HCN3. The voltage shift and the maximal increase in the current amplitude were significantly smaller than those observed for cAMP, in agreement with the behavior of a partial agonist (Zong et al., 2012). On HCN2, uridine $3^{\prime}, 5^{\prime}$-cyclic monophosphate (cUMP), purine $3^{\prime}, 5^{\prime}$-cyclic monophosphate (cPMP), and 2-amino-cPMP are all able to shift the activation curve to more positive potentials and to increase channel conductance, with efficacy similar to cAMP and cGMP; on the contrary, inosine $3^{\prime}, 5^{\prime}$-cyclic monophosphate (cIMP), as well as cCMP, behaves as weak activators ( $\mathrm{Ng}$ et al., 2016).

In addition to cyclic mononucleotides, also cyclic dinucleotides can modulate HCN channels. In mouse SAN myocytes, they behave as antagonists, being able to reduce $I_{\mathrm{h}}$ (Lolicato et al., 2014). Cyclic [guanosine-( $2^{\prime}-$ $\left.5^{\prime}\right)$-monophosphate-adenosine-( $\left.3^{\prime}-5^{\prime}\right)$-monophosphate], the cyclic dinucleotide that has been found in mammals, caused a shift of the activation curve toward more negative potentials, and a $\sim 30 \%$ reduction of firing rate. On HCN4 channel expressed in human embryonic kidney (HEK)293 cells, the dose-response curve yielded an $\mathrm{IC}_{50}$ (i.e., the concentration of ligand that produces a half-maximal voltage backshift) of $114 \mathrm{nM}$. Other cyclic dinucleotides could completely reverse the effect of cAMP on the activation curve, as, for instance, cyclic di- $\left(3^{\prime}, 5^{\prime}\right)$-GMP, which was $\sim 16$ times less potent than cyclic [guanosine- $\left(2^{\prime}-5^{\prime}\right)$-monophosphate-adenosine- $\left(3^{\prime}-5^{\prime}\right)$ monophosphate] ( $\left.\mathrm{IC}_{50} 1.8 \mu \mathrm{M}\right)$.

\section{The Role of Ancillary Subunits and Regulatory Proteins}

Different regulatory proteins form macromolecular complexes with HCN channel subunits and define the features of HCN-mediated current in vivo, the regional or subcellular localization of HCN proteins, as well as their susceptibility to modulatory signals.

The number of proteins acting as HCN-binding partners has grown over the last decades; for some proteins, such as Mint2 (Mun18-interacting protein), synaptic scaffolding molecule, and tamalin, only a scaffold function for HCN2 has been identified (Kimura et al., 2004), and a possible role in channel trafficking, distribution, and clustering has been hypothesized.

MinK-related peptide 1 (MiRP1), encoded by kcne2 gene, is a single-transmembrane domain protein, which serves as regulatory subunit of different cardiac ion channels, including HCN channels. It enhances HCN protein and current expression in an isoform-specific manner (Yu et al., 2001; Decher et al., 2003; Qu et al., 2004; Brandt et al., 2009). When coexpressed with HCN channel, current kinetics of activation is accelerated for HCN1 and HCN2, whereas it is slowed down for HCN4, which also displays a shift of the midpoint of activation to more negative voltages. High levels of MiRP1 and HCN subunits (primarily HCN4, and, depending on the 
species, HCN1 or HCN2) are expressed in the SAN of small and large mammals, including mouse, rabbit, and human (Yu et al., 2001; Accili et al., 2002; Schweizer et al., 2009). A similar interaction among MiRP1 and HCN4, 2, and 1 most likely occurs in atrial and ventricular myocytes, where transcript levels of both subunits are lower compared with SAN (Yu et al., 2001; Decher et al., 2003; Qu et al., 2004; Stillitano et al., 2008, 2013; Sartiani et al., 2010). In these regions, the modification of HCN-mediated current-related to cardiac diseases [atrial fibrillation (AF) and ventricular hypertrophy] or to postnatal maturation-is most likely associated with transcriptional regulation of both HCN channel and MiRP1 subunit.

The $\mathrm{K}^{+}$channel regulator 1 (KCR1) is a transmembrane protein expressed in cerebellum and heart (Hoshi et al., 1998; Michels et al., 2008). Protein expression analysis in rat and guinea pig revealed an extensive distribution of the protein in the heart with larger amount in the atrioventricular node (AVN), left atrium, and ventricle, followed by right atrium and ventricle, and SAN. KCR1 is a regulatory subunit of diverse native $\mathrm{K}_{\mathrm{v}}$ channels, as well as HCN channels. In heterologous expression systems and native ventricular myocytes, KCR1 interacts with $\mathrm{HCN} 2$, reducing current size and shifting $I_{\mathrm{h}}$ activation to more negative potentials. These modifications ultimately decrease spontaneous rhythmicity in cultured neonatal cardiac myocytes, suggesting that KCR1 may be an important regulatory subunit of HCN current in vivo.

Caveolin-3 is a lipid raft component of myocyte membrane that colocalizes with and affects the expression and function of HCN channels, as well as their susceptibility to modulating signals. In SAN cells, interaction between caveolin-3 and HCN4 affects channel voltage dependence by shifting $\mathrm{V}_{1 / 2}$ to negative voltages; it also modifies HCN4 current kinetics by accelerating channel deactivation (Barbuti et al., 2004, 2007,2012 ). Because $\beta_{2}$-adrenoceptors, but not $\beta_{1}$, localize to lipid rafts in the SAN, their activation generates a prominent signal mediating the adrenergic enhancement of HCN current and the rise of heart rate (Barbuti et al., 2007). Following investigations demonstrated that a similar colocalization between caveolin-3 and HCN4 channels occurs in human atrial and embryonic stem cell-derived cardiomyocytes (Bosman et al., 2013; Stillitano et al., 2013). In the latter, the shift of $\mathrm{V}_{1 / 2}$ is directly related to the increase of caveolin-3 expression during myocyte maturation.

Filamin A is a cytosolic scaffolding protein that exerts a crucial role for the trafficking of numerous ion channels in excitable cells, including neurons and cardiac myocytes. It anchors ion channels to actin cytoskeleton and clusters them in distinct locations on cell surface membrane. In the brain, only HCN1, but not HCN2, 3, or 4, associates with filamin A (Gravante et al., 2004). In heterologous expression systems, this interaction reduces the density of channel expression as well as whole-cell conductance by aggregating HCN1 within restricted regions of the cell membrane. Additionally, filamin A promotes a reversible dynamindependent internalization of HCN1 channels and a redistribution of HCN1 channels on cell surface by accumulation of channels in endosomal compartments (Noam et al., 2014). In cultured hippocampal neurons, expression of a dominant-negative filamin A increases the expression of native HCN1, whereas acute abrogation of HCN1-filamin A interaction enhances current size. Whether a similar interaction occurs at cardiac level is unknown, despite filamin A being present in mouse and human atrial myocytes (Rafizadeh et al., 2014).

$T R I P 8 b$, also termed Pex5p-related protein (PEX5Rp), and H-channel interacting protein 1 (HIP1), is a brain cytoplasmic protein, member of the Rab family of small GTPase proteins, which are important for vesicle trafficking (Chen et al., 2001a). In neocortical and hippocampal pyramidal neurons, colocalization of TRIP8b to HCN1 promotes an active trafficking of the channels from soma to dendrites that is important to modulate spike firing and synaptic potential (Santoro et al., 2004; Zolles et al., 2009). In TRIP8b-knockout (KO) mice, HCN surface expression in hippocampal pyramidal neurons is dramatically reduced, as well as current size in this region; moreover, normal expression pattern of $\mathrm{HCN}$ channels is profoundly altered in pyramidal neuron dendrites (Lewis et al., 2011). Nine isoforms of TRIP8b have been identified that differentially affect HCN channel gating, membrane expression, and trafficking in the nervous system. The overexpression of TRIP8b in cultured hippocampal pyramidal neurons or heterologous expression systems exerts different effects on HCN1 surface expression according to the variant type. TRIP8b (1a-4) and TRIP8b (1a), major splice variants in the hippocampus, enable a correct localization of HCN1 (Lewis et al., 2009; Santoro et al., 2009). Moreover, TRIP8b (1a-4) upregulates HCN1 expression in heterologous systems and promotes its dendritic expression. Conversely, TRIP8b (1a) downregulates HCN1 surface expression in $X$. laevis oocytes and inhibits the abnormal expression of HCN1 in the axons of pyramidal neurons (Piskorowski et al., 2011). Opposed to TRIP8b, a recent paper shows that ubiquitination by the Nedd 4-2 (neuronal precursor cell-expressed developmentally downregulated four-like) protein decreases $\mathrm{HCN} 1$ surface expression and translocation, leading to $I_{\mathrm{h}}$ loss of function (Wilkars et al., 2014).

\section{E. From Transcriptional Control to Post-Translational Modifications}

1. microRNAs. Accumulating evidence on sinus node dysfunctions has led to consider the downregulation of HCN channels as possible common cause of different conditions leading to bradycardia (D'Souza 
et al., 2015). The role of microRNAs (MiR) is emerging as main transcriptional regulator of $\mathrm{HCN}$ channels in cardiac pacemaker centers. MiR-1, one of the main muscle-specific microRNA (myomiR), is induced by athletic training in the heart jointly to a downregulation of the transcription factors Tbx3 and NRSF (D'Souza et al., 2014). These changes are consistent with the downregulation of HCN 4 and $I_{\mathrm{h}}$ found in SAN cells of trained animals.

On the contrary, in different cardiac pathologies, including myocardial infarction (Suffredini et al., 2012; $\mathrm{Yu}$ et al., 2015) or age-related $\mathrm{AF}$ (Li et al., 2015b), expression of HCN channels is upregulated, in line with the observed reduction of MiR-1 levels detected in the ventricles and atria. Accordingly, in rats with myocardial infarction, administration of ivabradine, a selective bradycardic agent (Suffredini et al., 2012), or spironolactone, an aldosterone blocker ( $\mathrm{Yu}$ et al., 2015), counterbalances the overexpression of HCN channels in parallel with upregulation of MiR-1.

In all conditions, the mechanism responsible for the modifications of microRNA levels in the sinus node or in the working myocardium remains unknown.

2. Regulation by Membrane Phosphoinositides. Signaling pathways coupled to membrane phosphoinositide content and downstream derivatives stimulate HCN channels.

Phosphatidylinositol 4,5-bisphosphate [ $\mathrm{PI}(4,5) \mathrm{P} 2]$ is a membrane constitutive component that increases the opening of recombinant and native HCN channels by shifting the voltage dependence of activation to more positive potentials by $5-20 \mathrm{mV}$, depending on isoforms. It acts as intracellular allosteric activator that facilitates channel opening (Biel et al., 2009).

In the heart, basal variability of membrane PI $(4,5) \mathrm{P} 2$ is likely to contribute to the variations in the voltage dependence of $I_{\mathrm{h}}$ activation in cardiac cells at different maturation degrees (Cerbai et al., 1999b; Qu et al., 2001), derived from different regions, or following stress and pathologic conditions (Suh and Hille, 2007).

In SAN cells, stimulation of bradykinin $\mathrm{BK}_{2}$ receptors coupled to phospholipase $\mathrm{C}$ enhances phosphatidylinositol kinase activity that in turn stimulates polyphosphoinositide synthesis, thereby enhancing HCN channel function (Pian et al., 2007). A similar interaction with phosphoinositides occurs in neurons, where $\mathrm{PI}(4,5)$ $\mathrm{P} 2$ acts as an allosteric modulator of $\mathrm{HCN}$, causing a rightward shift of voltage activation (Zolles et al., 2006; Ying et al., 2011); gating by phosphoinositides may be important to maintain rhythmogenesis when signaling pathways leading to phospholipid degradation reduce channel activation in nerve cells. Recent data in SpHCN suggest that $\mathrm{PI}(4,5) \mathrm{P} 2$ binds to both the transmembrane core region and the C-linker domain of the channel, with opposite effects (Flynn and Zagotta, 2011). Whether such a dual mechanism also occurs in mammalian $\mathrm{HCN}$ is unknown.
Other allosteric modulators of HCN channels are two membrane phosphoinositide derivatives, phosphatidic acid and arachidonic acid, which are products of diacylglycerol kinase and phospholipase A2, respectively. They directly facilitate HCN channel gating by shifting the voltage dependence of activation to more positive values (Fogle et al., 2007).

3. Kinases. Phosphorylation status of HCN channels is an additional regulatory mechanism controlling HCN properties and adapting its activity to the peculiar conditions of different types of cardiac cells and neurons.

Despite the fact that HCN channels are considered end effectors of cAMP, experimental evidence demonstrated a modulatory role of protein kinase A (PKA). In early cardiomyogenesis, activation of PKA has an exclusive role in the stimulation of $\mathrm{HCN}$ current following $\beta$-adrenergic receptor stimulation (AbiGerges et al., 2000). In adult mouse SAN, PKA seems to exert an additive positive shift of the activation curve upon $\beta$-adrenergic receptor stimulation (Liao et al., 2010), although the mechanism is most likely mediated by PKA-dependent effects on cAMP production or diffusion rather than phosphorylation of the channel (St Clair et al., 2013).

In hippocampal neurons, phospholipase C-protein kinase $\mathrm{C}$ activation, phosphorylating $\mathrm{HCN} 1$ isoform, decreases HCN current and HCN1 surface expression (Williams et al., 2015). Similar findings were obtained in respiratory neurons within the pre-Bötzinger complex (Thoby-Brisson et al., 2003).

Tyrosine kinases of the Src family have also a stimulatory function in the mature cardiac cells as well as in different types of neurons (Santoro et al., 1997; Wu and Cohen, 1997; Yu et al., 2004; Zong et al., 2005; Arinsburg et al., 2006). In these cells, the pathway contributes to regulate spontaneous electrogenesis by direct phosphorylation of HCN1, HCN2, and HCN4 and speeding of channel kinetics. Despite the fact that the residue (Y476) conferring such modulation in HCN2 channels is conserved in the other isoforms, a stimulation of kinetics by Src tyrosine kinase has been proven only for HCN4. In addition, the latter isoform undergoes also a positive shift $(\cong+10 \mathrm{mV})$ of voltage dependence, most likely because of an additional phosphorylation in a different tyrosine residue (Y531) ( $\mathrm{Li}$ et al., 2008a). In rat ventricular myocytes, the receptor-like protein-tyrosine phosphatase- $\alpha$ controls the extent of HCN2 channel phosphorylation in this site, shifts the voltage dependence of activation, and decreases channel insertion into the membrane (Huang et al., 2008).

In neuronal cells, HCN channels are also phosphorylated by the serine/threonine kinase, p38 mitogenactivated protein kinase (Poolos et al., 2006), and calcium/calmodulin-dependent protein kinase II (Shin and Chetkovich, 2007). 
Modulation by the small ubiquitin-like modifier (SUMO) peptide is one of the mechanisms involved in the regulation of protein-protein interactions. HCN2 SUMOylation occurs in mouse forebrain tissue (Parker et al., 2017); in transfected HEK cells, SUMOylation of HCN2 increased current conductance and surface expression. This finding is of interest in view of $\mathrm{HCN}$ dysregulation in central nervous system (CNS) diseases, as discussed later.

\section{Origins, Physiology, and Pathophysiology of HCN Channels}

\section{A. HCN: Ancient and Early Channels}

1. Phylogeny. HCN channels belong to the superfamily of six-transmembrane segment channels and are related to CNG channels and voltage-dependent ether-a-go-go $\mathrm{K}^{+}$channels $\mathrm{K}_{\mathrm{v}} 10-\mathrm{K}_{\mathrm{v}} 12$ (Lee and MacKinnon, 2017). Being components of the ancestral gene pattern, HCN channels are present across a wide spectrum of invertebrate and vertebrate species. They most likely derive from a single ancestral gene subjected to duplications and diversification events over the species that eventually generated four different isoforms prior to the origin of the vertebrate clade (Jackson et al., 2007). A functional role for HCN in setting heart rhythm has been postulated on the basis of pharmacological studies also in vertebrate ancestors (Wilson and Farrell, 2013). Based on sequence conservation analysis, HCN3, thought to be most similar to the ancestral channel, was the first to diverge as a product of the first duplication. HCN3 was followed by the emergence of HCN4 and then of HCN1 and HCN2, thus composing a group of four variants that collectively shares $80 \%-90 \%$ sequence conservation within the core and transmembrane regions. The latter in all vertebrate and invertebrate contribute to common functional properties of $\mathrm{HCN}$ channels. The residual variations in these regions are responsible for subtle isoform-specific differences related to inner selectivity filter, rates of channel opening, and differences in cAMP efficacy to modulate $\mathrm{HCN}$ isoforms.

In all four vertebrate isoforms, a region of $\sim 50$ residues upstream to the start of $\mathrm{S} 1$ in the $\mathrm{NH}_{2}$ terminus is conserved. In mouse $\mathrm{HCN} 2$, this region is involved in intersubunit interactions of tetramer assembly and in the formation of functional channels (Tran et al., 2002). Analogous regions present in the other isoforms are supposed to exert similar functions.

In all vertebrate $\mathrm{HCN} 1,2$, and 4, but not in $\mathrm{HCN} 3$, a different block in the $\mathrm{COOH}$ terminus is conserved. It represents a PDZ-binding domain enabling channels to interact with PDZ-containing proteins and with the TRIP8b protein (Kimura et al., 2004; Santoro et al., 2004), which regulates channel surface expression.

2. HCN Channels in Stem Cells. The growing number of studies in different types of stem cells has led to identify the presence of several specialized ion channels, including HCN channels (Heubach et al., 2004; Wang et al., 2005; Sartiani et al., 2007). As suggested for other bioelectric signals, mainly $\mathrm{K}^{+}$or $\mathrm{Ca}^{2+}$ channels, HCN channels also may act as regulators of a wide range of stem cell functions, including proliferation, migration, differentiation, and tissue regeneration of nonexcitable cells (Blackiston et al., 2009; Sundelacruz et al., 2009, 2015; Levin, 2014). Indeed, HCN-mediated mechanisms are most likely involved in the development of a mature phenotype of olfactory sensory neurons, where HCN channels are expressed precociously and drive axon organization (Mobley et al., 2010).

Expression pattern of HCN channels in stem cells is species- and/or origin-dependent, despite the relatively homogenous phenotype of potency markers ( $\mathrm{Li}$ and Deng, 2011). HCN1 channels are highly expressed in human pluripotent stem cells, but not in mouse cells, where HCN3 largely predominates, suggesting that current phenotype and regulation might diverge among species, as observed in differentiated cells. Differently, human bone marrow-derived stem cells express HCN2 channels. Despite the presence of HCN transcripts and proteins in stem cells, only one study has been performed in mouse pluripotent stem cells, where the channels were found involved in cell proliferation, in particular in cell cycle progression from $G_{0}$ to $G_{1}$ phase (Lau et al., 2011).

\section{HCN during Organogenesis.}

a. Cardiogenesis. Occurrence of spontaneous electrical activity is an early event in cardiac morphogenesis. In the mouse, the whole process has been thoroughly dissected, identifying that this pacemaker activity is detectable since embryonic day (E) 7.5 in precardiac mesoderm (cardiac crescent) of the first heart field (Liang et al., 2013; Später et al., 2013; Barbuti and Robinson, 2015). Cells comprised in this early pacemaker region provide efficient peristaltic contractions necessary in the primitive heart tube and express two distinct markers, the transcription factor Nkx2-5 and HCN4 channels (Christoffels et al., 2010). Lineage-tracing experiments show that the $\mathrm{Nkx} 2-5^{+} /$ $\mathrm{HCN}^{+}$cells do not give rise to the cardiac conduction system in the developing heart, but generate atrial and ventricular precursors (Moorman and Christoffels, 2003). At E8, posterior heart field precursors expressing the transcription factor Tbx18 start to proliferate and will form the sinus venosus, a symmetric structure of the heart tube. Following expression of two additional markers (Shox2 and CD166), these cells will become the leading pacemaker (SAN precursors), when at E9.5 a subgroup of cells further expresses the second heart field marker Isl-1 and the transcription repressor Tbx3.

Subsequent increase of Tbx 3 and HCN4 and decrease of CD166 expression ultimately form the sinus atrial 
node in the right atrium, whose typical panel of markers will maintain Isl-1, Tbx18, Shox2, Tbx3, and a high level of HCN4 throughout life. In the left atria, activity of the homeobox factor Pitx2c specifically suppresses the SAN gene program, allowing the correct asymmetric development of the conduction system (Mommersteeg et al., 2007). Despite the fact that HCN4 is functionally expressed ever since the appearance of the pacemaker activity in the precardiac mesoderm, HCN4 starts to play a fundamental function during the formation of the SAN between E9.5 and E11.5, since global and cardiacspecific HCN4-KO mice die in this developmental stage (Stieber et al., 2003a). However, studies in HCN4deficient embryos show significantly reduced contraction rates and immature $\mathrm{AP}$ in deficient embryos compared with wild type (WT), indicating that even before SAN formation HCN4 is critical for normal cardiac development.

Studies with specific deletion of HCN1 and HCN2 confirmed the role of HCN4, because HCN1 (Nolan et al., 2003) or HCN2 (Ludwig et al., 2003; Cao and Oertel, 2011) KO animals do not evidence major cardiac alterations in developing embryos. This also suggests that expression of HCN1 and HCN2 channels in the heart is delayed during cardiogenesis. Indeed, transcription profiling throughout mouse cardiac development indicates that embryonic heart before E9.5 displays abundant HCN4 transcript, whereas other HCN transcripts are almost absent. Toward birth, HCN transcription profile in the atrium and ventricle changes remarkably, because HCN4 is strongly downregulated, whereas HCN1 and HCN2 transcripts slowly emerge. HCN3 isoform shows highest levels at early embryonic stages and then fades to very low levels (Schweizer et al., 2009).

Little information is available on the developmental changes of HCN channels occurring in humans. In a limited time window, i.e., from 11 to 14 weeks of fetal life, a strong protein expression of $\mathrm{HCN} 4$ is present in the whole heart that contributes to a remarkable HCNmediated current in ventricular myocytes (Bosman et al., 2013). At this stage, current density is larger than that retrieved in human healthy adult ventricular cardiomyocytes and close to the values described in ventricular myocytes from ischemic patients (Fig. 3), corroborating the concept of HCN channel expression as a marker of fetal gene reprogramming in cardiac disease (see Altered HCN function and cardiac disease).

b. Insights from stem cell-derived cardiomyocytes. A different approach to address the developmental changes during cardiogenesis consists in the use of pluripotent stem cells, whose cardiomyogenic potential in vitro is well established and widely used in the last decades. Following established protocol of differentiation, the in vitro model recapitulates many of the developmental stages described for in vivo cardiogenesis, thus representing a highly valuable tool to investigate the embryonic/fetal modifications of cardiac cells difficult to address in other models. It is also well known that the cardiomyocyte population arising from this model is heterogeneous and composed of atrial-, ventricular-, and SAN-like cells that have been thoroughly characterized for their electrical and structural properties more than 20 years ago (Maltsev et al., 1993). Molecular insights have been reviewed recently (Barbuti and Robinson, 2015). An interesting finding emerged from mouse pluripotent cells is the variable propensity to develop SAN-like or atrial/ventricularlike lineage. The former is characterized by a prominent expression of HCN4/HCN1 channels that are colocalized with caveolin-3 and present together with T-type calcium channels $\mathrm{Ca}_{\mathrm{v}} 3.1 / 3.2$, thus recapitulating the pattern of channels typically expressed in native SAN (van Kempen et al., 2003; Marionneau et al., 2005; Yanagi et al., 2007; Barbuti et al., 2009). Differently, atrial/ventricular-like lineage is characterized by higher levels of HCN2 and HCN3 compared with HCN4 and HCN1 (White and Claycomb, 2005; Qu et al., 2008).

Phenotype heterogeneity and lineage preference also feature human pluripotent stem cells (He et al., 2003; Mummery et al., 2003), where atrial/ventricular phenotypes associate with HCN2 preponderance over HCN4 (Satin et al., 2004). Consistency of findings among different mammalian species warrants further investigation to better understand whether distinct, still unidentified pluripotency states drive a preferential lineage specification. This hypothesis applies to recent studies on pacemaking activity in early (days 11-21 of differentiation) cardiomyocytes from pluripotent cells (Weisbrod et al., 2013), where HCN current, detectable in most of beating cells, is determined by HCN4 and HCN2 isoforms. The concomitant association with $\mathrm{Ca}_{\mathrm{v}} 1.3, \mathrm{Na}^{+} / \mathrm{Ca}^{2+}$ exchanger (NCX)-1, and Tbx3 in these cells defines a panel of genes typical of human SAN and perinodal areas (Chandler et al., 2009), suggesting that these cells are early pacemaker or SAN-like cells rather than immature atrial or ventricular cardiomyocytes. Accordingly, cell lines prone to develop atrial/ventricular phenotype exhibit properties resembling those present in adult atrial/ventricular cells, such as expression of $\mathrm{Na}_{\mathrm{v}} 1.5, \mathrm{Ca}_{\mathrm{v}} 1.2$, and HCN2, whereas $\mathrm{Ca}_{\mathrm{v}} 1.3$ and HCN4 are absent (Satin et al., 2004). Differently, studies from our group, performed in a different cell line maintained in long-term culture, evidence the early emergence of immature SAN-like cells, representing the most frequent cardiomyocyte population after 1530 days of differentiation. This single phenotype diverges into distinct atrial/ventricular myocytes at later maturation stages (55-110 days) (Sartiani et al., 2007; Paci et al., 2012; Bosman et al., 2013). During this developmental period in vitro, $\mathrm{HCN}$ channel expression shifts from a SAN-like pattern, mainly composed of $\mathrm{HCN} 1$ and $\mathrm{HCN} 4$, to an atrial/ventricular pattern, 


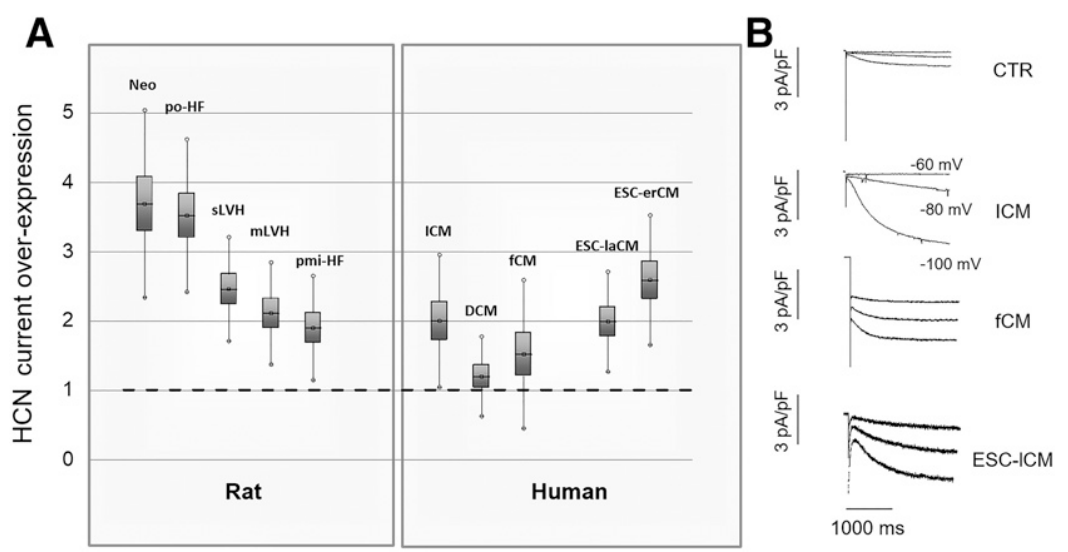

Fig. 3. $I_{\mathrm{h}}$ current expression in cardiomyopathies and in cardiac development. (A) Data points represent the ratio between current density measured in ventricular myocytes from diseased hearts and respective control. In neonatal rat cardiomyocytes (Neo), values measured at 2 weeks are compared with those at 2 days after birth. po-HF, pmi-HF: relative increase of $I_{\mathrm{h}}$ in rats with overt heart failure, resulting from pressure overload or following myocardial infarction, respectively. $\mathrm{mLVH}, \mathrm{sLVH}$ : relative increase of $I_{\mathrm{h}}$ in rats with mild or severe left ventricular hypertrophy caused by aortic banding or long-lasting pressure overload, respectively. DCM, ICM: relative increase of $I_{\mathrm{h}}$ in patients undergoing cardiac transplantation for terminal dilated or ischemic cardiomyopathy, respectively. For all conditions, the relative increase of current density is statistically significant versus controls, that is, normotensive rats, sham-operated rats, or undiseased donor hearts not transplanted for technical reasons, with the exception of DCM patients. fCM, ESC-laCM, and ESC-erCM: relative increase of $I_{\mathrm{h}}$ in human fetal (11-14 weeks) cardiomyocytes and in cardiomyocytes differentiated from human embryonic stem cells in late and early developmental stages, respectively. (B) Representative recordings of $I_{\mathrm{h}}$ in a control (CTR), a hypertrophic (ICM), a fetal (fCM), and a late ESC-derived cardiomyocyte (ESC-laCM).

where HCN2 isoform predominates and remains constant. The functional counterpart in the early stage is defined by a robust HCN current, which throughout maturation declines in amplitude and activates much slower, in accordance to a lower expression of HCN1, approaching values like those encountered in native human fetal and adult hypertrophic ventricular cardiomyocytes (Fig. 3). The findings further strengthen the similarities between in vitro and in vivo cardiac differentiation. The advantageous property has been further exploited to investigate the subcellular compartmentation of HCN4 channel in the human setting. During development, HCN4 protein signal shifts from a widespread localization in $\alpha$-actinin-positive immature cells to restricted sites in mature cardiomyocytes. At this developmental stage, HCN4 increasingly colocalizes with caveolin-3, thus providing a possible explanation for the negative shift of HCN current threshold. A similar modification of caveolin-3/HCN4 interaction occurs in native human cardiomyocytes, as suggested by a similar negative shift of HCN activation threshold characterizing the transition from fetal to adult cardiomyocytes (Bosman et al., 2013).

c. HCN expression in the developing nervous system. At variance with cardiogenesis and stem cell-derived cardiomyocytes, systematic studies investigating isoformand age-dependent changes in HCN expression levels during pre- and postnatal development of the CNS are missing, with available data referring to selected regions. In CA1 hippocampal pyramidal cell layer of embryonic rats, a robust increase of $\mathrm{HCN} 1$ transcript and protein expression occurs during development, with concomitant reduction of HCN4 and relatively stable HCN2 levels. By birth, the contribution of HCN1 to the total HCN channel pool has risen from $30 \%$ to $60 \%$. At subcellular level, the proximal-to-distal dendritic gradient of $\mathrm{HCN} 1$ is already present at postnatal day 2 (Bender et al., 2001; Surges et al., 2006; Brewster et al., 2007). Similarly, overall $I_{\mathrm{h}}$ density increases nearly sixfold in rat thalamocortical relay neurons during the first 3 months of postnatal life, accompanied by a progressive decrease in cAMP sensitivity. In keeping, quantitative analyses of HCN channel isoforms revealed a steady increase of transcript and protein expression levels of HCN1 and $\mathrm{HCN} 2$, with reduced relative abundance of $\mathrm{HCN} 4$ (Kanyshkova et al., 2009; Yoshimoto et al., 2015). An interesting observation comes from studies in the brainstem auditory neurons, where HCN channels are crucially involved in the location of sounds (Leao et al., 2006). This function is poorly present at birth in gerbils (as in humans) and undergoes intense postnatal adaption; at this stage, the onset of mature hearing with precise temporal resolution was associated with marked developmental changes in $I_{\mathrm{h}}$. In particular, the kinetics of activation-deactivation became faster and conductance larger, and activation was shifted rightward. Overall, these changes were attributable in part to an increasing role of HCN1 isoforms, and largely ( $\mathrm{V}_{1 / 2}$ shift) to the maturation of signaling pathways, in particular cAMP- and PI $(4,5)$ P2-dependent modulation (Khurana et al., 2012).

\section{B. Distribution, Adaptive, and Maladaptive Role of HCN Channels in Mammals}

1. HCN Current in the Heart: an Ideal Pharmacological Target. Excellent reviews have described the key role of cardiac $I_{\mathrm{h}}$ in automaticity and its interplay with other ion currents in the SAN (Accili et al., 2002; DiFrancesco, 2006, 2010; Biel et al., 2009), as well as in 
the conduction system (Mangoni and Nargeot, 2008). We refer the reader to these papers for a systematic discussion of physiologic aspects and to recent optical mapping (Torrente et al., 2015) and computational approaches (Fabbri et al., 2017). Overall, HCN channels contribute to two essential features of primary and subsidiary pacemakers: the appearance of a diastolic depolarization and the modulation of its steepness by the sympatho-vagal balance or other endogenous factors (Fig. 4). Indeed, a relevant fraction of the antiarrhythmic and antianginal effect of old and new drugs (from beta-blockers to digoxin and ivabradine) resides in the indirect (antiadrenergic or vagomimetic) or direct (HCN blockade) effect on $I_{\mathrm{h}}$ in pacemaker cells. According to recent computational approach modeling the human SAN AP, HCN current exerts its modulatory role mainly by changing the rate of the diastolic depolarization phase (DDR) over the first $100 \mathrm{~ms}$ following the maximum diastolic potential (Fabbri et al., 2017). This might be more relevant in species with low heart rate (e.g., humans versus rodents) because, as elegantly discussed by Zaza (2016), a nonlinear relationship exists between DDR and time: the longer the cycle length, the greater the bradycardic effect of reducing DDR (and vice versa).

a. Expression and physiologic role of HCN isoforms in cardiac regions. $I_{\mathrm{h}}$ may exhibit different electrophysiological properties (such as activation threshold and amplitude), also depending on the relative expression of different isoforms. Indeed, in the heart, HCN channels exhibit a regional specific distribution (Herrmann et al., 2012; Deng et al., 2015; Li et al., 2015a). A remarkable variation in the total amounts of HCN channels differentiates pacemaker centers from working myocardium, being $\mathrm{HCN}$ transcripts and proteins expressed at highest levels in the SAN and in the conduction system (AVN and Purkinje fibers). Additionally, isoform multiplicity differs according to species, except for $\mathrm{HCN} 3$, which displays a weak expression, regardless of regions and species. In the SAN of humans, rabbits, mice, and dogs, HCN4 is the main protein isoform compared with the others, the remaining fraction being composed by HCN2 and HCN1 in humans and HCN1 in mouse and rabbits. Differently, rat SAN expresses similar amount of HCN2 and HCN4 (Huang et al., 2016).

In mouse AVN, almost all cells express HCN1 and HCN4, whereas HCN2 is limited to some regions. The bundle of His is particularly enriched of HCN4, whereas bundle branches also display HCN1 and HCN2 (Herrmann et al., 2012). Human, rabbit, and rat AVN largely express HCN4 protein isoform, with HCN1 present in smaller amount (Dobrzynski et al., 2013).

The expression pattern of HCN protein in atria and ventricles also displays isoform variability; however, most mammalians, including humans, exhibit a prevalence of HCN4 and HCN2, followed by smaller amount of HCN1 and negligible levels of HCN3 (Lezoualc'h et al., 2007; Stillitano et al., 2008).

i. HCN in SAN Cells and Its Contribution to Cardiac Pacemaking: Membrane and Calcium Clocks. HCN channels have long been recognized for their primary role in pacemaker impulse generation and regulation. However, the specific contribution of $\mathrm{HCN}$ has considerably evolved in recent years. Two main mechanisms, the voltage clock and the $\mathrm{Ca}^{2+}$ clock, mainly sustained by $\mathrm{HCN}$ current and $\mathrm{Ca}^{2+}$ release from sarcoplasmic reticulum, respectively, are supposed to contribute to a coordinated system that jointly drives spontaneous electrical activity in SAN. The relative contribution of the two mechanisms to basal rhythm and its adaption to autonomic balance have been the object of an intense debate; to have a taste of differentsometimes conflicting-views, the reader is referred to a lively point-counterpoint exercise by the leading scientists in the field (DiFrancesco and Noble, 2012; Maltsev and Lakatta, 2012) and the enlightening accompanying comment (Rosen et al., 2012).

In brief, in the SAN during the diastolic phase, the fraction of open HCN channels provides a steady-state inward current driving membrane potential $(-70 /-40 \mathrm{mV}$, depending on cells) to depolarize toward the threshold required to generate a spontaneous AP (DiFrancesco et al., 1986). A key role of HCN channels, particularly the HCN4 isoform, is suggested-besides other argumentsby bradycardia (or severe bradycardia) consequent to the following: 1) loss of HCN4 function in KO mouse models or patients carrying HCN4 mutations (see later in this section), and 2) the effect of selective HCN blockers (see section Pharmacology of HCN Channels). It is well recognized that the steepness of the diastolic depolarization in pacemaker cells also results from the concerted (simultaneous or sequential) work of other membrane ionic conductances: NCX, L- and T-type $\mathrm{Ca}^{2+}$ channels, $\mathrm{Na}^{+} / \mathrm{K}^{+}$ATPase, voltage-dependent $\mathrm{K}^{+}$and background $\mathrm{Na}^{+}$currents - just to mention some relevant ones. The point by DiFrancesco and Noble (2012) is that these conductances concert to set the pacing rate-the membrane clock-with $I_{\mathrm{h}}$ being the conductor, also in force of its exquisite sensitivity to autonomic balance (via intracellular cAMP). Lakatta's group (Vinogradova et al., 2010) named calcium clock the periodic, spontaneous submembrane calcium release from sarcoplasmic reticulum, triggering $\mathrm{Ca}^{2+}$ extrusion via NCX current, which in turns depolarizes the membrane, activates Tand L-type calcium current, and finally triggers APs. The regular sequence of APs and its adaption to autonomic input might be the result not of a main conductor such as $I_{\mathrm{h}}$, rather of the interplay between the rate of spontaneous $\mathrm{Ca}^{2+}$ release-roughly periodic-and the fine adjustment operated by the balance of inward/outward membrane conductances, including $I_{\mathrm{h}}$. At variance with ivabradine, there are not blockers of calcium-clock players specific for SAN cells; in fact, their loss of function impairs atrial and 


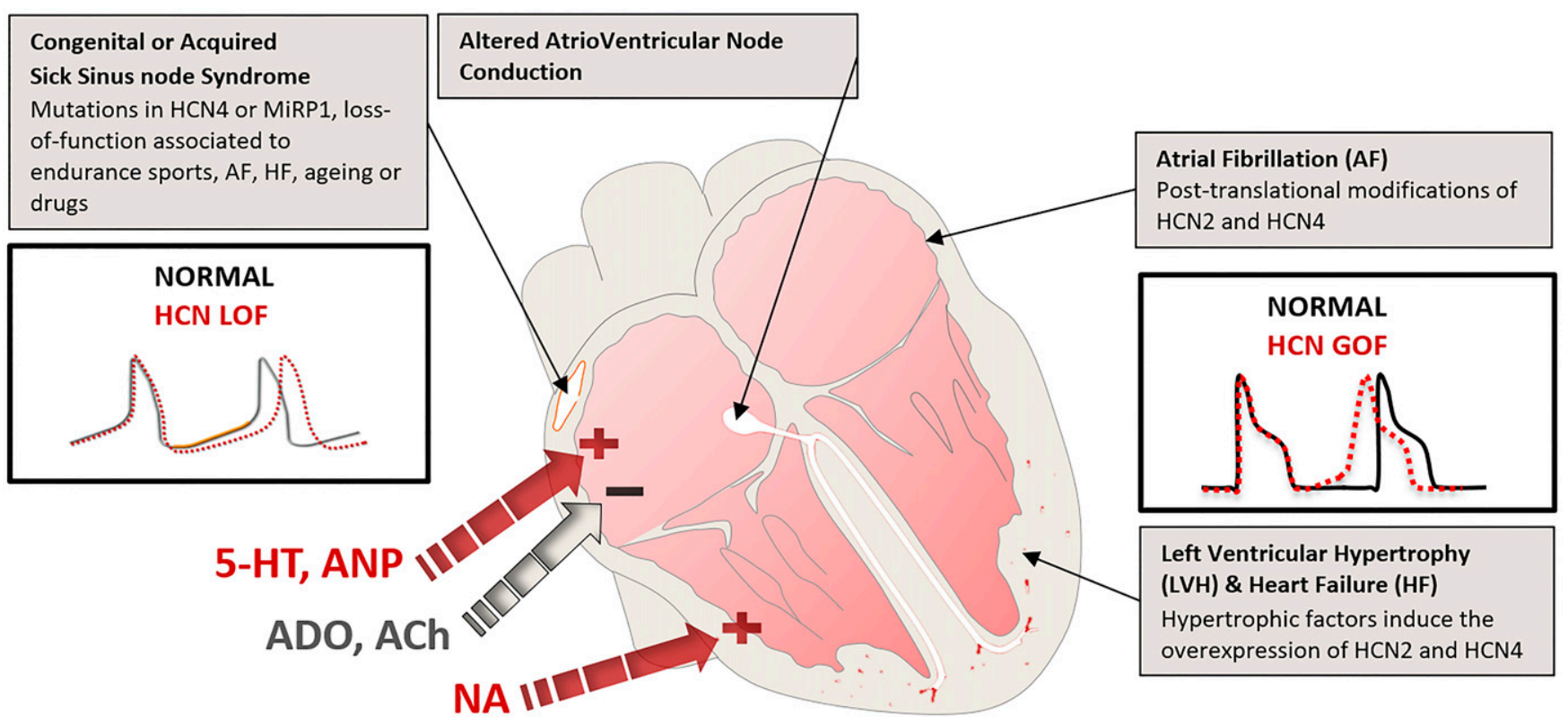

\begin{abstract}
Neurohumoral modulation of HCN channels
5-HT, ANP (atrial and nodal cells) and NA (atrial, ventricular and nodal cells) stimulate HCN activation by increasing cAMP levels while ACh and ADO cause opposite effects.

GOF or LOF caused by endogenous factors may cooperate with altered channel expression to promote arrhythmogenic alterations (brady- or tachyarrhythmias).
\end{abstract}

Fig. 4. Role of HCN channels in cardiac electrophysiological abnormalities. Pathologic implications of dysfunctional HCN channels in cardiac regions arising from transcriptional or post-transcriptional alterations of $I_{\mathrm{h}}$ due to mutations or modulation by endogenous factors. Schematic traces represent the consequences of HCN loss of function (LOF) or gain of function (GOF).

ventricular contractility in vivo. The scientific debate has been also fueled by discrepant results obtained in mouse models undergoing genetic deletion of sinoatrial HCN4, ranging from lethal bradycardia (Baruscotti et al., 2011) to minor rhythm alterations (Herrmann et al., 2007). Although experimental strategies of genetic manipulation as well as the contribution of different $\mathrm{HCN}$ isoforms may help explain different results in $\mathrm{HCN} 4-\mathrm{KO}$ mice, it is worth mentioning the following: 1) KO of STIM1, a key regulator of calcium dynamics, also causes severe bradycardia in mice (Zhang et al., 2015), and 2) complete ablation of $I_{\mathrm{f}}$ by a dominant-negative $\mathrm{HCN} 4$ mutant channel expressed in SAN cells alters membrane excitability and calcium cycling (i.e., both clocks), pacemaking and conduction impairment being partially rescued by additional $\mathrm{KO}$ of the muscarinic $\mathrm{G}$ protein-activated (GIRK4) channels (Mesirca et al., 2014). Interestingly, the latter model confirmed the prominent role of $I_{\mathrm{h}}$ in SAN as sensor of the autonomic nervous system input.

ii. HCN Expression and Function in Subsidiary Pacemakers. In the AVN the function of HCN channels does not diverge substantially; in fact, although the specific role played by $\mathrm{HCN}$ channels in this region is less investigated, robust experimental evidence indicates that HCN channels are implicated in AVN pacemaking and conduction (Liu et al., 2008; Marger et al., 2011; Verrier et al., 2014, 2015). Of note, abolition of HCN4 sensitivity through conditional expression of dominant-negative HCN4 channels lacking cAMP sensitivity reduces the spontaneous activity of AVN cells under basal conditions, but does not impair the maximal response to $\beta$-adrenergic stimulation, suggesting that HCN4 channels influence AVN basal activity, but are not obligatory for $\beta$-adrenergic regulation (Marger et al., 2011).

Transgenic mouse models have further consolidated the functions of HCN channels in SAN and AVN cells. In fact, inducible cardiac ablation of HCN4 in mice leads to progressive severe bradycardia, followed by AVN block, eventually resulting in cardiac arrest and death (Baruscotti et al., 2011).

The physiologic role of HCN channels in the healthy working myocardium remains an issue for which a conclusive function is still unclear. Since the early evidence obtained in human atrial appendage fibers, $I_{\mathrm{h}}$ has been hypothesized to support the spontaneous electrical activity observed in the atrial tissue. Subsequently, a series of studies investigated the specific properties of HCN current in atrial myocytes, showing that voltage dependence, activation kinetics, and ionic selectivity are similar to those retrieved in SAN cells (Carmeliet, 1984). The role of HCN channel in the atria differs from that in SAN because most healthy atrial cardiomyocytes have a stable resting membrane 
potential and infrequently display spontaneous electrogenesis, in line with a low contribution of $\mathrm{HCN}$ current to resting membrane potentials $(-80 /-70 \mathrm{mV})$ and absence of spontaneous automaticity. However, when the integrity of the intracellular milieu is preserved, some human atrial myocytes display a clear diastolic depolarization phase, suggesting that HCN current in the atria may overtly influence electrogenesis, in particular when favoring conditions are present, such as reduced repolarizing currents or increased adrenergic tone (Cerbai and Mugelli, 2006).

Similar observations are drawn for HCN channels in the healthy ventricle, where HCN current is readily detectable in most cells displaying a stable resting membrane potential (Cerbai and Mugelli, 2006; Sartiani et al., 2015). Interestingly, recent evidence has uncovered a different function for HCN in the mouse ventricle, where the channel appears involved in the prolongation of AP repolarization. This led to propose that HCN channels, and particularly HCN3, might mediate a depolarizing background current that regulates ventricular resting potential and counteracts the action of hyperpolarizing potassium currents in late repolarization (Fenske et al., 2011). These findings partially agree with a different study in the mouse, where HCN2 and HCN4 isoforms appear to predominate in controlling the late phase of repolarization (Hofmann et al., 2012).

\section{$b$. Altered HCN function and cardiac disease.}

i. Altered HCN Properties and Dysfunction of Sinus and Atrioventricular Nodes. The understanding of pacemaker alterations leading to cardiac arrhythmias is rapidly enlarging the genetic basis; currently, the combined efforts of clinical practice and appropriate transgenic models have helped to identify some modifications of HCN channel functions and regulatory proteins associated with dysfunctional cardiac pacemaking and/or conduction (Verkerk and Wilders, 2014).

Screening analysis performed in patients with idiopathic bradycardia has uncovered different loss-offunction mutations of HCN4 gene leading to impaired impulse generation capacity of SAN cells associated or not with conduction dysfunctions (AVN block and altered chronotropic response) (Fig. 4). Deep bradycardia and AVN block are also found in adult transgenic mice with inducible cardiac ablation of HCN4 (Baruscotti et al., 2011), further corroborating the pathophysiological implications of $\mathrm{HCN} 4$ reduction in cardiac pacemaker and conduction. Interestingly, HCN4 mutations have been identified in families with bradycardia and left ventricular noncompaction cardiomyopathy, a complex clinical phenotype that associates HCN alterations to cardiac structural abnormalities (Milano et al., 2014). Recently, a novel loss-of-function mutation of HCN4 channel has been identified during a screening in patients with sick sinus and Brugada syndromes (Biel et al., 2016), a finding that further complicates the understanding of proarrhythmic role of HCN channel dysfunctions.

In the healthy heart, a proper sensitivity of HCN4 to cAMP is also important to set basal HCN current magnitude and the contribution of $\mathrm{HCN}$ to resting pacemaker automaticity. In fact, transgenic mice expressing human mutated HCN4 gene lacking the cAMP binding site (CNBD) results in basal bradycardia and reduced heart rate sensitivity to adrenergic stimulation (Alig et al., 2009). However, the same model proved that rate adaption during physical activity is preserved, thus indicating that different mechanisms, alone or in combination with HCN channels, are enrolled to increase heart rate following adrenergic stimulation (Alig et al., 2009; Rosen et al., 2012). Of note, adrenergic regulation of heart rate is also preserved, at least partially, in condition of HCN channel blockade, because the state amplifies the effects of any autonomic stimuli on cycle length (Zaza and Lombardi, 2001).

As opposed to the above reports, a recent study in patients with inappropriate sinus tachycardia has identified a gain-of-function mutation (R524Q) in cardiac HCN4 channel. Mutant channels display a higher sensitivity to cAMP and mediate a larger than normal current during the diastolic depolarization, a finding in line with the enhanced cardiac rate detected in these patients (Baruscotti et al., 2016). As a confirmation of the role of $I_{\mathrm{h}}$ in human SAN pacemaking, a recent computational approach shows that all HCN4 mutations associated with a loss of function slow the pacemaker rate down in simulated APs, with negligible effects on other AP parameters, whereas the only gainof-function mutation described to date has opposite consequences (Fabbri et al., 2017).

Regulatory proteins also have a role in HCN channel dysfunction in pacemaker centers. Currently, a single mutation in the MiRP1/KCNE2 gene has been associated with symptomatic severe sinus bradycardia and suppression of HCN current in vitro, an effect conceivable with an altered interaction between the regulatory subunit and HCN channels (Nawathe et al., 2013).

Sinus bradycardia may also be attributed to nongenetic causes, such as those occurring physiologically with endurance training (Dobrzynski et al., 2013; D'Souza et al., 2014) or ageing (Monfredi and Boyett, 2015). Both conditions are associated with complex and still incompletely defined modifications of SAN and atria that may predispose to develop atrial tachyarrhythmias. On the other side, pathologic conditions such as AF (Jackson et al., 2017) and heart failure (Wang and Hill, 2010) may also lead to sinus node dysfunctions associated with anatomic and electrical changes.

ii. Atrial Remodelling and Fibrillation. HCN current constitutively present in the human atria has since long been proposed to sustain atrial arrhythmias 
associated with different cardiac pathologies or triggered by various modulatory signals (Opthof, 1998) (Fig. 4).

In diseased conditions, such as cardiac hypertrophy and failure, atrial dysfunction and arrhythmias may occur because of adverse remodeling triggered by chronic impairment of ventricular function. In the context of chronic heart failure, experimental data have documented an altered expression of HCN channels in right atrial tissue, where HCN4 transcript was found significantly increased (Zicha et al., 2005). In line with this study, in atrial tissue from failing human hearts, HCN2 and HCN4 transcripts and proteins were also increased and most likely related to the enhanced HCN current and the positive voltage shift of channel activation (Stillitano et al., 2008). Interestingly, in a different study (Lai et al., 1999), the overexpression of HCN2 in the left atria is positively related with left atrial filling pressure, an indicator of congestive heart failure, further corroborating the link between left ventricular dysfunction and atrial electrical remodeling.

A limited number of studies have investigated the issue in a defined arrhythmic state, such as chronic AF. In this setting, a significant increase of HCN current has been reported in atrial cardiomyocytes from diseased patients compared with control (Stillitano et al., 2013). In particular, at voltage values around myocyte resting membrane potential $(\cong-70 \mathrm{mV})$, HCN fractional activation is $10 \%$ larger (from 20\% to 30\%) because of a positive voltage shift in channel activation, suggesting that chronic AF modifies atrial electrogenesis also by enhancing the contribution of HCN current. The molecular counterpart of this modification is not obvious, consisting of unchanged transcript levels for $\mathrm{HCN} 1 / \mathrm{HCN} 2$ and reduction of $\mathrm{HCN} 4$ and MiR-1; however, protein amounts are preserved, most likely because of post-transcriptional processing. The modifications of HCN transcripts are in agreement with those reported in a previous study performed on patients in chronic AF (Lezoualc'h et al., 2007). The mechanism underlying the increase of $\mathrm{HCN}$ current in chronic AF is not clear; it is possible to hypothesize the occurrence of modifications of HCN regulatory subunits that affect channel function. In this regard, the lack of quantitative modifications of caveolin-3 possibly escludes changes of the interaction between caveolin-3 and HCN channels in the diseased atria.

At variance with the above reports, recent studies in a canine model and in humans (Li et al., 2014b, 2015b) have identified an age-related increased expression of HCN2 and HCN4 transcripts and proteins in the atria. These modifications are associated with proarrhythmic alterations in the canine atria and propensity to $\mathrm{AF}$. In humans, similar modifications were found to be associated with reduction of MiR-1 and MiR-133A that were greater in patients with $\mathrm{AF}$ (Li et al., 2015b).
The arrhythmogenic potential of adverse cardiac remodeling can be amplified or reduced by different triggers present at local or systemic levels. Some of those having important implications for $\mathrm{AF}$, such as catecholamines, serotonin, atrial natriuretic peptide (ANP), and adenosine, also modulate the function of HCN channels via activation of different types of $G$ protein-coupled receptors (Table 2). In the human atrium, serotonin and catecholamines interact with and activate $\mathrm{G}_{\mathrm{s}}$-coupled receptors, namely subtype- 4 serotonin receptor and $\beta_{1^{-}}$and $\beta_{2}$-adrenoceptors (Pino et al., 1998; Lonardo et al., 2005). Following increase of intracellular cAMP, both serotonin and catecholamines exert a stimulatory effect on HCN amplitude, shifting the activation voltage to positive values. Differently, ANP is synthesized and stored as a prohormone in the atria, and, upon distension, it is released, allowing activation of two receptors (peptide receptors $A$ and B). Both of them stimulate a guanylyl-cyclase activity, thereby increasing intracellular concentration of cGMP; in this case, intracellular cAMP level rises because of cGMP-mediated inhibition of cAMP phosphodiesterases (Lonardo et al., 2004). It has been hypothesized that, in conditions of HCN channel gain of function, as those determined by adverse remodeling, these mediators may promote the function of HCN channels at physiologic potentials, enhancing the propensity to arrhythmias. Among the observations corroborating the hypothesis are the persistence of all mediator activity and associated signaling during $\mathrm{AF}$ and the values of affinity constants of serotonin and ANP for the corresponding receptors, which are closer to the physiologic concentrations of serotonin or ANP derived by local release during platelet aggregation or cell stretching. Further investigations are needed to better clarify the pathophysiological implications of these pathways in atrial arrhythmias and to evaluate the interplay of concomitant excitatory effects exerted on HCN channels expressed in atrial myocytes and in SAN cells.

iii. Ventricular Hypertrophy and Failure. Over the last decades, much attention has focused on HCN current in nonpacemaker cells and its potential role in triggering ventricular arrhythmias. The first evidences were obtained in the spontaneous hypertensive rat, which develops age-related cardiac hypertrophy associated with several electrophysiological alterations at ventricular level, including an unusual diastolic depolarization phase (Barbieri et al., 1994). Subsequently, this peculiar trait was associated with the occurrence of an atypical $I_{\mathrm{h}}$ that resulted to linearly relate to the severity of cardiac hypertrophy (Cerbai et al., 1994). Ventricular $I_{\mathrm{h}}$ exhibits electrophysiological properties and sensitivity to pharmacological blockade similar to those described in atrial and SAN cells. However, as described for the atrial current, voltage dependence is shifted to more negative potentials compared with SAN cells, being activation threshold at approximately $-70 \mathrm{mV}$. The 


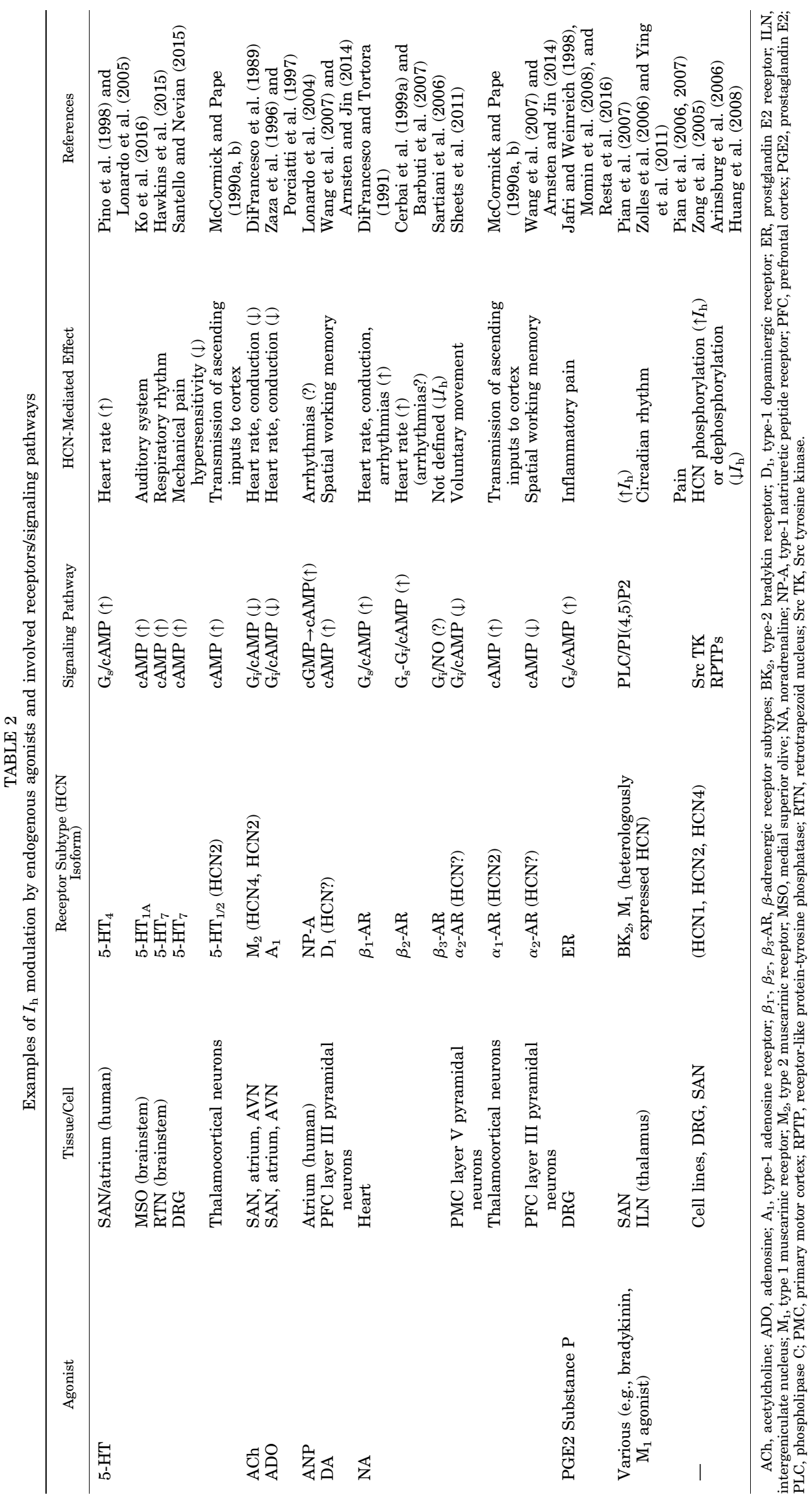


value is conceivable with a definite contribution of $\mathrm{HCN}$ current to ventricular resting membrane potential, ranging from -80 to $-90 \mathrm{mV}$. The atypical voltage dependence of HCN current in the ventricle most likely arises from specific isoform assembling into functional channels, as well as the multiplicity of secondary subunits that influence HCN current properties, analogously to what was reported for nodal and immature cardiomyocytes (Cerbai et al., 1999b; Barbuti et al., 2004, 2007, 2012; Bosman et al., 2013).

HCN current in the ventricle exhibits a typical sensitivity to autonomic transmitters, mimicking that described in the SAN cells (Cerbai et al., 1999a). Upon adrenergic stimulation, the marked positive shift of $\mathrm{HCN}$ voltage dependence most likely reflects a major contribution of HCN2 and HCN4 to tetramer assembly, being these isoforms most sensitive to cAMP-mediated effects (Stillitano et al., 2008; Wahl-Schott et al., 2014). The occurrence and density of HCN current in human cardiac ventricular myocytes isolated from explanted hearts are larger compared with control ventricle and related to disease etiology, being more prominent in ischemic than in dilated cardiomyopathy (Cerbai et al., 1997, 2001; Hoppe and Beuckelmann, 1998). In this setting, the gain of function of HCN current relates to increased levels of HCN4 and HCN2 proteins and transcripts (Fernández-Velasco et al., 2003; Stillitano et al., 2008; Suffredini et al., 2012) (Figs. 3 and 4).

Following on these pieces of evidence, a number of studies have extensively documented that increase in HCN channel expression is a common trait of different models of cardiac hypertrophy (Stilli et al., 2001; Fernández-Velasco et al., 2003) and myocardial infarction (Sartiani et al., 2006; Suffredini et al., 2012). Hence, HCN channels most likely have their place in the fetal/embryonic gene pattern re-expressed during functional remodeling of the diseased ventricle (Swynghedauw, 1999). Interestingly, the expression of the HCN channels appears more pronounced in ventricular regions with the greatest overload, suggesting the possibility of mechano-sensitive mechanism of channel expression (Fernández-Velasco et al., 2003).

Despite the emerging appraisal of HCN channel as representative marker of cardiac remodeling, its arrhythmogenic role in the ventricle has long remained unproven both in experimental and clinical studies. A recent study in a bradycardic model of heart failure has provided insight into the role of $\mathrm{HCN}$ channels in the initiation of triggered activity. The model, expressing a dominant-negative form of the transcriptional repressor neuron-restrictive silencing factor, a regulator of the fetal cardiac gene program, develops heart failure and dilated cardiomyopathy. Along this process, HCN2 and HCN4 channels are upregulated, leading to enhanced HCN current density associated with ventricular tachycardia, premature ventricular contractions, and sudden cardiac death (Kuwabara et al., 2013; Yamada et al.,
2014). Accordingly, $\beta$-adrenergic stimulation increased the susceptibility of myocytes to early after-depolarizations and spontaneous APs. These findings are in agreement with another study (Hofmann et al., 2012) showing that HCN activity increases the arrhythmogenic potential in the failing myocytes through prolongation of the repolarization phase in ventricular APs.

The consolidated knowledge on the pathways involved in cardiac remodeling has led to investigate whether abnormal neurohumoral signals, known to trigger the onset and progression of adverse cardiac modifications, are also involved in HCN channel overexpression. Among the most important signals, adrenergic and renin-angiotensin-aldosterone systems are responsible for increased levels of hormones, which are released systemically and locally (Swynghedauw, 1999; De Mello, 2004) and have a well-established role in the proarrhythmic alterations of the ventricle, such as the reduction of transient outward potassium channel and the prolongation of AP duration. Indeed, in similar experimental context, chronic administration of angiotensin II receptor antagonists, losartan and irbesartan, is effective in restoring normal electrogenesis in the ventricle, including the expression and function of HCN channels (Cerbai et al., 2000, 2003).

More recently, a different pharmacological strategy has emerged from clinical trials demonstrating the efficacy of pacing to modify positively the progression and worsening of cardiac failure (Rao et al., 2007; Fox et al., 2008). That high heart rate was an independent risk factor for cardiovascular mortality and morbidity was demonstrated since the early Framingham studies (Kannel et al., 1987). Accordingly, growing clinical evidence also prompted studies aimed at testing heart-rate-reducing drugs to reverse and/or ameliorate the functional remodeling of the hypertrophic ventricle in animal models (Dedkov et al., 2007). In line with these observations, experimental findings obtained in the postinfarcted rat demonstrate that chronic treatment of ivabradine, the only bradycardic agent in clinical use, is able to ameliorate cardiac function and to counteract the global electrophysiological remodeling of the ventricle, including the overexpression of HCN channels (Ceconi et al., 2011; Suffredini et al., 2012). The mechanism(s) responsible for the benefit of heartrate-lowering drugs in heart hypertrophy and failure will be further discussed later (section Ivabradine in Angina and Heart Failure).

2. HCN in the Central and Peripheral Nervous System.

a. Overview of HCN distribution at regional, cellular, and subcellular level. Data on HCN channel expression at regional level come from in situ hybridization and immunohistochemistry performed in brain-wide sections from both adult and developing rodent brains (Moosmang et al., 1999; Monteggia et al., 2000; Santoro et al., 2000; Bender et al., 2001; Notomi and Shigemoto, 
2004). In addition, single-cell reverse-transcription polymerase chain reaction and electron microscopy analysis have provided more detailed information about the expression pattern of HCN genes with cellular and subcellular resolution in selected CNS areas in animal models (Franz et al., 2000; Luján et al., 2005; Bender et al., 2007; Brewster et al., 2007; Huang et al., 2011; Ramakrishnan et al., 2012; Paspalas et al., 2013; Dufour et al., 2014). All four HCN isoforms are expressed in the brain. The relative abundance of each subunit depends on the area, nerve cell type, or subcellular compartment. Despite the different methodologies and animal species, all reports addressing HCN distribution show good agreement. Overall, HCN subunits are strongly expressed in the CNS and peripheral nervous system (PNS), with subunit-specific pattern. In the CNS, HCN1 is highly expressed in the neocortex, hippocampus, cerebellar cortex, brainstem, and spinal cord. HCN2 is nearly ubiquitous across the CNS, but especially abundant in thalamic and brainstem nuclei. Conversely, a limited number of areas exhibits a strong HCN4 expression, such as the olfactory bulb and the thalamus, with a distribution pattern that appears complementary to that of HCN1. The expression of HCN3 is scattered throughout the brain and modest. All isoforms, but HCN3, are present in the retina (FykKolodziej and Pourcho, 2007). In the PNS, all HCN subunits are expressed. HCN1 is the most abundant in dorsal root ganglia (DRG) (Chaplan et al., 2003), although a prominent function of HCN2 in the transmission of painful stimuli has also been reported (Emery et al., 2011) (see later). The expression of HCN channels has been found also in enteric neurons (Galligan et al., 1990) and in the spiral (Chen, 1997) and trigeminal ganglion neurons (Janigro et al., 1997).

b. Physiologic role of HCN channels in the CNS: general aspects. A large body of literature has accumulated over the past two decades on the role of $\mathrm{HCN}$ channels in nervous cells. The basic aspects of HCN channel physiology in the CNS have been the topic of recent reviews (He et al., 2014; Shah, 2016). We will provide a brief overview of HCN channel function in neuronal physiology and then focus on selected contexts of specific relevance for the purpose of this review. HCN current is a major determinant of neuronal excitability in many neuronal types. The action of HCN channels on neuronal excitability arises from four fundamental properties: 1) selectivity for sodium and potassium; 2) activation curve at potentials close to firing threshold; 3) reversal potential at depolarized values $(\sim-35 \mathrm{mV})$ with respect to resting membrane potential; and 4) slow gating kinetics. Because of these properties, HCN channels generate a tonic depolarizing current, which drives the membrane potential toward firing threshold. Furthermore, HCN current offsets both negative and positive perturbations in membrane potential by responding with increased activation or deactivation, respectively.
Due to the slow kinetics, this filtering action is effective for low-frequency oscillatory inputs (high-pass filtering). Finally, modulation by the action of cAMP, PKA, PI(4,5)P2, or ancillary subunits adds to the complexity and diversity of functions of HCN channels in the CNS.

i. Regulation of Resting Membrane Potential and Intrinsic Excitability. The influence exerted by HCN current on the intrinsic excitability of the neuron has a complex nature. At $-65 \mathrm{mV}$, a fraction of $\mathrm{HCN}$ channels is tonically open (Kase and Imoto, 2012). This results in persistent sodium inflow, which depolarizes the membrane and counteracts hyperpolarization. Depolarization causes channel deactivation, and the consequent pause in current flow has a hyperpolarizing effect. Hence, by opposing negative and positive perturbations, tonic HCN current stabilizes membrane potential in the subthreshold range. Therefore, HCN loss of function causes membrane hyperpolarization and reduction of intrinsic excitability. At the same time, however, input resistance at subthreshold potentials increases and the neuron becomes more responsive toward synaptic stimulation. This dual action exerted by HCN current on neuronal function has been clearly established in many diverse brain areas, including the thalamus, the hippocampus, and the cerebellum, and has important repercussions on higher-order brain functions such as learning and memory, control of circadian rhythm, and sleep/wakefulness state (McCormick and Pape, 1990b; Maccaferri et al., 1993; Gasparini and DiFrancesco, 1997; Nolan et al., 2003). Very recently, a novel role for HCN1 in the control of the neuron's intrinsic excitability has been proposed. In the axon initial segment of medial superior olive principal neurons, HCN1-mediated current reduces spike probability by elevating the firing threshold. The $5-\mathrm{HT}_{1 \mathrm{~A}}$ receptor signaling shifts HCN channel activation curve in the hyperpolarizing direction, thereby relieving the brake on spike probability (Ko et al., 2016). Serotonindependent modulation of HCN current is also involved in the control of respiratory rhythm in the retrotrapezoid nucleus. Activation of the $5-\mathrm{HT}_{7}$ receptor causes a cAMPdependent depolarizing shift in the activation curve of HCN current, increasing the firing rate of retrotrapezoid nucleus neurons in vitro and respiratory rhythm in vivo (Hawkins et al., 2015).

ii. Rhythmogenesis. In a functional interplay with other conductances, the activation/deactivation cycle of HCN channels sets the pace of subthreshold membrane oscillations, which may eventually generate AP firing. HCN current cooperates with a subthreshold, persistent $\mathrm{Na}^{+}$current to determine a $4-10 \mathrm{~Hz}$ ( $\theta$-like) rhythm in layer II grid cells of the entorhinal cortex (EC) (Alonso and Llinas, 1989). Entorhinal grid cells show periodic, hexagonally-patterned firing locations that scale up progressively along the dorsal-ventral axis of medial EC, which are critically involved in spatial navigation (Moser et al., 2015). In this structure, relative HCN1/HCN2 
expression ratio goes down, along with oscillation frequency, following a dorsal-ventral gradient (Notomi and Shigemoto, 2004). This frequency gradient is consistent with the distinct time constants of HCN1 and HCN2 isoforms. In HCN1-KO mice, the dorsal-ventral gradient of the grid pattern is preserved, but the size and spacing of the grid fields, as well as the period of the accompanying $\theta$ modulation, are expanded (Giocomo and Hasselmo, 2009; Giocomo et al., 2011). In thalamocortical neurons, HCN current mainly results from HCN2 expression. Adrenergic and serotonergic stimulation causes a cAMP-dependent modulation of HCN2mediated current, which in turn determines the firing mode of thalamocortical neurons. During wakefulness and rapid eye movement sleep, thalamocortical neurons fire in a single-spike, or transmission mode. In this state, information is effectively transmitted to the cortex. During non-rapid eye movement sleep and absence seizures, thalamocortical neurons fire in a burst mode, and transfer of signals to the cortex is believed to be not as effective (McCormick and Pape, 1990a, b). In agreement, global HCN2 deletion causes absence epilepsy with typical spike-and-wave discharges in electroencephalogram (EEG) recordings (Ludwig et al., 2003). In the intergeniculate leaflet neurons, a retino-recipient thalamic structure implicated in orchestrating circadian rhythm, a role for HCN3 subunit has been described. In this work, HCN3-mediated current drives low-threshold burst firing and spontaneous oscillations and is bidirectionally modulated by $\mathrm{PI}(4,5) \mathrm{P} 2$. Depletion of $\mathrm{PI}(4,5) \mathrm{P} 2$ or pharmacologic block of HCN current results in a profound inhibition of excitability (Ying et al., 2011).

iii. Synaptic Excitability and Plasticity. Functional HCN channels are strongly expressed along the dendritic arborizations of neocortical and hippocampal neurons with a soma-to-dendrites expression gradient (Bender et al., 2001; Lörincz et al., 2002; Harnett et al., 2015). In these areas, HCN current constitutes a shunt conductance accelerating the membrane's time constant and the decay phase of excitatory postsynaptic potentials (EPSPs). As a corollary, temporal summation during an EPSP sequence is minimized. This function, discovered and characterized in pyramidal neurons of the hippocampal CA1 region and somatosensory cortex (Magee, 1998, 1999; Williams and Stuart, 2000; Berger et al., 2003), has then been described in subcortical structures (Ying et al., 2007; Engel and Seutin, 2015; Masi et al., 2015). In the dendritic compartment of CA1 pyramidal neurons, the shunting effect exerted by HCN current limits the activation of voltage-dependent calcium entry, with important consequences on synaptic excitability (Tsay et al., 2007). The nonuniform distribution of HCN current is also responsible for a phenomenon termed "site independence" of synaptic potentials, whereby the decay time constant of distally-generated EPSPs is similar to that of proximally-generated EPSPs (Williams and Stuart, 2000). As a rule, HCN current accelerates the kinetics of both forward- and backward-propagating depolarization waves, thus increasing the precision with which the dendrite detects the concurrence of salient events, including EPSP-AP coincidence, an event leading to plasticity (Pavlov et al., 2011). In several brain areas, long-term potentiation and associated cognitive functions may be constrained by normal HCN function. Indeed, HCN1-KO mice show improved hippocampal-dependent learning and memory performance. In these mice, proximal synapses (CA3-CA1 contacts) function normally, whereas they are potentiated at distal locations (EC layer III-CA1 contacts), as predicted by stronger expression of HCN channels in this compartment (Nolan et al., 2004). HCN current affects cortical functions such as cognition, movement planning, and execution by setting the connectivity strength within local cortical microcircuits. HCN current is abundantly expressed in dorsal-lateral prefrontal cortex (PFC) layer III neurons, a population crucially involved in spatial working memory. In these neurons, HCN channels are modulated by the opposite action of $\alpha_{2}$-adrenergic receptor $\left(\alpha_{2}\right.$-AR) and dopaminergic receptor type 1 (D1) stimulation, via cAMP signaling. When $\alpha_{2}$-AR stimulation inhibits cAMP-HCN channel signaling, neurons are more tightly connected to recurring microcircuit activity and performance is optimal. In contrast, exposure to stress causes a D1-mediated enhancement of cAMP-HCN activity, leading to a functional disconnection from the local network and impairment of spatial working memory (Wang et al., 2007; Arnsten and Jin, 2014). In the mouse primary motor cortex, HCN expression is specifically elevated in corticospinal neurons of layer V. HCN current confers these neurons a $4 \mathrm{~Hz}-$ resonance preference and gates synaptic inputs from layer II/III pyramidal neurons, determining the efficacy of signal transmission between the two layers. In this context as well, $\alpha_{2}$-AR stimulation modulates $I_{\mathrm{h}}$ function (Sheets et al., 2011).

iv. Resonance Properties. HCN current in the dendritic compartment also confers the neuron-specific resonance properties, i.e., the ability to respond preferentially to inputs at a certain frequency, and thus determines to what extent the neuron responds to synchronous network activity. Due to its slow gating kinetics, HCN current has high-pass filtering properties that, combined with the low-pass filtering action exerted by membrane time constant (Bedard et al., 2006 ), result in a resonance preference of $1-10 \mathrm{~Hz}$ (Hutcheon et al., 1996). The increased power of $\theta$ oscillation due to HCN1 deletion and potentiation of perforant path-CA1 pyramidal neurons synapse has been proposed as the physiologic background of hippocampal-dependent learning and memory storage (Nolan et al., 2004). The ability to resonate in a HCNdependent manner has been reported for other areas, but the functional significance at higher-order level has 
yet to be established (Ulrich, 2002; Wang et al., 2006; Xue et al., 2012b; Borel et al., 2013).

$v$. Neurotransmitter Release. HCN current at synaptic terminals has been reported to control efficacy of vesicle release. $\mathrm{HCN} 1$ and $\mathrm{HCN} 2$ isoforms are present at GABAergic terminals of pallidal axon collaterals (Boyes et al., 2007) as well as glutamatergic terminals onto EC layer III pyramidal neurons (Huang et al., 2011). In both settings, pharmacological or genetic inactivation leads to elevation of spontaneous synaptic release. Huang et al. (2011) have suggested that HCN current inhibitory action is exerted by setting the potential to values where Cav3.2 N-type channels are less active. A follow-up study from the same authors shows that HCN1 channels restrict the rate of exocytosis from a subset of cortical synaptic terminals within the EC and constrain non-APdependent and AP-dependent spontaneous release, as well as synchronous, evoked release (Huang et al., 2017).

Despite a large mass of information on the cellular effects of HCN channels, our knowledge of their role in more integrate CNS functions and behavior is rather indirect and derived from pathophysiological studies, as described below. An interesting insight comes from recent evidence in Aplysia californica, whose neuroanatomy and physiology are by far less complex than the mammalian brain (Yang et al., 2015). A. californica motor neurons possess only one $\mathrm{HCN}$ isoform, acHCN; $I_{\mathrm{h}}$ appears to be involved in classic conditioning upon stimulation by nitric oxide/cGMP signaling and subsequent enhancement of a $N$-methyl-D-aspartate (NMDA)-like current pathway, similarly to the mammalian hippocampal neurons (Neitz et al., 2014).

c. HCN channels in pathologic states of the CNS. As discussed earlier, HCN current affects the excitability of many neuronal types in a complex manner. Figure 5 exemplifies the major pathophysiological implications of dysfunctional HCN channels in CNS and PNS described to date.

$i$. Epilepsy. The majority of studies have reported that increased neuronal excitability, eventually leading to epileptic state, is associated with loss-of-function mutations in HCN1 and HCN2 isoforms (Nava et al., 2014; DiFrancesco and DiFrancesco, 2015). HCN1-null mice are more susceptible to kainic acid-induced seizures, and, in spite of the hyperpolarization in resting membrane potential, synaptic excitability of cortical and hippocampal neurons is increased in these mice (Huang et al., 2009). Global HCN2 deletion results in the appearance of absence seizures (Ludwig et al., 2003). Mice carrying a $\mathrm{C}$ terminus-truncated HCN2 protein manifest generalized spike-wave absence seizures. Next-generation sequencing analysis on patients' cohorts has revealed association with single-point mutations leading to loss-of-function phenotype in HCN2 isoform and idiopathic generalized epilepsy (Tang et al., 2008; DiFrancesco et al., 2011). Another study, however, reported a single-point mutation in HCN2 gene leading to a gain of function, showing a significant association with febrile seizures (Dibbens et al., 2010). In agreement with the hypothesis that epilepsy correlates with a negative functional modulation of $\mathrm{HCN}$ current is the evidence that the established anticonvulsants such as lamotrigine (Poolos et al., 2002), gabapentin (Surges et al., 2003), and acetazolamide (Munsch and Pape, 1999) activate HCN current in vitro. However, it is not clear whether HCN current upregulation contributes to the antiepileptic efficacy of these compounds. HCN current loss of function may also result from alterations in the expression of auxiliary subunits, such as TRIP8b. TRIP8b-KO mice feature spontaneous spike-wave discharges on EEG that are the electrographic hallmark of absence seizures, resembling the scenario of a global HCN2 deletion. Compared with global HCN2 KOs, TRIP8b KOs have no cardiac phenotype and less severe seizure phenotype. Mechanistically, TRIP8b-KO mice have significantly reduced HCN channel expression and function in thalamic-projecting cortical layer $5 \mathrm{~b}$ neurons and thalamic relay neurons, but preserved function in inhibitory neurons of the reticular thalamic nucleus (Heuermann et al., 2016). Finally, a number of studies report a remodelling of HCN1/2 expression following experimental seizure induction, suggesting a reciprocal cause-effect relation between HCN channels and epilepsy (Brewster et al., 2002; Bender et al., 2003). HCN channels are upregulated in rat CA1 pyramidal neurons following hyperthermiainduced seizures. Interestingly, an adaptive increase in GABAergic activity has been reported in this paradigm. GABAergic inhibitory postsynaptic potentials at high frequency $(\sim 50 \mathrm{~Hz})$ tend to summate and to activate HCN channels enough to cause rebound firing at the end of the inhibitory train. This mechanism has been proposed as an explanation for the paradoxical increase in GABAergic inhibition in experimental models of epilepsy (Chen et al., 2001; DyhrfjeldJohnsen et al., 2009). In summary, a relatively large number of preclinical and human genetic studies supports the evidence linking HCN channelopathy to epilepsy. These studies do not always hint at a univocal cause-effect relation between HCN alteration and disease. In fact, both up- and downregulation have been reported in association to epileptic states, and HCN expression remodelling is sometimes a consequence rather than the cause of seizures. For these reasons, the hypothesis that HCN channels may serve as new-generation targets for the development of antiepileptic drugs, yet promising, deserves further experimental testing.

ii. Autism Spectrum Disorder. It has been recently established that a reduction in $I_{\mathrm{h}}$ density is one of the alterations determined by Shank3 haploinsufficiency in reprogrammed human neurons (Yi et al., 2016). Shank3 is a ubiquitous scaffolding protein enriched at postsynaptic densities associated with autism spectrum 


Neuropsychiatric disorders
Aberrant HCN function has been
reported in animal models of
major neuropsychiatric
conditions such as
schizophrenia,
ASD, depression, anxiety and
drug abuse.

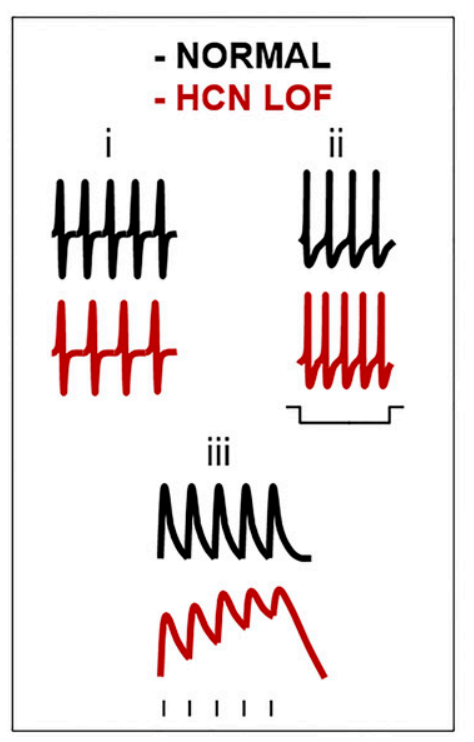

Alzheimer's disease Diminished HCN1 expression in the temporal lobe of aging macaques and $A D$ patients confers proneness to epileptic seizures.

Parkinson's disease MPP+ suppresses HCN current increasing synaptic excitability of SNc DA neurons. HCN current density is reduced in SNc DA neurons of MitoPark mice before the onset of symptoms.

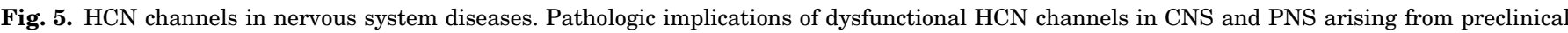

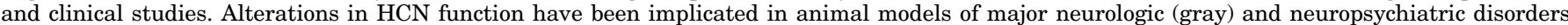

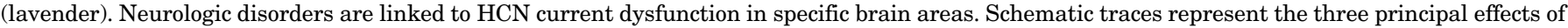
HCN loss of function (LOF) on intrinsic firing (i), stimulated firing (ii), and synaptic input integration (iii).

disorder and other neuropsychiatric disorders. Similar reduction in $I_{\mathrm{h}}$ density was found in hippocampal neurons from transgenic mice carrying a similar Shank3 mutation. Finally, chronic treatment of developing mouse neurons with ZD7288 reproduces some of the morphologic abnormalities caused by mutant Shank3.

iii. Schizophrenia. Schizophrenia is characterized by deficits in PFC function and alteration in cAMP signaling pathway. In layer II/III of the PFC, HCN1 and cAMP/phosphodiesterase signaling machinery colocalizes to the neck of thin spines to regulate gating of afferent inputs during the execution of working memory tasks. As discussed earlier, unbalance of cAMP-HCN signaling results in network disconnection and reduced performance in these tasks (Arnsten, 2011). Consistently, D1 stimulation in rat or monkey PFC impairs working memory performance, and HCN channel blockade in PFC prevents this impairment in rats exposed to either stress or D1 receptor stimulation. These findings suggest that D1 stimulation or stress weakens PFC function via opening of $\mathrm{HCN}$ channels at network synapses. This effect can be counterbalanced by elevation of cAMP levels with type- 4 phosphodiesterase inhibitors (Paspalas et al., 2013; Gamo et al., 2015). Rescue of synaptic connectivity in the PFC seems to be the rationale at the basis of the clinical efficacy of the $\alpha_{2 \mathrm{~A}}$ agonist guanfacine in PFC-related disorders (Arnsten and Jin, 2014).

iv. Substances of Abuse and Addiction. As a conductance involved in the rhythmic activity of the DA reward system, HCN current has been implicated in pathophysiology of addiction. Modulation of HCN current has been associated with exposure to ethanol (Okamoto et al., 2006), cocaine (Arencibia-Albite et al., 2012, 2017; Goertz et al., 2015), and methamphetamine (Gonzalez et al., 2016). It has recently been proposed that the reported impairment of long-term potentiation and spatial memory formation caused by cannabinoids requires activation of cannabinoid receptor type 1 and cGMP-dependent upregulation of HCN current in superficial CA1 pyramidal cells (Maroso et al., 2016).

$v$. Mood Disorders. Recent studies have suggested a role for HCN channels in animal models of depression. Chetkovich and coworkers (Lewis et al., 2011) have 
generated a KO mouse lacking the HCN channel auxiliary subunit TRIP8b that significantly regulates the voltage gating and kinetics of $I_{\mathrm{h}}$. TRIP8b deletion dramatically reduces $I_{\mathrm{h}}$ expression, disrupting subcellular distribution, in hippocampal pyramidal neurons. Behaviorally, mice lacking TRIP8b demonstrate motorlearning deficits and enhanced resistance to multiple tasks of despair with high predictive validity for antidepressant efficacy. Of note, similar resistance to behavioral despair was observed in distinct mutant mice lacking HCN1 or HCN2 (Lewis et al., 2011). Similarly, another study has shown that HCN current-mediated depressant effects are localized in the dorsal hippocampus, as focal infusion of lentiviral particles carrying HCN1 short hairpin RNA has anxiolytic- and antidepressant-like effects. Indeed, knocking down HCN1 channels increases cellular excitability and results in physiologic changes consistent with a reduction of HCN current. Moreover, treated animals display antidepressant- and anxiolytic-like behaviors associated with widespread enhancement of hippocampal activity and upregulation of brain-derived neurotrophic factor-mammalian target ofrapamycin signaling pathways (Kim et al., 2012). HCN-dependent hyperactivity of ventral tegmental area (VTA) DA neurons has been causally associated with susceptibility in social defeat stress paradigms in rats and mice. In the rat study, chronic treatment with the selective serotonin reuptake inhibitor and antidepressant fluoxetine, besides exerting the expected effects, normalizes both HCN current amplitude and VTA firing rate, suggesting a link between the actions at behavioral and cellular levels. The effects of fluoxetine are in part mimicked by intracerebral infusion of ZD7288, thus suggesting a mechanistic involvement of HCN current in the development of depressive state associated with social defeat stress (Cao et al., 2010). In the mouse, upregulated HCN current causes hyperactivity of VTA DA neurons and susceptibility to depression. Paradoxically, further enhancement of HCN function with lamotrigine or optogenetic hyperactivation of VTA discharge rate is able to rescue susceptibility to social defeat stress (Friedman et al., 2014).

vi. Fear and Anxiety. HCN currents control the excitability of principal neurons in the basolateral amygdala by reducing synaptic excitability (Park et al., 2007). In a recent study, HCN1 was identified in one of three loci associated with conditioned fear acquisition and expression in a mouse genetic reference panel. This discovery was validated using behavioral pharmacology and revealed that $\mathrm{HCN}$ current in the basolateral amygdala is required for fear acquisition and expression (Knoll et al., 2016). Collectively, these data indicate that HCN channels support negative mood states in animal models in area- and subunit-specific fashion. Direct or indirect negative modulation of $\mathrm{HCN}$ current in the brain areas subtending anxiety level, fear, and depression may have a potential therapeutic efficacy. vii. Neurodegenerative Disorders. Parkinson disease (PD) is caused by the progressive degeneration of nigrostriatal DA neurons and resulting fall of dopamine levels. In midbrain DA neurons, HCN current results from HCN2 and HCN4 expression, and substantia nigra pars compacta (SNc) DA neurons show the highest expression levels (Neuhoff et al., 2002; Dufour et al., 2014). Interestingly, HCN current density is reduced in SNc DA neurons of MitoPark mice, a genetic mouse model in which a DA-targeted mitochondrial defect leads to selective nigrostriatal degeneration and PD-like phenotype (Good et al., 2011; Branch et al., 2016). Of note, this defect marks early, asymptomatic disease stages and is not due to HCN transcript downregulation. In addition, it has been reported that 1-methyl-4-phenylpyridinium, a toxin capable of inducing PD-like selective nigrostriatal degeneration in primates and rodents, is a voltage-dependent inhibitor of HCN channels in SNc DA neurons. The 1-methyl-4phenylpyridinium-induced HCN block results in increased dendritic excitability and overall responsiveness to excitatory synaptic inputs (Masi et al., 2013). In agreement with relative HCN expression levels, the effect of HCN block on synaptic excitability is significantly greater in SNc compared with VTA DA neurons (Masi et al., 2015). Although preliminary, these findings suggest that altered HCN channel expression or function in experimental PD models deserves consideration in the context of differential vulnerability. A single study has investigated the electrophysiological actions of riluzole, a medication used to retard the onset of respiratory impairment in the treatment of amyotrophic lateral sclerosis, on the firing activity of hypoglossal motor neurons in rat brain slices, and reported that HCN current undergoes a negative shift in the activation curve following drug administration. HCN inactivation contributes to the riluzole-induced reduction in firing rate of hypoglossal motor neurons, the cellular mechanism regarded as the presumed basis of its therapeutic action (Bellingham, 2013). One study has described that HCN1 levels diminished in the temporal lobe of macaques during aging and Alzheimer's disease patients. The study also suggests a causal role of HCN1 downregulation in the increased susceptibility of Alzheimer's disease patients to epileptic seizures (Saito et al., 2012).

d. Retinal cells. In situ hybridization studies have shown high levels of HCN1 in retinal photoreceptors, whereas HCN2, 3, and 4 were not initially found in these cells (Moosmang et al., 2001). However, later studies have demonstrated that all four HCN channel isoforms (HCN1-4) were differentially expressed in different classes of retinal bipolar cells particularly clustered at synaptic terminals between bipolar cells and photoreceptors, thus suggesting a HCN possible role in the control of transmitter release (Muller et al., 2003). In particular, it has been suggested that $I_{\mathrm{h}}$ is tonically active in rod bipolar cells in darkness, where it 
quickens rod bipolar cell responses to dim light stimuli, thus partly explaining the visual side effects of HCN inhibitors (Cangiano et al., 2007).

$i$. Retinitis Pigmentosa. Although HCN are expressed in the retina, HCN dysfunction was not associated with any retinal disorder until recently. It has been known that patients during prolonged treatment with $I_{\mathrm{h}}$ inhibitor ivabradine experienced visual symptoms such as phosphenes (described as sensations of enhanced brightness in a fully maintained visual field) (Cervetto et al., 2007). However, these symptoms were dose dependent and reverted to normal 1 week after discontinuation of ivabradine without any alteration of retinal morphology and modification of HCN distribution in healthy animals (Della Santina et al., 2010). Moreover, HCN inhibition in dystrophic mice had no effect on either extent or progression of retinal degeneration. However, more recent observations pointed out the importance of HCN1 in a mouse model of CNGB1-linked retinitis pigmentosa in the progression of the disease. Indeed, the absence of HCN1 in CNGB1-KO mice exacerbated photoreceptor degeneration, thus identifying HCN1 as a major modifier of photoreceptor degeneration. This observation suggested that pharmacological inhibition of HCN channels may enhance disease progression in retinitis pigmentosa and achromatopsia (Schön et al., 2016).

\section{e. Dorsal root ganglia.}

i. Synaptic Transmission in Dorsal Root Ganglion Neurons. Shortly after the first report of a hyperpolarization-activated queer current in hippocampal neurons (Halliwell and Adams, 1982), $I_{\mathrm{h}}$ was described also in neuronal cells of the embryonic mouse DRG (Mayer and Westbrook, 1983). In these neurons, it was noticed the presence of a time- and voltagedependent conductance activated by membrane hyperpolarization owning similar characteristics of the "pacemaker current," $I_{\mathrm{f}}$, identified in SAN cells a few years before (Brown et al., 1979; Yanagihara and Irisawa, 1980). Its expression was shown to be most prominent in largeand medium-sized neurons, whereas only half of small sized DRG neurons displayed a functional $I_{\mathrm{h}}$ (Scroggs et al., 1994). Of all the four HCN isoforms, HCN1 and HCN2 are predominantly expressed in DRGs, whereas expression of HCN3 and HCN4 is low or undetectable (Acosta et al., 2012). In particular, HCN2 is mainly abundant in small-sized neurons, whereas HCN1 is the predominant subunit expressed in large neurons (Schnorr et al., 2014). Consistently, $I_{\mathrm{h}}$ in large- and medium-sized DRG neurons has a faster activation kinetic and is less cAMP-sensitive as compared with the characteristics of the current in small-diameter neurons (Gao et al., 2012).

HCN1- and HCN2-KO studies largely confirm HCN distribution in different DRG neuronal subtypes. Specifically, HCN1 deletion erased $I_{\mathrm{h}}$ in large DRG neurons, whereas slowly activating cAMP-sensitive $I_{\mathrm{h}}$ was still present in smaller DRG neurons (Momin et al., 2008). In contrast, genetic deletion of HCN2 removed the cAMP-sensitive component of $I_{\mathrm{h}}$ and abolished AP firing caused by an elevation of cAMP in nociceptors (Emery et al., 2011).

ii. HCN as Pacemakers of Pain. The precise role of $I_{\mathrm{h}}$ in DRG both in physiology and in pathology is not perfectly understood. Indeed, healthy sensory neurons seem largely unaffected by HCN blockade. Moreover, clinical studies with $I_{\mathrm{h}}$ blocker ivabradine did not show any adverse drug reaction such as dysesthesias and paresthesias (Herrmann et al., 2015). Therefore, $I_{\mathrm{h}}$ function seems to be mainly affected following pathologic states, such as in neuronal damage or in experimental model of inflammation (Papp et al., 2010; Acosta et al., 2012; Weng et al., 2012; Schnorr et al., 2014). Indeed, in these scenarios, HCN channels have been reported to increase their expression and/or function (Chaplan et al., 2003; Yao et al., 2003; Jiang et al., 2008). These observations suggested an association between HCN overexpression/overfunction and pathologic conditions, such as allodynia and hyperalgesia (Herrmann et al., 2015). Multiple mechanisms most likely underlie the phenomenon, including the following: 1) increased and modified gene expression (Papp et al., 2010; Descoeur et al., 2011; Schnorr et al., 2014); 2) $\mathrm{PI}(4,5) \mathrm{P} 2$-mediated pathway interaction (Pian et al., 2006, 2007); 3) PKA overactivation (Cheng and Zhou, 2013); and 4) elevated intracellular levels of cAMP triggered by inflammatory substances such as prostaglandin E2, serotonin, and substance P (Jafri and Weinreich, 1998; Momin et al., 2008; Resta et al., 2016).

3. HCN in Other Peripheral Districts. In rat kidney medulla, HCN1, HCN2, and HCN4 isoforms are present at transcript and protein levels. They partially colocalized with the $\mathrm{Na}^{+} / \mathrm{K}^{+}$-ATPase at the basolateral membrane, suggesting a potential link to $\mathrm{Na}^{+}$and $\mathrm{K}^{+}$homeostasis. However, channels in this tissue mediate a HCN-like cationic current with different properties compared with HCN current described in neuronal and cardiac cells, including lack of blockade by $\mathrm{Cs}^{+}$and ZD7288 (Bolívar et al., 2008), atypical properties that require further investigation. A recent study has identified the expression of HCN1 and HCN3 channels in rat nephron proximal tubule and that of HCN3 in the thick ascending limb of Henle. In this region, the channel most likely plays a role in the modulation of $\mathrm{Na}^{+} / \mathrm{K}^{+}$-ATPase activity and in acid-base homeostasis (Lopez-Gonzalez et al., 2016).

In the upper urinary tract of mice, optical mapping and video microscopy analysis have shown that HCN channel is involved in the pacemaker depolarization that initiates spontaneous peristalsis. Pacemaker potentials appear to originate in cells expressing HCN3 channels located in the pelvis-kidney junction; here, they couple to smooth muscle via gap junctions and trigger ureter peristalsis. Furthermore, HCN3-positive cells coexpress T-type calcium channels that jointly 
regulate the origin and rate of peristaltic activity (Hurtado et al., 2014; Hashitani et al., 2017).

In rat urinary bladder, all $\mathrm{HCN}$ isoforms are present. HCN1, the most prominent isoform, displays an immunolocalization restricted to interstitial cells of Cajal. Based on channel blockade by ZD7288, the function of HCN channels in these cells seems related to muscle relaxation (He et al., 2012). Following studies have confirmed the presence of all HCN isoforms in interstitial cells of Cajal of human bladder (Xue et al., 2012a). Recently, in a model of bladder hyperactivity, a higher expression of all HCN channel isoforms has been reported in association with increased current conductance; this finding suggests that $I_{\mathrm{h}}$ might play an important role in bladder dysfunctions (Deng et al., 2015). Based on the inhibitory effect of ZD7288, HCN channels might be also involved in uterine contractions in term-pregnant rats (Alotaibi et al., 2017).

Similarly to the urinary tract, a functional role has been postulated for HCN channels in smooth muscle cells from various vascular districts, such as the portal vein (Greenwood and Prestwich, 2002) and lymphatic vasa (McCloskey et al., 1999). HCN4 expression has been detected in endothelial populations in the aorta, pulmonary artery, and coronary arteries at specific developmental stages (Liang et al., 2013); however, the role of $I_{\mathrm{h}}$ in endothelial function remains elusive. More defined is the (dys)function of HCN in baroreceptor neurons; in the aortic ganglion, HCN downregulation appears to contribute to blunted excitability in diabetic rats ( $\mathrm{Li}$ et al., 2008b; Li and Zheng, 2011).

The functional expression of HCN channels in pancreatic $\beta$-cells (El-Kholy et al., 2007; Zhang et al., 2009) and $\alpha$-cells (Zhang et al., 2008) has been demonstrated several years ago by using electrophysiological and pharmacological approaches. In both cell types, channels have been postulated to modulate hormone secretion, although the relevance of HCN blockade in preventing insulin secretion appears only at low extracellular potassium concentration. This observation might be relevant to tone down the risk of adverse effect of $I_{\mathrm{h}}$ blockers. A hyperpolarization-activated current has been described in interstitial cells of Cajal, which act as pacemaker cells in the gastrointestinal tract and transduce mechanical and neurohumoral signals to smooth muscle cells. However, the role of HCN channels is strictly dependent on their regional distribution: $I_{\mathrm{h}}$ blockers inhibit and cAMPmediated signaling enhance pacemaking in colonic, but not intestinal, Cajal cells (Shahi et al., 2014). The pharmacological relevance of $I_{\mathrm{h}}$ modulation in gut motility abnormalities has not been investigated.

\section{Pharmacology of HCN Channels}

\section{A. HCN Modulation by Cyclic Nucleotides}

1. Lessons from Crystallography. Up to December 2016, 22 different structures of the intracellular
C-terminal domain have been deposited in the Protein Data Bank; of these, 20 were obtained from X-ray diffraction and two from solution nuclear magnetic resonance (NMR) (Table 3). The first crystal structures of the HCN2 C-terminal domain, in complex with cAMP and cGMP, were solved in 2003, allowing a detailed description of this portion (Zagotta et al., 2003). The cytosolic region contains two domains, the C-linker and the CNBD; the former connects the latter to the S6 transmembrane fragment. The C-linker consists of six $\alpha$-helices $\left(\mathrm{A}^{\prime}-\mathrm{F}^{\prime}\right)$ of variable length. The $\mathrm{CNBD}$ is formed by four helices (A, P, B, C), with eight $\beta$-strands arranged in a jelly roll-like topology between helix A and $\mathrm{B}$; a phosphate-binding cassette is located between $\beta$-strands 6 and 7 , and include the short $\mathrm{P}$ helix. The C-terminal domains assemble as tetramers, with a large subunit-subunit interface almost completely formed by the $\mathrm{C}$-linker region: the first two helices $\left(\mathrm{A}^{\prime}, \mathrm{B}^{\prime}\right)$ form a helix-turn-helix motif that interacts with the neighboring subunits $\left(\mathrm{C}^{\prime}\right.$ and $\mathrm{D}^{\prime}$ helices). Cyclic nucleotides establish a series of interactions within the CNBD, involving residues either in $\beta$-strands or in helices. The negatively charged cyclic phosphate is engaged in an ionic interaction with R591, whose mutation largely decreased cAMP affinity (Chen et al., 2001b); the two oxygens of the phosphate form H-bond with the $\mathrm{NH}$ of C584 $\left(\mathrm{O}_{\text {eq }}\right)$ and of T592 $\left(\mathrm{O}_{\mathrm{ax}}\right)$. The $2^{\prime}-\mathrm{OH}$ is H-bonded to E582. This pattern of interactions is common to all cyclic nucleotides crystallized to date in the CNBD of HCN channels. Interestingly, cAMP and cGMP in the binding site assume different conformations, characterized by an orientation anti and syn, respectively, of the purine ring. The $\mathrm{NH}_{2}$ group of adenosine forms an $\mathrm{H}$-bond with the carbonyl oxygen of R632, and some hydrophobic interactions are formed between the aromatic ring and residues in the C-helix and the $\beta$-roll. The $\mathrm{NH}_{2}$ group of guanosine is engaged in $\mathrm{H}$-bond with $\mathrm{T} 592$, whereas the $\mathrm{N} 1$ nitrogen is connected to R632 (C-helix) through a water molecule. cGMP is the only nucleotide that binds in a syn orientation: the purine nucleotides cIMP, cPMP, and $2-\mathrm{NH}_{2}$-cPMP, as well as the pyrimidine cCMP and cUMP, all bind in an anti-arrangement (Ng et al., 2016).

As stated before, HCN subunits display different sensitivity to cAMP; however, the crystal structures of the intracellular region of $\mathrm{HCN} 1, \mathrm{HCN} 2$, and HNC4 with bound cAMP are almost superimposable. The major structural variation was observed in the loop between the $\beta 4$ and $\beta 5$ strands, a region that is part of the binding site of cyclic nucleotides: some residues establish contacts with the purine ring of cAMP, and in this region there are localized some sequence differences (Xu et al., 2010, 2012; Lolicato et al., 2011). Xu et al. $(2010,2012)$ studied the different sensitivity of HCN2 and HCN4 to cAMP (Table 1), suggesting the importance of two substitutions in the $\beta 4-\beta 5$ loop, M572 and S575 in HCN2, which become Thr and Ala 
TABLE 3

Structures of HCN channel C-terminal domain deposited in the Protein Data Bank (PDB) up to November 2016

\begin{tabular}{|c|c|c|c|}
\hline PDB Code & Isoform (Residues) & Ligand & References \\
\hline $1 \mathrm{Q} 43$ & mHCN2 (443-640) & cAMP & Zagotta et al. (2003) \\
\hline 1QE3 & mHCN2 (443-645) & cGMP & Zagotta et al. (2003) \\
\hline $1 \mathrm{Q} 5 \mathrm{O}$ & mHCN2 (443-645) & cAMP & Zagotta et al. (2003) \\
\hline 2PTM & SpHCN (470-665) & cAMP & Flynn et al. (2007) \\
\hline 3BPZ & mHCN2 $(443-640)^{a}$ & cAMP & Craven et al. (2008) \\
\hline 3ETQ & mHCN2 $(443-640)^{b}$ & cAMP & Taraska et al. (2009) \\
\hline $3 \mathrm{FFQ}$ & mHCN2 & $\mathrm{Br}^{-}$ & Taraska et al. (2009) \\
\hline $3 \mathrm{OTF}$ & hHCN4 (521-739) & cAMP & $\mathrm{Xu}$ et al. (2010) \\
\hline $3 \mathrm{UOZ}$ & HCN1 (390-592) & cAMP & Lolicato et al. (2011) \\
\hline 3U10 & mHCN2 (470-672) & cAMP & Lolicato et al. (2011) \\
\hline 3U11 & hHCN4 (521-723) & cAMP & Lolicato et al. (2011) \\
\hline $4 \mathrm{EQF}$ & mHCN2 (857-863) & Trip8b (206-567) & Bankston et al. (2012) \\
\hline $4 \mathrm{HBN}$ & hHCN4 $(521-724)^{c}$ & cAMP & $\mathrm{Xu}$ et al. (2012) \\
\hline $4 \mathrm{NVP}$ & hHCN4 & 7CH-cAMP & Möller et al. (2014) \\
\hline $4 \mathrm{KL} 1$ & hHCN4 (521-723) & cGMP & Lolicato et al. (2014) \\
\hline $5 \mathrm{KHG}$ & mHCN2 (443-643) & $\mathrm{cCMP}$ & $\mathrm{Ng}$ et al. (2016) \\
\hline $5 \mathrm{KHH}$ & mHCN2 (443-643) & cIMP & Ng et al. (2016) \\
\hline $5 \mathrm{KHI}$ & mHCN2 (443-643) & cPMP & $\mathrm{Ng}$ et al. (2016) \\
\hline $5 \mathrm{KHJ}$ & mHCN2 (443-643) & cUMP & $\mathrm{Ng}$ et al. (2016) \\
\hline $5 \mathrm{KHK}$ & mHCN2 (443-643) & $2-\mathrm{NH}_{2}-\mathrm{cPMP}$ & Ng et al. (2016) \\
\hline $2 \mathrm{MNG}$ & HCN4 & - & Akimoto et al. (2014) \\
\hline $2 \mathrm{MPF}$ & HCN2 & - & Saponaro et al. (2014) \\
\hline
\end{tabular}

Mutations: ${ }^{a} \mathrm{E} 502 \mathrm{~K} ;{ }^{b} \mathrm{C} 508 \mathrm{~N}, \mathrm{C} 584 \mathrm{~S}, \mathrm{C} 601 \mathrm{~S} ;{ }^{c} \mathrm{~S} 672 \mathrm{R}$.

in HCN4. Interestingly, NMR-based evidence suggests an interaction with M572 of the flavonoid fisetin, the only compound structurally unrelated to cyclic nucleotides, which has been reported to activate mHCN2 expressed in Xenopus oocytes, with half-intrinsic activity with respect to cAMP (Carlson et al., 2013).

To understand the conformational rearrangement induced by the binding of cyclic nucleotides, it would be interesting to compare the holo (i.e., cAMP bound) and the apo (i.e., cAMP-free) forms. Unfortunately, nearly all the X-ray structures of the CNBD solved to date are in the holo form and only one apo structure has been obtained for HCN2 (Table 3) (Taraska et al., 2009). The bound and free HCN2 structures show high similarity, possibly due to the presence, in the apo form, of a bromide ion, in a position equivalent to the phosphate group of cAMP in the holo structure, which could induce the same conformational arrangement as the negatively charged cyclic nucleotide. However, comparison of the two structures has revealed a partial uncoiling of $\mathrm{F}^{\prime}$ helix and of the $\mathrm{C}$-terminal portion of the C-helix, and transition metal ion fluorescence resonance energy transfer has confirmed that the movements triggered by cAMP binding involve this part of the protein (Taraska et al., 2009). A NMR spectroscopy study, performed on HCN4 holo and apo forms, revealed that the conformational changes involve a larger portion, which includes, on one side, helices $\mathrm{E}^{\prime}-\mathrm{F}^{\prime}$-A (i.e., the N3A motif) surrounding the CNBD, and helices B and C (C-linker) on the other (Akimoto et al., 2014).

Very recently, the structure of $\mathrm{hHCN} 1$ has been determined at a $3.5 \AA$ resolution by means of cryoelectron microscopy, with and without cAMP bound (Lee and MacKinnon, 2017), allowing for the first time to compare holo and apo forms under static conditions: the clear change in the position of $\mathrm{A}, \mathrm{B}$, and $\mathrm{C}$ helices confirms what was found under dynamic conditions. This structure gave a picture, for the first time, of the unique features of this protein, which explain some of its "funny" behavior, as described above (see section Genetic and Molecular Characteristics of the HCN Family).

The crystal structure of the C-terminal domain of HCN4 with cGMP unexpectedly revealed two bound molecules, one in the canonical binding site and the other in a site, called by the authors C-linker pocket located at the interface between the C-linker and the CNBD (Lolicato et al., 2014). This second binding site has been proposed to have an inhibitory effect on cAMP activation: in fact, site-directed mutagenesis studies suggested that this is the interaction site of cyclic dinucleotides, which have been found to have an inhibitory activity (see section Modulation by Cyclic Nucleotides). This finding can be attractive for two reasons: first, the C-linker pocket site is found only in HCN4; therefore, cyclic dinucleotides may represent a mechanism to regulate specifically this isoform. Second, it may be possible to find small molecules that interact with this site, which should be selective for HCN4, as it actually has been proposed (Lolicato et al., 2014) (see section Isoform-Selective HCN Blockers).

2. Structure-Activity Relationships of Cyclic Nucleotides and Analogs. The affinity for the CNBD of cyclic nucleotides has been reported in two different studies (Scott et al., 2007; Möller et al., 2014). In both cases, 8-[[2-[(fluoresceinyl thioureido)amino]ethyl]thio]adenosine- $3^{\prime}, 5^{\prime}$-cyclic monophosphate, which binds with high affinity to the intracellular region of $\mathrm{HCN}$ channels, was used as fluorescent ligand, and a series of cyclic nucleotides was screened for their ability to 
displace it from the CNBD. The two studies used different proteins and number of compounds, but reached similar conclusions. In the first one, the affinity of 20 ligands was determined on a dimeric construct, made with the rat HCN2 CNBD and the DNA binding domain of the cAMP receptor protein (Scott et al., 2007). In the more recent one, 47 analogs were screened on the C-terminal domain (C-linker and CNBD) of mHCN1, mHCN2, and hHCN4 channels, kept as monomers by means of a maltose-binding protein tag (Möller et al., 2014).

The 47 tested compounds did not display selectivity for the three isoforms, but structural modifications greatly affected affinity. Among the naturally occurring cyclic nucleotides, cAMP was, as expected, the most potent, whereas others (cGMP, cCMP, cIMP, cPMP, cUMP) interacted with lower affinity. The pivotal role of the ionic bond with R591 has been confirmed by the drop-in affinity found for the cAMP and cGMP analogs having the equatorial phosphate oxygen atom substituted with sulfur; a similar replacement on the axial oxygen atom, involved in $\mathrm{H}$-bond with $\mathrm{Thr}$, reduced affinity too, although to a lesser extent. The 2 '-hydroxyl group forms an $\mathrm{H}$-bond as donor with a key glutamate residue (Zhou and Siegelbaum, 2007); this interaction is necessary because methylation strongly reduced or abolished binding. Substitution in position 8 with $\mathrm{Br}$, fluorescein, or other groups gave compounds with improved affinity, indicating some space available for bulky substituents. On the contrary, modifications on the $\mathrm{NH}_{2}$ groups of cAMP and cGMP were detrimental, suggesting that these groups too are involved in binding. Unexpectedly, the replacement of the nitrogen atom in position 7 with $\mathrm{CH}$ on $\mathrm{CAMP}$, cGMP, and other analogs improved affinity: the $\mathrm{EC}_{50}$ for $7 \mathrm{CH}$-cAMP were 100 -, 64-, and 108-fold lower than those of cAMP on, respectively, HCN1, HCN2, and HCN4 C-terminal domains. The reason for this increase in affinity was clear from the X-ray structure of the complex of 7CH-cAMP with the HCN4 intracellular region; although cAMP and its $\mathrm{CH}$ analog interact in the same way, the hydrophobic environment surrounding the seven position of the purine ring stabilizes a lipophilic $\mathrm{CH}$ group better than a hydrophilic $\mathrm{N}$ atom. On human HCN4 channel, expressed in HEK293 cells, 7CH-cAMP behaves as an activator with potency four times higher than cAMP; moreover, it displayed some selectivity for HCN channels with respect to PKA and to exchange protein directly activated by cAMP.

\section{B. Pharmacology of HCN Blockade by Exogenous Ligands}

1. Ivabradine, Cilobradine, and Other Specific Bradycardic Agents. The definition "specific bradycardic agent" (SBA) refers to a group of structurally diverse substances, discovered in the second half of 1900 . These compounds belong to two different chemical classes:
$\mathrm{N}$-alkyl-aminoimidazolines, exemplified by alinidine (2-[N-allyl-N-(2,6-dichlorophenyl)amino]imidazoline) and phenylalkylamines structurally related to falipamil (AQ-A-39) (Fig. 6). The word "specific" meant that these molecules, and their congeners, were able to reduce heart rate at concentrations at which other hemodynamic parameters were not affected; it was proposed that these compounds exerted their activity directly on the SAN, blocking the funny current, just discovered at that time (Kobinger and Lillie, 1987). Later on, it was found that the activity of SBAs was not confined to cardiac tissue because these compounds could block the hyperpolarization-activated current also in neurons (Pape, 1994).

The development of alinidine and analogs was stopped due to the concern about the formation of clonidine as metabolite, and by the observation of visual side effects in phase I clinical trials (Kobinger, 1987). On the contrary, the research on phenylalkylamines yielded some interesting compounds. Increasing the size of the five-membered pyrrolidone ring of falipamil into a seven-membered benzazepinone moiety gave a more potent and safer compound, zatebradine (UL-FS49), which entered clinical trials; the development of this molecule too was halted due to adverse effects. The benzazepinone moiety is found also on other two phenylalkylamines: cilobradine (DH-AK269) and ivabradine (S16257). Both compounds are reduced-flexibility analogs of zatebradine: in cilobradine, the threemethylene chain became part of a six-membered piperidine ring, whereas in ivabradine the phenethyl group was incorporated into a bicyclo[4.2.0]octa-1(6),2,4-trien-7-yl methyl moiety. In both cases, a chiral center was introduced into the molecules, and the eutomer is the S enantiomer (Figure 6).

The three drugs have a similar mode of action: they reduce spontaneous AP firing in SAN preparation, mainly through a reduction in the rate of diastolic depolarization (Thollon et al., 1994). On sheep Purkinje fibers, these drugs reduced $I_{\mathrm{h}}$ amplitude, without modifying the voltage dependence (Van Bogaert and Pittoors, 2003). $I_{\mathrm{h}}$ blockade is use-dependent (Goethals et al., 1993) and occurs on the open channel (Goethals et al., 1993; DiFrancesco, 1994; Bois et al., 2007). No effect on contractility is produced (Lillie and Kobinger, 1986; Bois et al., 1996). Cilobradine is reported to be more potent than zatebradine in sheep Purkinjie fibers and mouse DRG (Raes et al., 1998). At variance with the other two drugs, zatebradine prolonged APs in some cardiac preparations (Thollon et al., 1994; Perez et al., 1995b), showing class III antiarrhythmic properties (Valenzuela et al., 1996). This could be one of the reasons for which the development of zatebradine has stopped; another was, again, the observation of visual side effects, coming from zatebradine blockade of $I_{\mathrm{h}}$ involved in the recovery of photoresponse in retinal rods (Satoh and Yamada, 2002). Site-directed mutagenesis, 


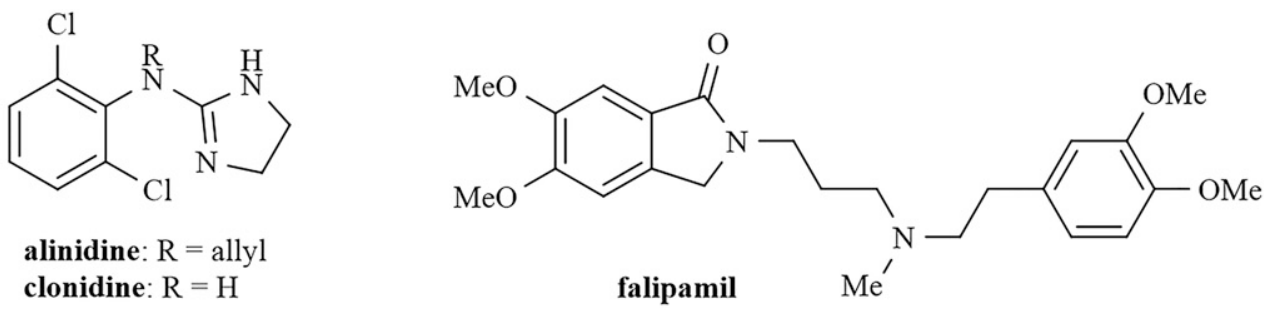

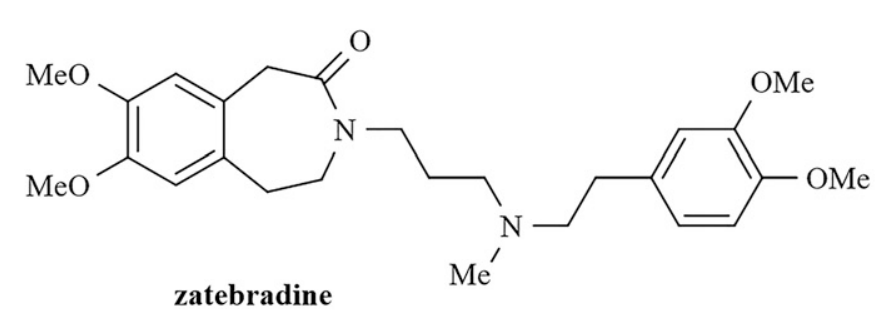

zatebradine

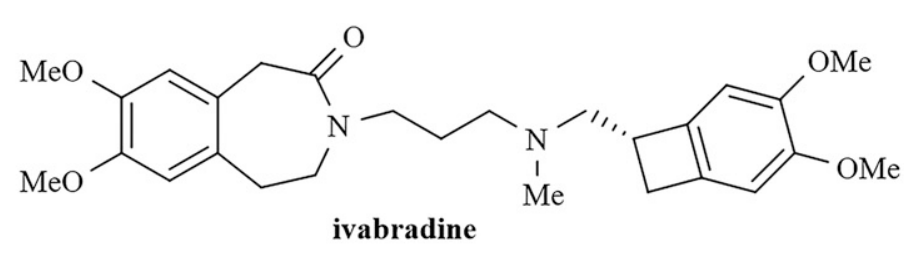

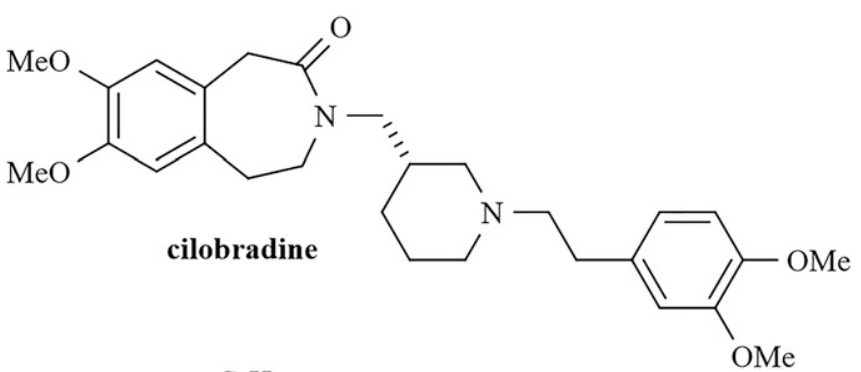<smiles></smiles>

Fig. 6. Chemical structure of specific bradycardic agents.

electrophysiology, and molecular modeling studies suggest that cilobradine and ivabradine share a common binding site, located in the internal mouth of the channel pore (Cheng et al., 2007; Bucchi et al., 2013); the proposed mechanism is that these drugs enter the binding site from the intracellular side of the channel (Bucchi et al., 2006).

ZD7288 (Fig. 6) is another blocker of the hyperpolarizationactivated current, which is structurally unrelated to the other SBAs. Its pharmacological characterization showed that it behaves as specific bradycardic agent acting on the SAN without having any other direct effects on the heart (BoSmith et al., 1993). Different from phenylalkylamines, the $I_{\mathrm{h}}$ blockade by ZD7288 is not use-dependent, but there is evidence from site-directed mutagenesis that the binding site is the same of ivabradine (Bucchi et al., 2013). ZD7288 has not been developed as drug, but it is widely used as a tool to study $I_{\mathrm{h}}$.

The word "specific" does not imply that SBAs are completely selective for HCN channels; all compounds reported in this section are able to interact also with other targets. For instance, alinidine is a $\mathrm{K}^{+}$channel blocker (McPherson and Angus, 1989; Jonas et al., 1992); ZD7288 interacts with $\mathrm{Na}^{+}$and $\mathrm{Ca}^{2+}$ channels (Sanchez-Alonso et al., 2008; Wu et al., 2012). Several papers have reported that the interaction of ivabradine with other ion channels occurs at concentration higher than those active on HCN channels (Perez et al., 1995a; Bois et al., 1996; Delpon et al., 1996; Koncz et al., 2011); however, more recently, two independent studies showed that ivabradine blocks human ether a-go-go (hERG) $\mathrm{K}^{+}$ channels and HCN4 channels with similar potency (Lees-Miller et al., 2015; Melgari et al., 2015), raising concern on the safety of this drug (Hancox et al., 2015).

The SBAs discussed in this section were disclosed before discovering the heterogeneity of HCN channel family; their ability to discriminate among HCN channel isoforms has been checked only for zatebradine, cilobradine, and ivabradine (see next section).

2. Isoform-Selective HCN Blockers. The ability of ivabradine, cilobradine, and zatebradine to discriminate among HCN channel isoforms has been tested on human homomeric HCN1-4 channels expressed in HEK293 cells (Stieber et al., 2006). The three compounds did not display selectivity or preference for one isoform over the others; the $\mathrm{IC}_{50}$ were in all cases in the low micromolar range. The lack of selectivity may not surprise, if one considers that the four isoforms share a high degree $(80 \%-90 \%)$ of sequence identity in the region comprising the six transmembrane segments (S1-S6) and the CNBD (Kaupp and Seifert, 2001); this region harbors the proposed binding site for ivabradine, cilobradine, and ZD7288. However, sequence alignment of the S5-P-S6 region of HCN1, HCN2, and HCN4 isoforms-where site-directed mutagenesis studies locate the residues important for binding (Shin et al., 2001; Cheng et al., 2007; Chan et al., 2009; Bucchi et al., 2013)—reveals some differences in the sequence, that, in principle, could pave the way to selectivity for compounds' interaction at this site.

Indeed, structural modifications of the nonselective blocker zatebradine gave compounds (Fig. 7) showing 


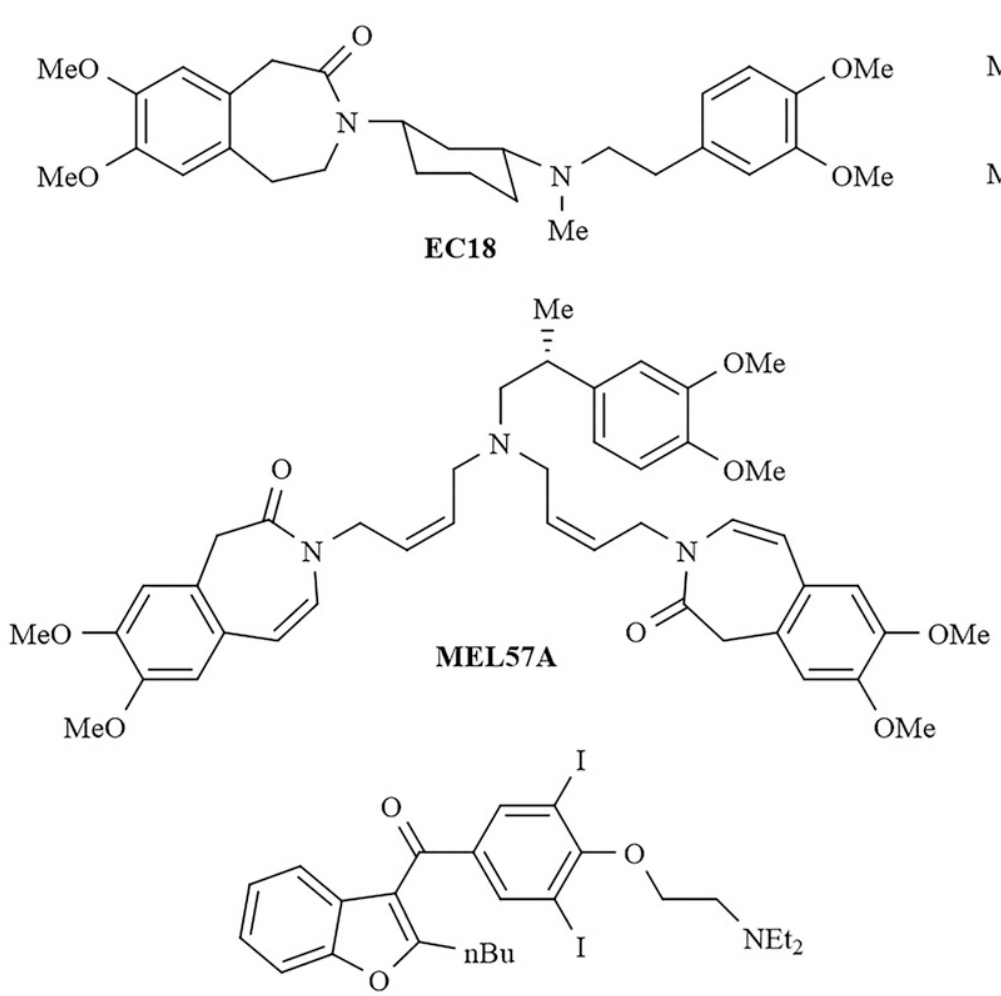

Amiodarone<smiles>CCCCCCCCCCCCCCCCN1C=Cc2cc(OC)c(OC)cc2CC1=O</smiles><smiles>CCOc1ccccc1C(=O)NCC1(N2CCNCC2)CCCCC1</smiles><smiles>N#Cc1cccc(CN2CCC(N(Cc3cccnc3)C(=O)Nc3ccccc3-c3ccccc3)CC2)c1</smiles>

Fig. 7. Chemical structure of isoform-selective HCN blockers.

different activity on homomeric HCN1, HCN2, and HCN4 channels expressed in HEK293 cells. The inclusion of the three-methylene chain of zatebradine into a cyclohexane ring gave EC18, showing a sixfold preference for HCN4 over HCN1 and HCN2 channels (Del Lungo et al., 2012). The replacement of the threemethylene chain with a cis-butene moiety, combined with the introduction of a chiral center on the phenethyl group, gave MEL55A [labeled in the original paper as (R)-5], which was fourfold and 11-fold more potent on HCN2 than on $\mathrm{HCN} 1$ and $\mathrm{HCN} 4$, respectively (Melchiorre et al., 2010). MEL57A, serendipitously discovered while synthesizing MEL55A, displayed 30- and 170-fold selectivity for HCN1 over HCN2 and HCN4, respectively. Notably, the selectivity found in recombinant systems was maintained in tissues expressing different HCN isoforms. On guinea pig SAN cells, the reduction of $I_{\mathrm{h}}$ produced by EC18 was threefold higher than that induced by MEL57A; both compounds were tested at concentrations close to the $\mathrm{IC}_{50}$ values. In contrast, in mouse DRG neurons, MEL57A, but not EC18, significantly reduced $I_{\mathrm{h}}$. In dog cardiac Purkinje fibers, EC18 reduced the amplitude and slowed the slope of the spontaneous diastolic depolarization, whereas MEL57A was ineffective (Del Lungo et al., 2012). In cultured DRG, MEL55A reduced $I_{\mathrm{h}}$ amplitude and cell excitability, its effect being quantitatively similar to that of ivabradine (unpublished data). The utility of isoformselective and HCN-channel-specific compounds appears evident in studying $I_{\mathrm{h}}$ from tissues expressing different isoforms: for instance, the application of the HCN4preferring blocker EC18 suggested a functional role of this isoform in mediating $I_{\mathrm{h}}$ in GABAergic interneurons of the dorsal part of the lateral geniculate nucleus (Leist et al., 2016).

Researchers at Johnson \& Johnson (San Diego, CA) disclosed a HCN1-selective compound. Starting from the high throughput screening of a proprietary library, and after optimization of the initial lead, piperazine 1 was obtained (Fig. 7); this compound was 12-, 7-, and 10-fold more potent on homomeric HCN1 channels than on HCN2, HCN3, and HCN4, respectively (McClure et al., 2011). Differently from the nonselective compound ZD7288, 1 displayed antihyperalgesic activity in a spared nerve injury model at doses at which heart rate was not affected.

Among the compounds described in the next section, amiodarone shows some isoform selectivity: its $\mathrm{IC}_{50}$ value for blockade of homomeric channels expressed in Xenopus oocytes is 4- and 22-fold lower on HCN4 than on HCN1 and HCN2, respectively (Fan et al., 2011). However, multi-ion channels blocking properties may obscure the selectivity of this compound.

Although it is reasonable to hypothesize that EC18, MEL55A, and MEL57A, being structural analogs of 
ivabradine and cilobradine, bind to the same interaction site, nothing is known about the binding site of compound 1 and amiodarone. As reported in the previous section (Lessons from Crystallography), the X-ray structure of the complex cGMP-HCN4 revealed the existence of a pocket in the C-linker, proposed to be the binding site for cyclic dinucleotides, but available also for synthetic modulators (Lolicato et al., 2014). Molecules binding at this site should display selectivity because this pocket is not present in other isoforms. Indeed, by means of virtual screening, a compound has been disclosed (N'-biphenyl-2-yl-N-[1-(3-cyanobenzyl)piperidin-4yl]-N-(pyridin-3-ylmethyl)urea, 2; Fig. 7), which was found able to antagonize cAMP activation on HCN4, but not on HCN2 channels. This pocket may represent a new opportunity for delivering HCN4-selective compounds.

3. HCN Blockade by Other Drugs. Several drugs have been shown to block HCN channel, a property that can either contribute to their pharmacological activity or, alternatively, produce side effects. Some of the compounds mentioned in this section are $\mathrm{G}$ protein-coupled receptor modulators, controlling cAMP concentration through adenylyl cyclase-coupled $\mathrm{G}_{\alpha}$ proteins; therefore, they could be endowed with mixed direct-indirect activity. In the following discussion, only compounds used in the clinic are included; for a more comprehensive list, see Romanelli et al. (2016). The compounds have been divided into subgroups according to their pharmacological activity.

a. Analgesic/Antihyperalgesic drugs. Dexmedetomidine and clonidine are $\alpha_{2}$-AR agonists commonly used for several indications, including analgesia. They share some structural similarities, carrying a five-membered imidazole or imidazoline ring. In addition, as discussed in section Ivabradine, Cilobradine, and Other Specific Bradycardic Agents, clonidine is a metabolite of the SBA alinidine. Both compounds have been shown to block recombinant HCN channels expressed in HEK293 cells (Knaus et al., 2007; Yang et al., 2014), and in vivo studies suggested that the analgesia by dexmedetomidine can be produced by an $\alpha 2$-independent mechanism (Brummett et al., 2011; Yang et al., 2014). For both drugs, bradycardia is a common side effect (Anger, 2013; Isbister et al., 2017), although only clonidine has been tested on SAN (Knaus et al., 2007). The analgesic activity of both compounds can also be due to a receptor-mediated mechanism: $\alpha_{2}$-AR activation by these drugs is reported to decrease $I_{\mathrm{h}}$ in several neuronal preparations (Parkis and Berger, 1997; Carr et al., 2007; Shirasaka et al., 2007; Inyushin et al., 2010), including some involved in pain perception (Yagi and Sumino, 1998; Takeda et al., 2002). In some instances, it appears that the receptor-mediated inhibition of HCN channels does not occur through the classic $\mathrm{G}_{\alpha, \mathrm{i}}$-linked reduction of cAMP concentration, rather through activation of protein kinase $C$ via $G_{\beta \gamma}$ modulation (Carr et al., 2007; Inyushin et al., 2010).
Loperamide and tramadol are opioid receptor agonists, which have been reported to block $I_{\mathrm{h}}$ by opioid receptor-independent mechanisms. Tramadol was tested on GH3 cells, where it inhibited $I_{\mathrm{h}}$ with micromolar potency; the effect was not affected by preincubation with naloxone (Liu et al., 2009). Loperamide is mainly used as antidiarrheal drug, but it showed analgesic activity and antihyperalgesic properties in animal models of neuropathic pain; in some instances, this activity was not dependent of opioid receptors (Ringkamp et al., 2012). Loperamide was able to block HCN1 and HCN4 channels expressed in HEK293 cells (Vasilyev et al., 2009), and to inhibit $I_{\mathrm{h}}$ in rat DRG, acting from the extracellular side of the channel (Vasilyev et al., 2007); the authors suggested that this effect could contribute to the analgesic activity of the drug.

The antihyperalgesic activity of minocycline has been tested in several models of neuropathic pain, and it has been associated with inhibition of microglia activation (Mika, 2008; Möller et al., 2016); the molecular mechanism is unknown, but could be related also to the ion channel-blocking properties of this drug. On substantia gelatinosa neurons of rat spinal cord, minocycline reduced $I_{\mathrm{h}}$ and decreased the rate of AP firing; the maximal inhibition was $40 \%$ and was produced by a direct interaction with the channel from the extracellular side (Liu et al., 2015). Potency was in the micromolar range, but $\mathrm{IC}_{50}$ value was two orders of magnitude higher than that for $\mathrm{Na}^{+}$channel blockade in DRG (Kim et al., 2011).

Eugenol, a dental analgesic obtained from clove oil, was found able to block hyperpolarization-activated current and decrease firing of APs in trigeminal ganglion neurons. After rat infraorbital nerve chronic constriction injury, it reversed mechanical allodynia at lower doses with respect to those active in thermal hyperalgesia. The $I_{\mathrm{h}}$-blocking properties may contribute to the analgesic and antihyperalgesic activity of this molecule (Yeon et al., 2011).

$b$. General anesthetics. Propofol is a general anesthetic, with additional antiemetic and antiepileptic properties (Kotani et al., 2008; Lundström et al., 2010). Propofol is a small lipophilic molecule, which interacts with multiple targets, including several ion channels (Kojima et al., 2015). $I_{\mathrm{h}}$ blockade by propofol has been studied in several tissues: hippocampal neurons (Funahashi et al., 2001; Higuchi et al., 2003), area postrema (Funahashi et al., 2004), thalamocortical neurons (Ying et al., 2006; Chen et al., 2009a), and EC (Li et al., 2016). Overall, these studies suggest that $I_{\mathrm{h}}$ blockade can contribute substantially to the pharmacological actions of the drug, which seems to involve mainly HCN1 and HCN2 channel isoforms (Ying et al., 2006; Chen et al., 2009a). In X. laevis oocytes expressing homomeric $\mathrm{HCN} 1, \mathrm{HCN} 2$, and HCN4, and heteromeric HCN1-HCN2 channels, HCN1 was the 
most sensitive isoform (Cacheaux et al., 2005; Chen et al., 2005). Consistent with HCN channel blockade are the findings that propofol reduces mechanical and thermal hyperalgesia in a peripheral nerve ligation model of neuropathic pain (Tibbs et al., 2013), and that bradycardia is a common side effect after administration of this drug (Lundström et al., 2010).

Ketamine is another general anesthetic with multiple action sites, among which HCN channels: in HEK293 cells the drug strongly inhibited homomeric HCN1 and heteromeric HCN1-HCN2 channels, but not homomeric HCN2 channels. This activity is likely to contribute to the hypnotic action of ketamine, as demonstrated in HCN1-KO mice (Chen et al., 2009a). Interestingly HCN1 inhibition alone can theoretically explain the changes in EEG produced by ketamine or propofol, without taking into account interaction with NMDA or GABA receptors (Bojak et al., 2013).

Other volatile general anesthetics have been shown to reduce $I_{\mathrm{h}}$; for instance, halotane and isoflurane interact with HCN1 and HCN2 channels, producing a hyperpolarizing shift in voltage dependence of activation and a decrease in maximal available current at clinically relevant concentrations (Chen et al., 2005, 2009b). Studies using global and forebrain-selective HCN1-KO mice showed that interaction with this isoform in the forebrain contributes to hypnotic and amnestic effects of isoflurane and sevoflurane, but not to their immobilizing action (Zhou et al., 2015b). In thalamocortical neurons, xenon reduced signal propagation by interacting with HCN2 channels, the predominant isoform expressed in this tissue (Ludwig et al., 1998); this effect was not seen in HCN2-KO animals. Accordingly, xenon produced a sedative effect in WT mice, but not in mice lacking HCN2 channels (Mattusch et al., 2015).

c. Local anesthetic and antiarrhythmic drugs. The $I_{\mathrm{h}}$-blocking activity of the local anesthetic and antiarrhythmic drug lidocaine has been measured in recombinant systems and on neuronal and cardiac tissues. Lidocaine was tested on homomeric mHCN1, mHCN2, and mHCN4, and heteromeric mHCN1HCN2 channels expressed in $X$. laevis oocytes and HEK293 cells (Meng et al., 2011): on all isoforms the drug caused a decrease in both tonic and maximal current and slowed current activation kinetics; only for HCN1-containing channels it produced a hyperpolarizing shift in voltage dependence of activation. On rat DRG (Bischoff et al., 2003; Putrenko and Schwarz, 2011) and in substantia gelatinosa neurons (Hu et al., 2016), the blockade was reversible; the doseresponse curves yielded similar $\mathrm{IC}_{50}$ values $(\sim 80$ $99 \mu \mathrm{M})$. In a mice model of sciatic nerve block, lidocaine was more potent in WT mice that in $\mathrm{HCN}^{-1-}$ mice, and the duration of lidocaine anesthesia was longer in WT than in $\mathrm{HCN}^{-1-}$ mice (Zhou et al., 2015a). It was suggested that HCN channel blockade might substantially contribute to the drug action during epidural and spinal anesthesia. When tested on rat ventrobasal thalamocortical relay neurons, lidocaine blocked $I_{\mathrm{h}}$ with potency similar to that found in DRG (Putrenko and Schwarz, 2011); the authors suggest that this activity could contribute to lidocaine's systemic analgesic actions and may partially explain neurotoxicity.

In addition to lidocaine, bupivacaine and mepivacaine also have been tested on rat DRG neurons (Bischoff et al., 2003). The mechanism of $I_{\mathrm{h}}$ blockade was similar to that of lidocaine; bupivacaine was about twice and four times as potent as lidocaine and mepivacaine, respectively.

In cardiac tissues, the $I_{\mathrm{h}}$-blocking properties of lidocaine have been demonstrated more than 30 years ago in sheep cardiac Purkinje fibers (Carmeliet and Saikawa, 1982), and more recently on rabbit SAN myocytes (Rocchetti et al., 1999). In the latter study, the $\mathrm{IC}_{50}$ value (38 $\mu \mathrm{M}$, twofold lower than in neuronal tissues) suggested some concern on a possible interference of the drug on normal cardiac automaticity. Indeed, when tested on recombinant $\mathrm{mHCN} 4$ channel, $I_{\mathrm{h}}$ blockade was found significant also at physiologic potentials $(-70 \mathrm{mV})$ (Meng et al., 2011).

However, under different experimental conditions, the effect produced by lidocaine on recombinant rabbit HCN4 channels, at $-70 \mathrm{mV}$, was considered clinically not relevant (Tamura et al., 2009). This study was performed on a large series of antiarrhythmic drugs, to gain information about their effects on the pacemaker current for a more rational clinical use of these compounds. To simulate physiologic conditions, i.e., the tonic stimulation by the sympathetic nerve and the presence in the cytosol of cyclic nucleotides, the blocking activity was measured in presence of $0.3 \mathrm{mM}$ intracellular cAMP, and the calculated $\mathrm{IC}_{50}$ values were compared with therapeutic concentrations. For several compounds (quinidine, disopyramide, cibenzoline, mexiletine, aprindine, propafenone, flecainide, propranolol, verapamil, sotalol, as well as lidocaine), the effect was considered negligible; on the contrary, the $\mathrm{IC}_{50}$ values of amiodarone and bepridil were close to their therapeutic concentrations.

The effect of amiodarone on $I_{\mathrm{h}}$ was further characterized on human HCN channel isoforms (HCN1, HCN2, and HCN4) expressed in X. laevis oocytes (Fan et al., 2011): on all isoforms the blockade was concentrationand use-dependent, but the activation curves were not modified. Interestingly, amiodarone was found to be 4 and 20 times more potent on HCN4 that on HCN2 and HCN1, respectively. Amiodarone was found to block $I_{\mathrm{h}}$ also in rabbit SAN preparations (Satoh, 1991), ventricular myocytes from spontaneously hypertensive and normal rats ( $\mathrm{Li}$ et al., 2014a), but not in isolated human atrial myocytes (Hoppe and Beuckelmann, 1998). In ventricular myocytes from spontaneously hypertensive rats, amiodarone significantly suppressed $I_{\mathrm{h}}$ density 
and downregulated HCN2 and HCN4 expression (Li et al., 2014a).

Dronedarone is a structural analog of amiodarone, used in the treatment of AF. On hHCN4 channel expressed in Chinese hamster ovary cells, dronedarone was equipotent with amiodarone and ivabradine in blocking $I_{\mathrm{h}}$ (Bogdan et al., 2011). Clinical trials have evidenced a mild bradycardic effect of this drug (Singh et al., 2007), which has been shown to be mainly due to HCN channel inhibition (Sobrado et al., 2013). In a porcine model, dronedarone induced a significant reduction in ventricular rate during $\mathrm{AF}$, owing to $I_{\mathrm{h}}$ inhibition in AVN, which slowed conduction (Verrier et al., 2014).

\section{In Vivo Studies and Clinical Pharmacology}

1. Ivabradine in Angina and Heart Failure. The current therapeutic indication for ivabradine stems from its primary development as selective HCN blocker: ivabradine is the only commercially available specific bradycardic agent. To understand why bradycardia represents an attractive target in angina, it is worth to recall briefly the mechanisms underlying myocardial ischemia. Ischemic cardiomyopathy arises from an imbalance between oxygen supply to and demand by the myocardium. Several factors contribute to this disequilibrium, namely flow reduction through epicardial coronary arteries, due to plaques, vasospasm, or-as more recently acquired-microcirculatory dysfunction (for a review, see Camici et al., 2016). Myocardial perfusion and oxygen extraction occur mainly during the diastolic phase, in either healthy or diseased heart, but allowing a sufficient rest time between two consecutive beats is more crucial for the latter condition, due to the uneven blood flow. At the same time, heart rate is the primary determinant of oxygen demand. Therefore, decreasing heart rate is a primary therapeutic goal in ischemic cardiomyopathy, traditionally achieved by using betablockers, which attenuate angina symptoms. Nevertheless, a significant percentage of patients do not achieve the optimal beta-blocker dosage because of adverse effects or contraindications. Thus, recent European Society of Cardiology guidelines recommend ivabradine as a second-line therapy (class IIa, level B) in patients with ischemic cardiomyopathy intolerant to or poorly controlled by beta-blockers (Montalescot et al., 2013). This recommendation is based on clinical evidence: in the BEAUTIFUL trial, ivabradine reduced severe cardiovascular events in patients with angina and a heart rate $\geq 70$ beats per minute (bpm) (Fox et al., 2008). Alone or combined with beta-blockers, ivabradine reduces SAN rhythm, both at rest and during exercise, without (further) depressing contractility.

However, recent work suggests that the interplay between heart rate and cardiac protection might be more complex than a plain cardiac cycle/oxygen balance scheme. In animal models of ischemic cardiomyopathy, ivabradine protects against risk factors, such as atherosclerotic plaque formation (Custodis et al., 2008), energetic imbalance, and electrophysiological remodeling (Ceconi et al., 2011). These results led to new hypothesis on the relationship between heart rate reduction and improved coronary perfusion, because of collateral artery growth and/or recovery of endothelial cell function during bradycardia (Schirmer et al., 2012). Thus, the distinction between classic and pleiotropic (ancillary) mechanisms of action is tenue, becausebased on present knowledge-all can be largely ascribed to HCN blockade in SAN cells. Indeed, at variance with beta-blockers, ivabradine does not possess intrinsic vasoconstrictive activity, which may explain a gain of function during exercise, i.e., by preserving coronary dilation mediated by catecholamines (Camici et al., 2016). In the same line, the reduction of heart rate, but not the dosage, was significantly related to the survival in patients with heart failure treated with beta-blockers (McAlister et al., 2009).

In the SHIFT Trial (Bohm et al., 2010), adding ivabradine to current standard therapy in patients with heart failure safely lowered heart rate below $70 \mathrm{bpm}$, which is considered a desirable target to improve outcome in patients with ventricular dysfunction not often achieved with beta-blockers due to side effects. More recently, the SIGNIFY study in patients with preserved ejection fraction and coronary artery disease, in which ivabradine was added to standard background therapy, showed a significant increase in cardiovascular events concomitant to, or despite, heart rate reduction (about $60 \mathrm{bpm}$ versus $70 \mathrm{bpm}$ ) (Fox et al., 2014). Based on the results of these three large studies and their post hoc analysis, ivabradine should be used as a second-line therapy to reduce the risk of hospitalization in patients with symptomatic chronic heart failure and resting heart rate $>70 \mathrm{bpm}$ despite maximally tolerated dosage of beta-blockers (or contraindications to them) (Ponikowski et al., 2016). Adverse effects reported in these large trials also reflect ivabradine cardiac and extracardiac actions: the most common are bradycardia and visual disturbances (phosphenes) due to blockade of - respectively—cardiac and retinal HCN channels.

a. HCN blockade and arrhythmogenesis: an open issue. Far less defined and consequently exploitable are the potential antiarrhythmic profile of ivabradine and in general HCN blockade. Verrier et al. (2013) showed that the capability to control ventricular rate by dronedarone, the de-iodinated analogous of amiodarone, resides in its HCN-blocking effect on AVN conduction in a pig model of AF. This is not surprising because, as described above, HCN4 channels are functionally relevant in nodal conduction (Liu et al., 2008). Subsequent work by the same group reported a similar effect in pigs treated with ivabradine alone (Verrier et al., 2014) or with ranolazine (Verrier et al., 2015): the 
association reduced AVN conduction and AF dominant, leaving ventricular contractility and duration of the QT interval (an ECG marker of repolarization velocity) unaltered. Finally, recent reports in AF patients showed improved rate control with ivabradine added to beta-blockade (Caminiti et al., 2016; Kosiuk et al., 2016), although these sporadic observations are obviously too weak for drawing conclusions.

As mentioned above, a subgroup analysis of the SIGNIFY study unraveled increased cardiac events in patients with preserved contractile function treated with ivabradine, but neither bradycardia nor emergent $\mathrm{AF}$ appear to have an impact (Fox et al., 2015). A proarrhythmic potential of HCN blockade has been inferred based on telemetric recording of ECGs in mice treated with different specific bradycardic agents (Stieber et al., 2004). Periodic fluctuations of the R-R interval appeared in the ECG, depending on dosage and drug type, the order of potency being cilobradine $>$ zatebradine $>>$ ivabradine, without significant alterations in AVN conduction. These fluctuations resemble a well-known phenomenon termed Wenckebach's periodicity, typical of the AVN. In SAN cells, the mechanism underlying the sinoatrial Wenckebach's periodicity may reside in the different HCN expression and sensitivity to bradycardic agents in the central versus peripheral sinus node (Stieber et al., 2004; Mangoni and Nargeot, 2008). Finally, the bradycardic effect of ivabradine might stimulate the sympathetic drive, which is per se a proarrhythmic stimulus (Dias da Silva et al., 2015).

However, extrapolation of data obtained in rodents to the clinical setting may be unwarranted, and indeed the usefulness of ivabradine has been postulated in $\mathrm{AF}$, although limited clinical evidence exists at present (Turley et al., 2016). Of note, $I_{\mathrm{h}}$ seems to undergo significant remodeling in chronic $\mathrm{AF}$, as described above (Stillitano et al., 2013). Such a gain of function in the working atrial myocardium parallels that observed by several groups, including some of us, in hypertrophied and failing ventricular cardiomyocytes [reviewed in Sartiani et al. (2015)]. It is interesting to observe that chronic treatment with ivabradine exerts a reverse remodeling action in animal models of heart failure (Ceconi et al., 2011), by counteracting electrophysiological abnormalities, including HCN overexpression and function in atrial and ventricular cardiomyocytes (Suffredini et al., 2012). Finally, off-label uses of ivabradine have been reported in other settings (Oliphant et al., 2016), although evidence from clinical trials is lacking.

\section{Treatment of Pain.}

a. HCN channel modulation in neuropathic pain. The analgesic action of the HCN channel blocker ZD7288 in different models of neuropathic pain has been largely demonstrated (Dalle and Eisenach, 2005; Jiang et al., 2008; Takasu et al., 2010; Nava et al., 2014). In particular, tactile allodynia and thermal hyperalgesia were largely decreased by ZD7288 application. This is not surprising due to the wide expression of $\mathrm{HCN}$ channels along the peripheral pain neuraxis (Papp et al., 2006; Papp et al., 2010). Interesting results derived from genetically engineered $\mathrm{HCN}$ mutants underlined the important role played by HCN2 in neuropathic pain maintenance (Schnorr et al., 2014). Emery et al. (2011, 2012) demonstrated that conditional deletion of HCN2 abolished pain behavior in the chronic constriction injury model. These results were largely confirmed in a different model of neuropathy using a virtually identical HCN2 mutant (snsHCN2KO) (Herrmann et al., 2015), thus pointing out $\mathrm{HCN} 2$ as the most important subunit modulating neuropathic pain.

However, in chemotherapy-induced periphery neuropathic models, HCN1 modification seems to play a prominent role compared with HCN2 in modulating pain sensitization. Recently, Zhang and Dougherty (2014) demonstrated an increased expression of HCN1 in DRG neurons in a rat model of paclitaxel-induced peripheral neuropathy. They also observed an increased frequency of spontaneous discharging neurons in both myelinated and unmyelinated neurons. The hyperpolarization-activated current mediated by HCN1 may facilitate the rise of spontaneous activity in these neurons (Zhang and Dougherty, 2014). Furthermore, Descoeur et al. (2011) observed a significant increase in HCN1 mRNA levels in DRG from oxaliplatin-treated mice. Moreover, they observed that ivabradine exerted analgesic effect on cold allodynia, but not on mechanical hyperalgesia. These two examples provide evidence of a prominent role for HCN1 channel in chemotherapy-induced neuropathy.

Recently, the role of $\mathrm{HCN}$ channels in CNS for neuropathic pain has also been investigated. Interestingly, sciatic nerve injury caused an activity-dependent dysfunction of HCN channels in the dendrites of layer 5 pyramidal neurons, resulting in an increased neuronal activity in cortical areas, which has been postulated to be one of the key manifestations of chronic pain (Kuner, 2010; Saab, 2012). It was demonstrated that specific activation of $5-\mathrm{HT}_{7}$ receptor increased $\mathrm{HCN}$ channel function, thus restoring normal dendritic integration and reducing mechanical pain hypersensitivity in nerve-injured animals in vivo (Santello and Nevian, 2015).

b. Participation of HCN channels in inflammatory pain behavior. HCN appears to play a role also in modulation of inflammatory pain. Both nonselective $I_{\mathrm{h}}$ blockers and genetic deletion of HCN display a significant analgesic effect in different animal models of inflammation (Dunlop et al., 2009; Emery et al., 2011). The cAMP-sensitive isoform HCN2 seems to be particularly involved in heat hyperalgesia, as inferred from experiments using conditional HCN2-KO mice challenged with carrageenan or prostaglandin E2-mediated inflammatory stimuli (Emery et al., 2011). The inflammatory processes, 
which activate signaling transduction pathways, cause an increase of cAMP in pain-sensing neurons responsible for peripheral HCN2 channel activation, which in turn controls the acute phase of neuronal hypersensitivity. Indeed, cAMP shifts the resting membrane potential to depolarized values and increases AP firing in isolated DRG neurons, whereas these modifications do not occur in HCN2 KO or by using nonselective HCN channel blockers (Emery et al., 2011; Schnorr et al., 2014; Young et al., 2014). On the contrary, models of inflammation not implying $\mathrm{G}_{\mathrm{s}}$ activation are insensitive to $\mathrm{HCN} 2$ deletion or HCN channel blockade (Schnorr et al., 2014), thus demonstrating the absence of HCN influence on cAMPindependent nociceptive behavior.

Similarly, chronic inflammatory states, in which transcriptional regulatory processes are more important compared with intracellular cAMP increase for HCN-mediated sensitization, have been observed to be partially HCN-independent. Indeed, it appears that HCN channels only modulate tactile hypersensitivity, being heat hyperalgesia not affected by either HCN2 deletion or HCN channel block in chronic inflammatory models (Luo et al., 2007; Weng et al., 2012; Schnorr et al., 2014). These results suggest that distinct neuronal pathways convey mechanical and thermal sensitization in chronic inflammatory conditions (Abrahamsen et al., 2008; Cavanaugh et al., 2009; Herrmann et al., 2015). Nonetheless, pharmacological targets modulating $\mathrm{G}_{\mathrm{i}}$-coupled receptors have been reported to interact via $\mathrm{HCN}$ modulation to a decreased nociceptive response (Resta et al., 2016).

\section{Conclusions}

Despite the mass of information that has been accumulating on the biophysical and molecular properties of HCN channels and their (patho)physiologic role in excitable (and not-excitable) cells, we are now seeing the beginning of pharmacological and therapeutic exploitation. Indeed, the unique properties of this channel family required a long-standing effort for clarifying its structural features and modulatory pathways. It is not surprising that the first (and single for the moment) HCN-related drug in the medical armamentarium was a bradycardic agent, targeting the earliest physiologic function ascribed to $I_{\mathrm{h}}$.

Still, clues emerge for opportunities of future investigation aimed at identifying pharmacological strategies: the prevalence of different $\mathrm{HCN}$ subtypes in organs and tissues, the possibility to target HCN gain or loss of function associated with disease, the feasibility of novel isoform-selective drugs, as well as the discovery of HCN-mediated effects for old medicines. The modulation of neuropathic pain could be likely the next in line precisely because of a combination of these favorable factors. However, the impressive evidence emerging from genetic and molecular studies involving
HCN channels in the CNS could pave the way to unforeseen therapeutic innovation.

\section{Acknowledgments}

We thank Alessandro Mugelli for invaluable support and insightful contribution to authors' research in this field.

\section{Authorship Contributions}

Wrote or contributed to the writing of the manuscript: Sartiani, Masi, Mannaioni, Romanelli, Cerbai.

\section{References}

Abi-Gerges N, Ji GJ, Lu ZJ, Fischmeister R, Hescheler J, and Fleischmann BK (2000) Functional expression and regulation of the hyperpolarization activated nonselective cation current in embryonic stem cell-derived cardiomyocytes. $J$ Physiol 523:377-389.

Abrahamsen B, Zhao J, Asante CO, Cendan CM, Marsh S, Martinez-Barbera JP, Nassar MA, Dickenson AH, and Wood JN (2008) The cell and molecular basis of mechanical, cold, and inflammatory pain. Science 321:702-705.

Accili EA, Proenza C, Baruscotti M, and DiFrancesco D (2002) From funny current to HCN channels: 20 years of excitation. News Physiol Sci 17:32-37.

Acosta C, McMullan S, Djouhri L, Gao L, Watkins R, Berry C, Dempsey K, and Lawson SN (2012) HCN1 and HCN2 in Rat DRG neurons: levels in nociceptors and non-nociceptors, NT3-dependence and influence of CFA-induced skin inflammation on HCN2 and NT3 expression. PLoS One 7:e50442.

Akimoto M, Zhang Z, Boulton S, Selvaratnam R, VanSchouwen B, Gloyd M, Accili EA, Lange OF, and Melacini G (2014) A mechanism for the auto-inhibition of hyperpolarization-activated cyclic nucleotide-gated (HCN) channel opening and its relief by cAMP. J Biol Chem 289:22205-22220.

Alig J, Marger L, Mesirca P, Ehmke H, Mangoni ME, and Isbrandt D (2009) Control of heart rate by cAMP sensitivity of HCN channels. Proc Natl Acad Sci USA 106: 12189-12194.

Alonso A and Llinás RR (1989) Subthreshold $\mathrm{Na}^{+}$-dependent theta-like rhythmicity in stellate cells of entorhinal cortex layer II. Nature 342:175-177.

Alotaibi M, Kahlat K, Nedjadi T, and Djouhri L (2017) Effects of ZD7288, a hyperpolarization-activated cyclic nucleotide-gated (HCN) channel blocker, on term-pregnant rat uterine contractility in vitro. Theriogenology 90:141-146.

Altomare C, Bucchi A, Camatini E, Baruscotti M, Viscomi C, Moroni A and DiFrancesco D (2001) Integrated allosteric model of voltage gating of HCN channels. J Gen Physiol 117:519-532.

Altomare C, Terragni B, Brioschi C, Milanesi R, Pagliuca C, Viscomi C, Moroni A, Baruscotti M, and DiFrancesco D (2003) Heteromeric HCN1-HCN4 channels: comparison with native pacemaker channels from the rabbit sinoatrial node. $J$ Physiol 549:347-359.

Anger KE (2013) Dexmedetomidine: a review of its use for the management of pain agitation, and delirium in the intensive care unit. Curr Pharm Des 19:4003-4013.

Arencibia-Albite F, Vázquez R, Velásquez-Martinez MC, and Jiménez-Rivera CA (2012) Cocaine sensitization inhibits the hyperpolarization-activated cation current Ih and reduces cell size in dopamine neurons of the ventral tegmental area. $J$ Neurophysiol 107:2271-2282.

Arencibia-Albite F, Vázquez-Torres R, and Jiménez-Rivera CA (2017) Cocaine sensitization increases subthreshold activity in dopamine neurons from the ventral tegmental area. J Neurophysiol 117:612-623.

Arinsburg SS, Cohen IS, and Yu HG (2006) Constitutively active Src tyrosine kinase changes gating of HCN4 channels through direct binding to the channel proteins. $J$ Cardiovasc Pharmacol 47:578-586.

Arnsten AF (2011) Prefrontal cortical network connections: key site of vulnerability in stress and schizophrenia. Int $J$ Dev Neurosci 29:215-223.

Arnsten AF and Jin LE (2014) Molecular influences on working memory circuits in dorsolateral prefrontal cortex. Prog Mol Biol Transl Sci 122:211-231.

Bankston JR, Camp SS, DiMaio F, Lewis AS, Chetkovich DM, and Zagotta WN (2012) Structure and stoichiometry of an accessory subunit TRIP8b interaction with hyperpolarization-activated cyclic nucleotide-gated channels. Proc Natl Acad Sci USA 109:7899-7904.

Barbieri M, Varani K, Cerbai E, Guerra L, Li Q, Borea PA, and Mugelli A (1994) Electrophysiological basis for the enhanced cardiac arrhythmogenic effect of isoprenaline in aged spontaneously hypertensive rats. J Mol Cell Cardiol 26:849-860.

Barbuti A, Crespi A, Capilupo D, Mazzocchi N, Baruscotti M, and DiFrancesco D (2009) Molecular composition and functional properties of f-channels in murine embryonic stem cell-derived pacemaker cells. J Mol Cell Cardiol 46:343-351.

Barbuti A, Gravante B, Riolfo M, Milanesi R, Terragni B, and DiFrancesco D (2004) Localization of pacemaker channels in lipid rafts regulates channel kinetics. Circ Res 94:1325-1331.

Barbuti A and Robinson RB (2015) Stem cell-derived nodal-like cardiomyocytes as a novel pharmacologic tool: insights from sinoatrial node development and function. Pharmacol Rev 67:368-388.

Barbuti A, Scavone A, Mazzocchi N, Terragni B, Baruscotti M, and Difrancesco D (2012) A caveolin-binding domain in the HCN4 channels mediates functional interaction with caveolin proteins. J Mol Cell Cardiol 53:187-195.

Barbuti A, Terragni B, Brioschi C, and DiFrancesco D (2007) Localization of f-channels to caveolae mediates specific beta2-adrenergic receptor modulation of rate in sinoatrial myocytes. $\mathrm{J} \mathrm{Mol} \mathrm{Cell} \mathrm{Cardiol} \mathrm{42:71-78.}$

Baruscotti M, Bianco E, Bucchi A, and DiFrancesco D (2016) Current understanding of the pathophysiological mechanisms responsible for inappropriate sinus tachycardia: role of the If "funny" current. J Interv Card Electrophysiol 46:19-28. 
Baruscotti M, Bucchi A, Viscomi C, Mandelli G, Consalez G, Gnecchi-Rusconi T, Montano N, Casali KR, Micheloni S, Barbuti A, et al. (2011) Deep bradycardia and heart block caused by inducible cardiac-specific knockout of the pacemaker channel gene Hcn4. Proc Natl Acad Sci USA 108:1705-1710.

Bédard C, Kröger H, and Destexhe A (2006) Model of low-pass filtering of local field potentials in brain tissue. Phys Rev E Stat Nonlin Soft Matter Phys 73:051911.

Bellingham MC (2013) Pre- and postsynaptic mechanisms underlying inhibition of hypoglossal motor neuron excitability by riluzole. J Neurophysiol 110:1047-1061.

Bender RA, Brewster A, Santoro B, Ludwig A, Hofmann F, Biel M, and Baram TZ (2001) Differential and age-dependent expression of hyperpolarization-activated, cyclic nucleotide-gated cation channel isoforms 1-4 suggests evolving roles in the developing rat hippocampus. Neuroscience 106:689-698.

Bender RA, Kirschstein T, Kretz O, Brewster AL, Richichi C, Rüschenschmidt C, Shigemoto R, Beck H, Frotscher M, and Baram TZ (2007) Localization of HCN1 channels to presynaptic compartments: novel plasticity that may contribute to hippocampal maturation. J Neurosci 27:4697-4706.

Bender RA, Soleymani SV, Brewster AL, Nguyen ST, Beck H, Mathern GW, and Baram TZ (2003) Enhanced expression of a specific hyperpolarizationactivated cyclic nucleotide-gated cation channel (HCN) in surviving dentate gyrus granule cells of human and experimental epileptic hippocampus. $J$ Neurosci 23: $6826-6836$.

Berger T, Senn W, and Lüscher HR (2003) Hyperpolarization-activated current Ih disconnects somatic and dendritic spike initiation zones in layer $\mathrm{V}$ pyramidal neurons. J Neurophysiol 90:2428-2437.

Biel M, Wahl-Schott C, Michalakis S, and Zong X (2009) Hyperpolarization-activated cation channels: from genes to function. Physiol Rev 89:847-885.

Biel S, Aquila M, Hertel B, Berthold A, Neumann T, DiFrancesco D, Moroni A, Thiel G, and Kauferstein S (2016) Mutation in S6 domain of HCN4 channel in patient with suspected Brugada syndrome modifies channel function. Pflugers Arch 468: 1663-1671.

Bischoff U, Bräu ME, Vogel W, Hempelmann G, and Olschewski A (2003) Local anaesthetics block hyperpolarization-activated inward current in rat small dorsal root ganglion neurones. Br J Pharmacol 139:1273-1280.

Blackiston DJ, McLaughlin KA, and Levin M (2009) Bioelectric controls of cell proliferation: ion channels, membrane voltage and the cell cycle. Cell Cycle 8: $3527-3536$

Bogdan R, Goegelein $\mathrm{H}$, and Ruetten $\mathrm{H}$ (2011) Effect of dronedarone on $\mathrm{Na}^{+}, \mathrm{Ca}^{2+}$ and HCN channels. Naunyn Schmiedebergs Arch Pharmacol 383:347-356.

Böhm M, Swedberg K, Komajda M, Borer JS, Ford I, Dubost-Brama A, Lerebours G, and Tavazzi L; SHIFT Investigators (2010) Heart rate as a risk factor in chronic heart failure (SHIFT): the association between heart rate and outcomes in a randomised placebo-controlled trial. Lancet 376:886-894.

Bois P, Bescond J, Renaudon B, and Lenfant J (1996) Mode of action of bradycardic agent, S 16257, on ionic currents of rabbit sinoatrial node cells. Br J Pharmacol 118:1051-1057.

Bois P, Guinamard R, Chemaly AE, Faivre J-F, and Bescond J (2007) Molecular regulation and pharmacology of pacemaker channels. Curr Pharm Des 13: 2338-2349.

Bojak I, Day HC, and Liley DT (2013) Ketamine, propofol, and the EEG: a neural field analysis of HCN1-mediated interactions. Front Comput Neurosci 7:22.

Bolívar JJ, Tapia D, Arenas G, Castañón-Arreola M, Torres H, and Galarraga E (2008) A hyperpolarization-activated, cyclic nucleotide-gated, (Ih-like) cationic current and HCN gene expression in renal inner medullary collecting duct cells. Am J Physiol Cell Physiol 294:C893-C906.

Borel M, Guadagna S, Jang HJ, Kwag J, and Paulsen O (2013) Frequency dependence of CA3 spike phase response arising from h-current properties. Front Cell Neurosci 7:263.

Bosman A, Sartiani L, Spinelli V, Del Lungo M, Stillitano F, Nosi D, Mugelli A, Cerbai E, and Jaconi M (2013) Molecular and functional evidence of HCN4 and caveolin-3 interaction during cardiomyocyte differentiation from human embryonic stem cells. Stem Cells Dev 22:1717-1727.

BoSmith RE, Briggs I, and Sturgess NC (1993) Inhibitory action of ZENECA ZD7288 on whole-cell hyperpolarization activated inward current $\left(\mathrm{I}_{\mathrm{f}}\right)$ in guinea-pig dissociated sinoatrial node cells. Br J Pharmacol 110:313-349.

Boyes J, Bolam JP, Shigemoto R, and Stanford IM (2007) Functional presynaptic HCN channels in the rat globus pallidus. Eur J Neurosci 25:2081-2092.

Branch SY, Chen C, Sharma R, Lechleiter JD, Li S, and Beckstead MJ (2016) Dopaminergic neurons exhibit an age-dependent decline in electrophysiological parameters in the MitoPark mouse model of Parkinson's disease. J Neurosci 36: 4026-4037.

Brandt MC, Endres-Becker J, Zagidullin N, Motloch LJ, Er F, Rottlaender D, Michels G, Herzig S, and Hoppe UC (2009) Effects of KCNE2 on HCN isoforms: distinct modulation of membrane expression and single channel properties. Am J Physiol Heart Circ Physiol 297:H355-H363.

Brewster A, Bender RA, Chen Y, Dube C, Eghbal-Ahmadi M, and Baram TZ (2002) Developmental febrile seizures modulate hippocampal gene expression of hyperpolarization-activated channels in an isoform- and cell-specific manner. J Neurosci 22:4591-4599.

Brewster AL, Chen Y, Bender RA, Yeh A, Shigemoto R, and Baram TZ (2007) Quantitative analysis and subcellular distribution of mRNA and protein expression of the hyperpolarization-activated cyclic nucleotide-gated channels throughout development in rat hippocampus. Cereb Cortex 17:702-712.

Brown HF, DiFrancesco D, and Noble SJ (1979) How does adrenaline accelerate the heart? Nature 280:235-236.

Brummett CM, Hong EK, Janda AM, Amodeo FS, and Lydic R (2011) Perineural dexmedetomidine added to ropivacaine for sciatic nerve block in rats prolongs the duration of analgesia by blocking the hyperpolarization-activated cation current. Anesthesiology 115:836-843.

Bucchi A, Baruscotti M, Nardini M, Barbuti A, Micheloni S, Bolognesi M, and DiFrancesco D (2013) Identification of the molecular site of ivabradine binding to HCN4 channels. PLoS One 8:e53132.
Bucchi A, Tognati A, Milanesi R, Baruscotti M, and DiFrancesco D (2006) Properties of ivabradine-induced block of HCN1 and HCN4 pacemaker channels. J Physiol 572:335-346.

Cacheaux LP, Topf N, Tibbs GR, Schaefer UR, Levi R, Harrison NL, Abbott GW, and Goldstein PA (2005) Impairment of hyperpolarization-activated, cyclic nucleotide-gated channel function by the intravenous general anesthetic propofol. $J$ Pharmacol Exp Ther 315:517-525.

Camici PG, Gloekler S, Levy BI, Skalidis E, Tagliamonte E, Vardas P, and Heusch G (2016) Ivabradine in chronic stable angina: effects by and beyond heart rate reduction. Int $J$ Cardiol 215:1-6.

Caminiti G, Fossati C, Rosano G, and Volterrani M (2016) Addition of ivabradine to betablockers in patients with atrial fibrillation: Effects on heart rate and exercise tolerance. Int $J$ Cardiol 202:73-74.

Cangiano L, Gargini C, Della Santina L, Demontis GC, and Cervetto L (2007) High pass filtering of input signals by the Ih current in a non-spiking neuron, the retinal rod bipolar cell. PLoS One 2:e1327.

Cao JL, Covington IIIHE, Friedman AK, Wilkinson MB, Walsh JJ, Cooper DC, Nestler EJ, and Han MH (2010) Mesolimbic dopamine neurons in the brain reward circuit mediate susceptibility to social defeat and antidepressant action. J Neurosci 30:16453-16458.

Cao XJ and Oertel D (2011) The magnitudes of hyperpolarization-activated and lowvoltage-activated potassium currents co-vary in neurons of the ventral cochlear nucleus. $J$ Neurophysiol 106:630-640

Carlson AE, Rosenbaum JC, Brelidze TI, Klevit RE, and Zagotta WN (2013) Flavonoid regulation of HCN2 channels. J Biol Chem 288:33136-33145.

Carmeliet E (1984) Existence of pacemaker current Ifin human atrial appendage fibres. J Physiol 357:125P

Carmeliet E and Saikawa T (1982) Shortening of the action potential and reduction of pacemaker activity by lidocaine, quinidine, and procainamide in sheep cardiac purkinje fibers: an effect on $\mathrm{Na}$ or K currents? Circ Res 50:257-272.

Carr DB, Andrews GD, Glen WB, and Lavin A (2007) $\alpha 2$-Noradrenergic receptors activation enhances excitability and synaptic integration in rat prefrontal cortex pyramidal neurons via inhibition of HCN currents. $J$ Physiol 584:437-450.

Cavanaugh DJ, Lee H, Lo L, Shields SD, Zylka MJ, Basbaum AI, and Anderson DJ (2009) Distinct subsets of unmyelinated primary sensory fibers mediate behavioral responses to noxious thermal and mechanical stimuli. Proc Natl Acad Sci USA 106: 9075-9080.

Ceconi C, Comini L, Suffredini S, Stillitano F, Bouly M, Cerbai E, Mugelli A and Ferrari $\mathrm{R}$ (2011) Heart rate reduction with ivabradine prevents the global phenotype of left ventricular remodeling. Am J Physiol Heart Circ Physiol 300: H366-H373.

Cerbai E, Barbieri M, and Mugelli A (1994) Characterization of the hyperpolarization-activated current, I(f), in ventricular myocytes isolated from hypertensive rats. J Physiol 481:585-591.

Cerbai E, Crucitti A, Sartiani L, De Paoli P, Pino R, Rodriguez ML, Gensini G, and Mugelli A (2000) Long-term treatment of spontaneously hypertensive rats with losartan and electrophysiological remodeling of cardiac myocytes. Cardiovasc Res 45:388-396.

Cerbai E, De Paoli P, Sartiani L, Lonardo G, and Mugelli A (2003) Treatment with irbesartan counteracts the functional remodeling of ventricular myocytes from hypertensive rats. $J$ Cardiovasc Pharmacol 41:804-812.

Cerbai E and Mugelli A (2006) I(f) in non-pacemaker cells: role and pharmacological implications. Pharmacol Res 53:416-423.

Cerbai E, Pino R, Porciatti F, Sani G, Toscano M, Maccherini M, Giunti G, and Mugelli A (1997) Characterization of the hyperpolarization-activated current, I (f), in ventricular myocytes from human failing heart. Circulation 95:568-571.

Cerbai E, Pino R, Rodriguez ML, and Mugelli A (1999a) Modulation of the pacemaker current If by beta-adrenoceptor subtypes in ventricular myocytes isolated from hypertensive and normotensive rats. Cardiovasc Res 42:121-129.

Cerbai E, Pino R, Sartiani L, and Mugelli A (1999b) Influence of postnataldevelopment on $\mathrm{I}(\mathrm{f})$ occurrence and properties in neonatal rat ventricular myocytes. Cardiovasc Res 42:416-423.

Cerbai E, Sartiani L, DePaoli P, Pino R, Maccherini M, Bizzarri F, DiCiolla F, Davol G, Sani G, and Mugelli A (2001) The properties of the pacemaker current I(F)in human ventricular myocytes are modulated by cardiac disease. $\mathrm{J} \mathrm{Mol} \mathrm{Cell} \mathrm{Cardiol}$ 33:441-448.

Cervetto L, Demontis GC, and Gargini C (2007) Cellular mechanisms underlying the pharmacological induction of phosphenes. Br J Pharmacol 150:383-390.

Chan Y-C, Wang K, Au KW, Lau C-P, Tse H-F, and Li RA (2009) Probing the bradycardic drug binding receptor of $\mathrm{HCN}$-encoded pacemaker channels [published correction appears in Pflugers Arch (2010) 459:519]. Pflugers Arch 459:25-38.

Chandler NJ, Greener ID, Tellez JO, Inada S, Musa H, Molenaar P, Difrancesco D, Baruscotti M, Longhi R, Anderson RH, et al. (2009) Molecular architecture of the human sinus node: insights into the function of the cardiac pacemaker. Circulation 119:1562-1575.

Chaplan SR, Guo HQ, Lee DH, Luo L, Liu C, Kuei C, Velumian AA, Butler MP, Brown SM, and Dubin AE (2003) Neuronal hyperpolarization-activated pacemaker channels drive neuropathic pain. $J$ Neurosci 23:1169-1178.

Chen C (1997) Hyperpolarization-activated current (Ih) in primary auditory neurons. Hear Res 110:179-190.

Chen K, Aradi I, Thon N, Eghbal-Ahmadi M, Baram TZ, and Soltesz I (2001) Persistently modified h-channels after complex febrile seizures convert the seizure-induced enhancement of inhibition to hyperexcitability. Nat Med 7:331-337.

Chen S, Liang MC, Chia JN, Ngsee JK, and Ting AE (2001a) Rab8b and its interacting partner TRIP8b are involved in regulated secretion in AtT20 cells. J Biol Chem 276:13209-13216.

Chen S, Wang J, and Siegelbaum SA (2001b) Properties of hyperpolarizationactivated pacemaker current defined by coassembly of HCN1 and HCN2 subunits and basal modulation by cyclic nucleotide. J Gen Physiol 117:491-504.

Chen X, Shu S, and Bayliss DA (2009a) HCN1 channel subunits are a molecular substrate for hypnotic actions of ketamine. J Neurosci 29:600-609. 
Chen X, Shu S, Kennedy DP, Willcox SC, and Bayliss DA (2009b) Subunit-specific effects of isoflurane on neuronal Ih in HCN1 knockout mice. $J$ Neurophysiol 101: $129-140$.

Chen X, Sirois JE, Lei Q, Talley EM, Lynch IIIC, and Bayliss DA (2005) HCN subunit-specific and cAMP-modulated effects of anesthetics on neuronal pacemaker currents. J Neurosci 25:5803-5814.

Cheng L, Kinard K, Rajamani R, and Sanguinetti MC (2007) Molecular mapping of the binding site for a blocker of hyperpolarization-activated, cyclic nucleotidemodulated pacemaker channels. J Pharmacol Exp Ther 322:931-939.

Cheng Q and Zhou Y (2013) Novel role of KT5720 on regulating hyperpolarizationactivated cyclic nucleotide-gated channel activity and dorsal root ganglion neuron excitability. DNA Cell Biol 32:320-328.

Chow SS, Van Petegem F, and Accili EA (2012) Energetics of cyclic AMP binding to HCN channel C terminus reveal negative cooperativity. J Biol Chem 287:600-606.

Christoffels VM, Smits GJ, Kispert A, and Moorman AF (2010) Development of the pacemaker tissues of the heart. Circ Res 106:240-254.

Craven KB, Olivier NB, and Zagotta WN (2008) C-terminal movement during gating in cyclic nucleotide-modulated channels. J Biol Chem 283:14728-14738.

Craven KB and Zagotta WN (2006) CNG and HCN channels: two peas, one pod. Annu Rev Physiol 68:375-401.

Custodis F, Baumhäkel M, Schlimmer N, List F, Gensch C, Böhm M, and Laufs U (2008) Heart rate reduction by ivabradine reduces oxidative stress, improves endothelial function, and prevents atherosclerosis in apolipoprotein E-deficient mice. Circulation 117:2377-2387.

Dalle C and Eisenach JC (2005) Peripheral block of the hyperpolarization-activated cation current (Ih) reduces mechanical allodynia in animal models of postoperative and neuropathic pain. Reg Anesth Pain Med 30:243-248.

Decher N, Bundis F, Vajna R, and Steinmeyer K (2003) KCNE2 modulates current amplitudes and activation kinetics of HCN4: influence of KCNE family members on HCN4 currents. Pflugers Arch 446:633-640.

Dedkov EI, Zheng W, Christensen LP, Weiss RM, Mahlberg-Gaudin F, and Tomanek RJ (2007) Preservation of coronary reserve by ivabradine-induced reduction in heart rate in infarcted rats is associated with decrease in perivascular collagen. $A m$ J Physiol Heart Circ Physiol 293:H590-H598.

Della Santina L, Bouly M, Asta A, Demontis GC, Cervetto L, and Gargini C (2010) Effect of HCN channel inhibition on retinal morphology and function in normal and dystrophic rodents. Invest Ophthalmol Vis Sci 51:1016-1023.

Del Lungo M, Melchiorre M, Guandalini L, Sartiani L, Mugelli A, Koncz I, Szel T, Varro A, Romanelli MN, and Cerbai E (2012) Novel blockers of hyperpolarizationactivated current with isoform selectivity in recombinant cells and native tissue. $\mathrm{Br}$ $J$ Pharmacol 166:602-616.

Delpón E, Valenzuela C, Pérez O, Franqueza L, Gay P, Snyders DJ, and Tamargo J (1996) Mechanisms of block of a human cloned potassium channel by the enantiomers of a new bradycardic agent: S-16257-2 and S-16260-2. Br J Pharmacol 117:1293-1301.

De Mello WC (2004) Heart failure: how important is cellular sequestration? The role of the renin-angiotensin-aldosterone system. $J$ Mol Cell Cardiol 37:431-438.

Deng T, Zhang Q, Wang Q, Zhong X, and Li L (2015) Changes in hyperpolarizationactivated cyclic nucleotide-gated channel expression and activity in bladder interstitial cells of Cajal from rats with detrusor overactivity. Int Urogynecol J Pelvic Floor Dysfunct 26:1139-1145.

Descoeur J, Pereira V, Pizzoccaro A, Francois A, Ling B, Maffre V, Couette B, Busserolles J, Courteix C, Noel J, et al. (2011) Oxaliplatin-induced cold hypersensitivity is due to remodelling of ion channel expression in nociceptors. EMBO Mol Med $\mathbf{3}$ : $266-278$

Dias da Silva VJ, Tobaldini E, Rocchetti M, Wu MA, Malfatto G, Montano N, and Zaza A (2015) Modulation of sympathetic activity and heart rate variability by ivabradine. Cardiovasc Res 108:31-38.

Dibbens LM, Reid CA, Hodgson B, Thomas EA, Phillips AM, Gazina E, Cromer BA Clarke AL, Baram TZ, Scheffer IE, et al. (2010) Augmented currents of an HCN2 variant in patients with febrile seizure syndromes. Ann Neurol 67:542-546.

DiFrancesco D (1981a) A new interpretation of the pace-maker current in calf Purkinje fibres. J Physiol 314:359-376.

DiFrancesco D (1981b) A study of the ionic nature of the pace-maker current in calf Purkinje fibres. J Physiol 314:377-393.

DiFrancesco D (1982) Block and activation of the pace-maker channel in calf purkinje fibres: effects of potassium, caesium and rubidium. J Physiol 329:485-507.

DiFrancesco D (1986) Characterization of single pacemaker channels in cardiac sinoatrial node cells. Nature 324:470-473.

DiFrancesco D (1993) Pacemaker mechanisms in cardiac tissue. Annu Rev Physiol 55:455-472.

DiFrancesco D (1994) Some properties of the UL-FS 49 block of the hyperpolarization-activated current (i(f)) in sino-atrial node myocytes. Pflugers Arch 427:64-70.

DiFrancesco D (1999) Dual allosteric modulation of pacemaker (f) channels by cAMP and voltage in rabbit SA node. J Physiol 515:367-376.

DiFrancesco D (2006) Serious workings of the funny current. Prog Biophys Mol Biol 90:13-25.

DiFrancesco D (2010) The role of the funny current in pacemaker activity. Circ Res 106: $434-446$

DiFrancesco D and Borer JS (2007) The funny current: cellular basis for the control of heart rate. Drugs 67 (Suppl 2):15-24.

DiFrancesco D, Ducouret P, and Robinson RB (1989) Muscarinic modulation of cardiac rate at low acetylcholine concentrations. Science 243:669-671.

DiFrancesco D, Ferroni A, Mazzanti M, and Tromba C (1986) Properties of the hyperpolarizing-activated current (if) in cells isolated from the rabbit sino-atrial node. J Physiol 377:61-88.

DiFrancesco D and Noble D (2012) The funny current has a major pacemaking role in the sinus node. Heart Rhythm 9:299-301.

DiFrancesco D and Tortora P (1991) Direct activation of cardiac pacemaker channels by intracellular cyclic AMP. Nature 351:145-147.
DiFrancesco JC, Barbuti A, Milanesi R, Coco S, Bucchi A, Bottelli G, Ferrarese C, Franceschetti S, Terragni B, Baruscotti M, et al. (2011) Recessive loss-of-function mutation in the pacemaker HCN2 channel causing increased neuronal excitability in a patient with idiopathic generalized epilepsy. J Neurosci 31:17327-17337.

DiFrancesco JC and DiFrancesco D (2015) Dysfunctional HCN ion channels in neurological diseases. Front Cell Neurosci 6:174.

Dobrzynski H, Anderson RH, Atkinson A, Borbas Z, D'Souza A, Fraser JF, Inada S, Logantha SJ, Monfredi O, Morris GM, et al. (2013) Structure, function and clinical relevance of the cardiac conduction system, including the atrioventricular ring and outflow tract tissues. Pharmacol Ther 139:260-288.

D'Souza A, Bucchi A, Johnsen AB, Logantha SJ, Monfredi O, Yanni J, Prehar S, Hart G, Cartwright E, Wisloff U, et al. (2014) Exercise training reduces resting heart rate via downregulation of the funny channel HCN4. Nat Commun 5:3775.

D'Souza A, Sharma S, and Boyett MR (2015) CrossTalk opposing view: bradycardia in the trained athlete is attributable to a downregulation of a pacemaker channel in the sinus node. $J$ Physiol 593:1749-1751.

Dufour MA, Woodhouse A, and Goaillard JM (2014) Somatodendritic ion channel expression in substantia nigra pars compacta dopaminergic neurons across postnatal development. J Neurosci Res 92:981-999.

Dunlop J, Vasilyev D, Lu P, Cummons T, and Bowlby MR (2009) Hyperpolarizationactivated cyclic nucleotide-gated (HCN) channels and pain. Curr Pharm Des 15: 1767-1772.

Dyhrfjeld-Johnsen J, Morgan RJ, and Soltesz I (2009) Double Trouble? Potential for hyperexcitability following both channelopathic up- and downregulation of $\mathrm{I}(\mathrm{h})$ in epilepsy. Front Neurosci 3:25-33.

El-Kholy W, MacDonald PE, Fox JM, Bhattacharjee A, Xue T, Gao X, Zhang Y, Stieber J, Li RA, Tsushima RG, et al. (2007) Hyperpolarization-activated cyclic nucleotide-gated channels in pancreatic beta-cells. Mol Endocrinol 21:753-764.

Emery EC, Young GT, Berrocoso EM, Chen L, and McNaughton PA (2011) HCN2 ion channels play a central role in inflammatory and neuropathic pain. Science $\mathbf{3 3 3}$ : 1462-1466.

Emery EC, Young GT, and McNaughton PA (2012) HCN2 ion channels: an emerging role as the pacemakers of pain. Trends Pharmacol Sci 33:456-463.

Engel D and Seutin V (2015) High dendritic expression of Ih in the proximity of the axon origin controls the integrative properties of nigral dopamine neurons. $J$ Physiol 593:4905-4922.

Fabbri A, Fantini M, Wilders R, and Severi S (2017) Computational analysis of the human sinus node action potential: model development and effects of mutations. $J$ Physiol 595:2365-2396.

Fan X, Chen Y, Wu P, Xing J, Chen H, Song T, Yang J, Zhang J, and Huang C (2011) Novel electropharmacological activity of amiodarone on human HCN channels heterologously expressed in the Xenopus oocytes. Eur J Pharmacol 669:15-23.

Fenske S, Mader R, Scharr A, Paparizos C, Cao-Ehlker X, Michalakis S, Shaltiel L, Weidinger M, Stieber J, Feil S, et al. (2011) HCN3 contributes to the ventricular action potential waveform in the murine heart. Circ Res 109:1015-1023.

Fernández-Velasco M, Goren N, Benito G, Blanco-Rivero J, Boscá L, and Delgado C (2003) Regional distribution of hyperpolarization-activated current (If) and hyperpolarization-activated cyclic nucleotide-gated channel mRNA expression in ventricular cells from control and hypertrophied rat hearts. J Physiol 553:395-405.

Flynn GE, Black KD, Islas LD, Sankaran B, and Zagotta WN (2007) Structure and rearrangements in the carboxy-terminal region of $\mathrm{SpIH}$ channels. Structure $\mathbf{1 5}$ 671-682.

Flynn GE and Zagotta WN (2011) Molecular mechanism underlying phosphatidylinositol 4,5-bisphosphate-induced inhibition of SpIH channels. J Biol Chem 286: 15535-15542.

Fogle KJ, Lyashchenko AK, Turbendian HK, and Tibbs GR (2007) HCN pacemaker channel activation is controlled by acidic lipids downstream of diacylglycerol kinase and phospholipase A2. J Neurosci 27:2802-2814

Fox K, Ford I, Steg PG, Tardif JC, Tendera M, and Ferrari R; SIGNIFY Investigators (2014) Ivabradine in stable coronary artery disease without clinical heart failure. $N$ Engl J Med 371:1091-1099.

Fox K, Ford I, Steg PG, Tardif JC, Tendera M, and Ferrari R; SIGNIFY Investigators (2015) Bradycardia and atrial fibrillation in patients with stable coronary artery disease treated with ivabradine: an analysis from the SIGNIFY study. Eur Heart $J$ 36:3291-3296.

Fox K, Ford I, Steg PG, Tendera M, Robertson M, and Ferrari R; BEAUTIFUL investigators (2008) Heart rate as a prognostic risk factor in patients with coronary artery disease and left-ventricular systolic dysfunction (BEAUTIFUL): a subgroup analysis of a randomised controlled trial. Lancet 372:817-821.

Franz O, Liss B, Neu A, and Roeper J (2000) Single-cell mRNA expression of HCN1 correlates with a fast gating phenotype of hyperpolarization-activated cyclic nucleotide-gated ion channels (Ih) in central neurons. Eur J Neurosci 12:2685-2693.

Friedman AK, Walsh JJ, Juarez B, Ku SM, Chaudhury D, Wang J, Li X, Dietz DM, Pan N, Vialou VF, et al. (2014) Enhancing depression mechanisms in midbrain dopamine neurons achieves homeostatic resilience. Science 344:313-319.

Funahashi M, Higuchi H, Miyawaki T, Shimada M, and Matsuo R (2001) Propofol suppresses a hyperpolarization-activated inward current in rat hippocampal CA1 neurons. Neurosci Lett 311:177-180.

Funahashi M, Mitoh Y, and Matsuo R (2004) The sensitivity of hyperpolarizationactivated cation current (Ih) to propofol in rat area postrema neurons. Brain Res 1015:198-201.

Fyk-Kolodziej B and Pourcho RG (2007) Differential distribution of hyperpolarizationactivated and cyclic nucleotide-gated channels in cone bipolar cells of the rat retina. J Comp Neurol 501:891-903.

Galligan JJ, Tatsumi H, Shen KZ, Surprenant A, and North RA (1990) Cation current activated by hyperpolarization (IH) in guinea pig enteric neurons. Am J Physiol 259:G966-G972.

Gamo NJ, Lur G, Higley MJ, Wang M, Paspalas CD, Vijayraghavan S, Yang Y, Ramos BP, Peng K, Kata A, et al. (2015) Stress impairs prefrontal cortical function via D1 dopamine receptor interactions with hyperpolarization-activated cyclic nucleotide-gated channels. Biol Psychiatry 78:860-870. 
Gao LL, McMullan S, Djouhri L, Acosta C, Harper AA, and Lawson SN (2012) Expression and properties of hyperpolarization-activated current in rat dorsal root ganglion neurons with known sensory function. J Physiol 590:4691-4705.

Gasparini S and DiFrancesco D (1997) Action of the hyperpolarization-activated current (Ih) blocker ZD 7288 in hippocampal CA1 neurons. Pflugers Arch 435 99-106.

Gauss R, Seifert R, and Kaupp UB (1998) Molecular identification of a hyperpolarization-activated channel in sea urchin sperm. Nature 393:583-587.

Giocomo LM and Hasselmo ME (2009) Knock-out of HCN1 subunit flattens dorsalventral frequency gradient of medial entorhinal neurons in adult mice. $J$ Neurosci 29:7625-7630.

Giocomo LM, Hussaini SA, Zheng F, Kandel ER, Moser MB, and Moser EI (2011) Grid cells use HCN1 channels for spatial scaling. Cell 147:1159-1170.

Goertz RB, Wanat MJ, Gomez JA, Brown ZJ, Phillips PE, and Paladini CA (2015) Cocaine increases dopaminergic neuron and motor activity via midbrain $\alpha 1$ adrenergic signaling. Neuropsychopharmacology 40:1151-1162.

Goethals M, Raes A, and van Bogaert PP (1993) Use-dependent block of the pacemaker current I(f) in rabbit sinoatrial node cells by zatebradine (UL-FS 49). On the mode of action of sinus node inhibitors. Circulation 88:2389-2401.

González B, Rivero-Echeto C, Muñiz JA, Cadet JL, García-Rill E, Urbano FJ, and Bisagno V (2016) Methamphetamine blunts $\mathrm{Ca}(2+)$ currents and excitatory synaptic transmission through D1/5 receptor-mediated mechanisms in the mouse medial prefrontal cortex. Addict Biol 21:589-602.

Good CH, Hoffman AF, Hoffer BJ, Chefer VI, Shippenberg TS, Bäckman CM, Larsson NG, Olson L, Gellhaar S, Galter D, et al (2011) Impaired nigrostriatal function precedes behavioral deficits in a genetic mitochondrial model of Parkinson's disease. FASEB $J$ 25:1333-1344

Gravante B, Barbuti A, Milanesi R, Zappi I, Viscomi C, and DiFrancesco D (2004) Interaction of the pacemaker channel HCN1 with filamin A. J Biol Chem 279: 43847-43853.

Greenwood IA and Prestwich SA (2002) Characteristics of hyperpolarizationactivated cation currents in portal vein smooth muscle cells. Am J Physiol Cell Physiol 282:C744-C753.

Halliwell JV and Adams PR (1982) Voltage-clamp analysis of muscarinic excitation in hippocampal neurons. Brain Res 250:71-92.

Hancox JC, Melgari D, Dempsey CE, Brack KE, Mitcheson J, and Ng GA (2015) hERG potassium channel inhibition by ivabradine may contribute to QT prolongation and risk of torsades de pointes. Ther Adv Drug Saf 6:177-179.

Harnett MT, Magee JC, and Williams SR (2015) Distribution and function of HCN channels in the apical dendritic tuft of neocortical pyramidal neurons. $J$ Neurosci 35:1024-1037.

Hashitani H, Nguyen MJ, Noda H, Mitsui R, Higashi R, Ohta K, Nakamura KI, and Lang RJ (2017) Interstitial cell modulation of pyeloureteric peristalsis in the mouse renal pelvis examined using FIBSEM tomography and calcium indicators. Pflugers Arch 469:797-813.

Hawkins VE, Hawryluk JM, Takakura AC, Tzingounis AV, Moreira TS, and Mulkey DK (2015) HCN channels contribute to serotonergic modulation of ventral surface chemosensitive neurons and respiratory activity. $J$ Neurophysiol 113:1195-1205.

He C, Chen F, Li B, and Hu Z (2014) Neurophysiology of HCN channels: from cellular functions to multiple regulations. Prog Neurobiol 112:1-23.

He JQ, Ma Y, Lee Y, Thomson JA, and Kamp TJ (2003) Human embryonic stem cells develop into multiple types of cardiac myocytes: action potential characterization. Circ Res 93:32-39.

He P, Deng J, Zhong X, Zhou Z, Song B, and Li L (2012) Identification of a hyperpolarization-activated cyclic nucleotide-gated channel and its subtypes in the urinary bladder of the rat. Urology 79:1411.e7-e13.

Herrmann S, Hofmann F, Stieber J, and Ludwig A (2012) HCN channels in the heart: lessons from mouse mutants. Br J Pharmacol 166:501-509.

Herrmann S, Schnorr S, and Ludwig A (2015) HCN channels-modulators of cardiac and neuronal excitability. Int J Mol Sci 16:1429-1447.

Herrmann S, Stieber J, Stöckl G, Hofmann F, and Ludwig A (2007) HCN4 provides a 'depolarization reserve' and is not required for heart rate acceleration in mice. EMBO J 26:4423-4432.

Heubach JF, Graf EM, Leutheuser J, Bock M, Balana B, Zahanich I, Christ T, Boxberger S, Wettwer E, and Ravens U (2004) Electrophysiological properties of human mesenchymal stem cells. $J$ Physiol 554:659-672.

Heuermann RJ, Jaramillo TC, Ying SW, Suter BA, Lyman KA, Han Y, Lewis AS, Hampton TG, Shepherd GM, Goldstein PA, et al. (2016) Reduction of thalamic and cortical Ih by deletion of TRIP8b produces a mouse model of human absence epilepsy. Neurobiol Dis 85:81-92.

Higuchi H, Funahashi M, Miyawaki T, Mitoh Y, Kohjitani A, Shimada M, and Matsuo R (2003) Suppression of the hyperpolarization-activated inward current contributes to the inhibitory actions of propofol on rat CA1 and CA3 pyramidal neurons. Neurosci Res 45:459-472.

Ho WK, Brown HF, and Noble D (1994) High selectivity of the i(f) channel to Na+ and $\mathrm{K}+$ in rabbit isolated sinoatrial node cells. Pflugers Arch 426:68-74

Hofmann F, Fabritz L, Stieber J, Schmitt J, Kirchhof P, Ludwig A, and Herrmann S (2012) Ventricular HCN channels decrease the repolarization reserve in the hypertrophic heart. Cardiovasc Res 95:317-326.

Hoppe UC and Beuckelmann DJ (1998) Modulation of the hyperpolarizationactivated inward current (If) by antiarrhythmic agents in isolated human atrial myocytes. Naunyn Schmiedebergs Arch Pharmacol 358:635-640.

Hoshi N, Takahashi H, Shahidullah M, Yokoyama S, and Higashida H (1998) KCR1, a membrane protein that facilitates functional expression of non-inactivating $\mathrm{K}^{+}$ currents associates with rat EAG voltage-dependent $\mathrm{K}^{+}$channels. J Biol Chem 273 : 23080-23085.

Hu T, Liu N, Lv M, Ma L, Peng H, Peng S, and Liu T (2016) Lidocaine inhibits HCN currents in rat spinal substantia gelatinosa neurons. Anesth Analg 122:1048-1059.

Huang J, Huang A, Zhang Q, Lin YC, and Yu HG (2008) Novel mechanism for suppression of hyperpolarization-activated cyclic nucleotide-gated pacemaker channels by receptor-like tyrosine phosphatase-alpha. J Biol Chem 283:29912-29919.
Huang X, Yang P, Yang Z, Zhang H, and Ma A (2016) Age-associated expression of HCN channel isoforms in rat sinoatrial node. Exp Biol Med (Maywood) 241 331-339.

Huang Z, Li G, Aguado C, Lujan R, and Shah MM (2017) HCN1 channels reduce the rate of exocytosis from a subset of cortical synaptic terminals. Sci Rep 7:40257.

Huang Z, Lujan R, Kadurin I, Uebele VN, Renger JJ, Dolphin AC, and Shah MM (2011) Presynaptic HCN1 channels regulate Cav3.2 activity and neurotransmission at select cortical synapses. Nat Neurosci 14:478-486.

Huang Z, Walker MC, and Shah MM (2009) Loss of dendritic HCN1 subunits enhances cortical excitability and epileptogenesis. J Neurosci 29:10979-10988.

Hurtado R, Bub G, and Herzlinger D (2014) A molecular signature of tissues with pacemaker activity in the heart and upper urinary tract involves coexpressed hyperpolarization-activated cation and T-type Ca2+ channels. FASEB $J$ 28: $730-739$.

Hutcheon B, Miura RM, and Puil E (1996) Models of subthreshold membrane resonance in neocortical neurons. $J$ Neurophysiol 76:698-714.

Inyushin MU, Arencibia-Albite F, Vázquez-Torres R, Vélez-Hernández ME and Jiménez-Rivera CA (2010) Alpha-2 noradrenergic receptor activation inhibits the hyperpolarization-activated cation current (Ih) in neurons of the ventral tegmental area. Neuroscience 167:287-297.

Isbister GK, Heppell SP, Page CB, and Ryan NM (2017) Adult clonidine overdose: prolonged bradycardia and central nervous system depression, but not severe toxicity. Clin Toxicol (Phila) 55:187-192.

Jackson HA, Marshall CR, and Accili EA (2007) Evolution and structural diversification of hyperpolarization-activated cyclic nucleotide-gated channel genes. Physiol Genomics 29:231-245.

Jackson IILR, Rathakrishnan B, Campbell K, Thomas KL, Piccini JP, Bahnson T, Stiber JA, and Daubert JP (2017) Sinus node dysfunction and atrial fibrillation: a reversible phenomenon? Pacing Clin Electrophysiol 40:442-450.

Jafri MS and Weinreich D (1998) Substance P regulates Ih via a NK-1 receptor in vagal sensory neurons of the ferret. $J$ Neurophysiol 79:769-777.

Janigro D, Martenson ME, and Baumann TK (1997) Preferential inhibition of Th in rat trigeminal ganglion neurons by an organic blocker. J Membr Biol 160:101-109.

Jiang YQ, Xing GG, Wang SL, Tu HY, Chi YN, Li J, Liu FY, Han JS, and Wan Y (2008) Axonal accumulation of hyperpolarization-activated cyclic nucleotide-gated cation channels contributes to mechanical allodynia after peripheral nerve injury in rat. Pain 137:495-506.

Jonas JC, Plant TD, and Henquin JC (1992) Imidazoline antagonists of alpha 2 -adrenoceptors increase insulin release in vitro by inhibiting ATP-sensitive $\mathrm{K}^{+}$ channels in pancreatic beta-cells. Br J Pharmacol 107:8-14.

Kannel WB, Kannel C, Paffenbarger Jr.RS, and Cupples LA (1987) Heart rate and cardiovascular mortality: the Framingham Study. Am Heart J 113:1489-1494.

Kanyshkova T, Pawlowski M, Meuth P, Dubé C, Bender RA, Brewster AL, Baumann A, Baram TZ, Pape HC, and Budde T (2009) Postnatal expression pattern of HCN channel isoforms in thalamic neurons: relationship to maturation of thalamocortica oscillations. J Neurosci 29:8847-8857.

Kase D and Imoto K (2012) The role of HCN channels on membrane excitability in the nervous system. J Signal Transduct 2012:619747.

Kaupp UB and Seifert R (2001) Molecular diversity of pacemaker ion channels. Annu Rev Physiol 63:235-257.

Kaupp UB and Seifert R (2002) Cyclic nucleotide-gated ion channels. Physiol Rev 82 769-824.

Khurana S, Liu Z, Lewis AS, Rosa K, Chetkovich D, and Golding NL (2012) An essential role for modulation of hyperpolarization-activated current in the development of binaural temporal precision. J Neurosci 32:2814-2823.

Kim CS, Chang PY, and Johnston D (2012) Enhancement of dorsal hippocampal activity by knockdown of HCN1 channels leads to anxiolytic- and antidepressantlike behaviors. Neuron 75:503-516.

Kim TH, Kim HI, Kim J, Park M, and Song J-H (2011) Effects of minocycline on Na+ currents in rat dorsal root ganglion neurons. Brain Res 1370:34-42.

Kim YH and Holt JR (2013) Functional contributions of HCN channels in the primary auditory neurons of the mouse inner ear. J Gen Physiol 142:207-223.

Kimura K, Kitano J, Nakajima Y, and Nakanishi S (2004) Hyperpolarizationactivated, cyclic nucleotide-gated HCN2 cation channel forms a protein assembly with multiple neuronal scaffold proteins in distinct modes of protein-protein interaction. Genes Cells 9:631-640.

Knaus A, Zong X, Beetz N, Jahns R, Lohse MJ, Biel M, and Hein L (2007) Direct inhibition of cardiac hyperpolarization-activated cyclic nucleotide-gated pacemaker channels by clonidine. Circulation 115:872-880.

Knoll AT, Halladay LR, Holmes AJ, and Levitt P (2016) Quantitative trait loci and a novel genetic candidate for fear learning. J Neurosci 36:6258-6268.

Ko KW, Rasband MN, Meseguer V, Kramer RH, and Golding NL (2016) Serotonin modulates spike probability in the axon initial segment through HCN channels. Nat Neurosci 19:826-834.

Kobinger W (1987) History of 'specific bradycardic agents'. Eur Heart $J \mathbf{8}$ (Suppl L): $5-6$.

Kobinger W and Lillie C (1987) Specific bradycardic agents-a novel pharmacological class? Eur Heart $J \mathbf{8}$ (Suppl L):7-15.

Kojima A, Ito Y, Kitagawa H, and Matsuura H (2015) Ionic mechanisms underlying the negative chronotropic action of propofol on sinoatrial node automaticity in guinea pig heart. Br J Pharmacol 172:799-814.

Koncz I, Szél T, Bitay M, Cerbai E, Jaeger K, Fülöp F, Jost N, Virág L, Orvos P, Tálosi L, et al. (2011) Electrophysiological effects of ivabradine in dog and human cardiac preparations: potential antiarrhythmic actions. Eur J Pharmacol 668 $419-426$

Kosiuk J, Lindemann F, Hindricks G, and Bollmann A (2016) Need for further studies on ivabradine in patients with persistent atrial fibrillation. Int $J$ Cardio 223:915-916.

Kotani Y, Shimazawa M, Yoshimura S, Iwama T, and Hara H (2008) The experimental and clinical pharmacology of propofol, an anesthetic agent with neuroprotective properties. CNS Neurosci Ther 14:95-106. 
Kuner R (2010) Central mechanisms of pathological pain. Nat Med 16:1258-1266. Kuwabara Y, Kuwahara K, Takano M, Kinoshita H, Arai Y, Yasuno S, Nakagawa Y, Igata S, Usami S, Minami T, et al. (2013) Increased expression of HCN channels in the ventricular myocardium contributes to enhanced arrhythmicity in mouse failing hearts. J Am Heart Assoc 2:e000150.

Lai LP, Su MJ, Lin JL, Tsai CH, Lin FY, Chen YS, Hwang JJ, Huang SK, Tseng YZ, and Lien WP (1999) Measurement of funny current (I(f)) channel mRNA in human atrial tissue: correlation with left atrial filling pressure and atrial fibrillation. $J$ Cardiovasc Electrophysiol 10:947-953.

Lau YT, Wong CK, Luo J, Leung LH, Tsang PF, Bian ZX, and Tsang SY (2011) Effects of hyperpolarization-activated cyclic nucleotide-gated (HCN) channel blockers on the proliferation and cell cycle progression of embryonic stem cells. Pflugers Arch 461:191-202.

Leao RN, Sun H, Svahn K, Berntson A, Youssoufian M, Paolini AG, Fyffe RE and Walmsley B (2006) Topographic organization in the auditory brainstem of juvenile mice is disrupted in congenital deafness. $J$ Physiol 571:563-578.

Lee CH and MacKinnon R (2017) Structures of the human HCN1 hyperpolarizationactivated channel. Cell 168:111-120.e11.

Lees-Miller JP, Guo J, Wang Y, Perissinotti LL, Noskov SY, and Duff HJ (2015) Ivabradine prolongs phase 3 of cardiac repolarization and blocks the hERG1 (KCNH2) current over a concentration-range overlapping with that required to block HCN4. J Mol Cell Cardiol 85:71-78.

Leist M, Datunashvilli M, Kanyshkova T, Zobeiri M, Aissaoui A, Cerina M, Romanell MN, Pape HC, and Budde T (2016) Two types of interneurons in the mouse lateral geniculate nucleus are characterized by different h-current density. Sci Rep 6 24904.

Levin M (2014) Molecular bioelectricity: how endogenous voltage potentials control cell behavior and instruct pattern regulation in vivo. Mol Biol Cell 25 3835-3850.

Lewis AS, Schwartz E, Chan CS, Noam Y, Shin M, Wadman WJ, Surmeier DJ, Baram TZ, Macdonald RL, and Chetkovich DM (2009) Alternatively spliced isoforms of TRIP8b differentially control h channel trafficking and function. $J \mathrm{Neu}$ rosci 29:6250-6265.

Lewis AS, Vaidya SP, Blaiss CA, Liu Z, Stoub TR, Brager DH, Chen X, Bender RA, Estep CM, Popov AB, et al. (2011) Deletion of the hyperpolarization-activated cyclic nucleotide-gated channel auxiliary subunit TRIP8b impairs hippocampal Ih localization and function and promotes antidepressant behavior in mice. $J \mathrm{Neu}$ rosci 31:7424-7440.

Lezoualc'h F, Steplewski K, Sartiani L, Mugelli A, Fischmeister R, and Bril A (2007) Quantitative mRNA analysis of serotonin 5-HT4 receptor isoforms, calcium handling proteins and ion channels in human atrial fibrillation. Biochem Biophys Res Commun 357:218-224.

Li CH, Zhang Q, Teng B, Mustafa SJ, Huang JY, and Yu HG (2008a) Src tyrosine kinase alters gating of hyperpolarization-activated HCN4 pacemaker channel through Tyr531. Am J Physiol Cell Physiol 294:C355-C362.

Li GR and Deng XL (2011) Functional ion channels in stem cells. World J Stem Cells 3:19-24.

Li H, Zhou Y, Jiang B, Zhao X, Li X, Yang X, and Jiang W (2014a) Dual effects of amiodarone on pacemaker currents in hypertrophied ventricular myocytes isolated from spontaneously hypertensive rats. Clin Exp Pharmacol Physiol 41:698-707.

Li N, Csepe TA, Hansen BJ, Dobrzynski H, Higgins RS, Kilic A, Mohler PJ, Janssen PM, Rosen MR, Biesiadecki BJ, et al. (2015a) Molecular mapping of sinoatrial node HCN channel expression in the human heart. Circ Arrhythm Electrophysiol 8: $1219-1227$

Li X, Pan K, Zhu D, Li Y, and Tao G (2016) Propofol postsynaptically suppresses stellate neuron excitability in the entorhinal cortex by influencing the $\mathrm{HCN}$ and TREK-2 channels. Neurosci Lett 619:54-59.

Li YD, Hong YF, Yusufuaji Y, Tang BP, Zhou XH, Xu GJ, Li JX, Sun L, Zhang JH, Xin Q, et al. (2015b) Altered expression of hyperpolarization-activated cyclic nucleotide-gated channels and microRNA-1 and -133 in patients with ageassociated atrial fibrillation. $\mathrm{Mol}$ Med Rep 12:3243-3248.

Li YD, Hong YF, Zhang Y, Zhou XH, Ji YT, Li HL, Hu GJ, Li JX, Sun L, Zhang JH, et al. (2014b) Association between reversal in the expression of hyperpolarizationactivated cyclic nucleotide-gated (HCN) channel and age-related atrial fibrillation. Med Sci Monit 20:2292-2297.

Li YL, Tran TP, Muelleman R, and Schultz HD (2008b) Blunted excitability of aortic baroreceptor neurons in diabetic rats: involvement of hyperpolarization-activated channel. Cardiovasc Res 79:715-721.

Li YL and Zheng H (2011) Angiotensin II-NADPH oxidase-derived superoxide mediates diabetes-attenuated cell excitability of aortic baroreceptor neurons. Am $J$ Physiol Cell Physiol 301:C1368-C1377.

Liang X, Wang G, Lin L, Lowe J, Zhang Q, Bu L, Chen Y, Chen J, Sun Y, and Evans SM (2013) HCN4 dynamically marks the first heart field and conduction system precursors. Circ Res 113:399-407.

Liao Z, Lockhead D, Larson ED, and Proenza C (2010) Phosphorylation and modulation of hyperpolarization-activated HCN4 channels by protein kinase A in the mouse sinoatrial node. J Gen Physiol 136:247-258.

Lillie C and Kobinger W (1986) Investigations into the bradycardic effects of UL-FS 49 (1,3,4,5-tetrahydro-7,8-dimethoxy-3-[3-[[2-(3,4-dimethoxyphenyl)ethyl] methylimino]propyl]-2H-3-benzazepin-2-on-hydrochloride) in isolated guinea pig atria. $J$ Cardiovasc Pharmacol 8:791-797.

Liu C, Xie C, Grant K, Su Z, Gao W, Liu Q, and Zhou L (2016) Patch-clamp fluorometry-based channel counting to determine HCN channel conductance. $J$ Gen Physiol 148:65-76.

Liu J, Noble PJ, Xiao G, Abdelrahman M, Dobrzynski H, Boyett MR, Lei M, and Noble D (2008) Role of pacemaking current in cardiac nodes: insights from a comparative study of sinoatrial node and atrioventricular node. Prog Biophys $\mathrm{Mol}$ Biol 96:294-304

Liu N, Zhang D, Zhu M, Luo S, and Liu T (2015) Minocycline inhibits hyperpolarization-activated currents in rat substantia gelatinosa neurons. Neuropharmacology $\mathbf{9 5}: 110-120$.
Liu Y-C, Wang Y-J, Wu P-Y, and Wu S-N (2009) Tramadol-induced block of hyperpolarization-activated cation current in rat pituitary lactotrophs. Naunyn Schmiedebergs Arch Pharmacol 379:127-135.

Lolicato M, Bucchi A, Arrigoni C, Zucca S, Nardini M, Schroeder I, Simmons K, Aquila M, DiFrancesco D, Bolognesi M, et al. (2014) Cyclic dinucleotides bind the C-linker of HCN4 to control channel cAMP responsiveness. Nat Chem Biol 10: $457-462$.

Lolicato M, Nardini M, Gazzarrini S, Möller S, Bertinetti D, Herberg FW, Bolognesi M, Martin H, Fasolini M, Bertrand JA, et al. (2011) Tetramerization dynamics of C-terminal domain underlies isoform-specific cAMP gating in hyperpolarizationactivated cyclic nucleotide-gated channels. J Biol Chem 286:44811-44820.

Lonardo G, Cerbai E, Casini S, Giunti G, Bonacchi M, Battaglia F, Fiorani B Stefano PL, Sani G, and Mugelli A (2004) Atrial natriuretic peptide modulates the hyperpolarization-activated current (If) in human atrial myocytes. Cardiovasc Res 63:528-536.

Lonardo G, Cerbai E, Casini S, Giunti G, Bonacchi M, Battaglia F, Fiorani B, Stefano PL, Sani G, and Mugelli A (2005) Pharmacological modulation of the hyperpolarization-activated current (If) in human atrial myocytes: focus on $G$ protein-coupled receptors. $J$ Mol Cell Cardiol 38:453-460.

López-González Z, Ayala-Aguilera C, Martinez-Morales F, Galicia-Cruz O, SalvadorHernández C, Pedraza-Chaverri J, Medeiros M, Hernández AM, and Escobar LI (2016) Immunolocalization of hyperpolarization-activated cationic HCN1 and HCN3 channels in the rat nephron: regulation of HCN3 by potassium diets. His tochem Cell Biol 145:25-40.

Lörincz A, Notomi T, Tamás G, Shigemoto R, and Nusser Z (2002) Polarized and compartment-dependent distribution of HCN1 in pyramidal cell dendrites. Nat Neurosci 5:1185-1193.

Ludwig A, Budde T, Stieber J, Moosmang S, Wahl C, Holthoff K, Langebartels A Wotjak C, Munsch T, Zong X, et al. (2003) Absence epilepsy and sinus dysrhythmia in mice lacking the pacemaker channel HCN2. EMBO $J$ 22:216-224.

Ludwig A, Zong X, Jeglitsch M, Hofmann F, and Biel M (1998) A family of hyperpolarization-activated mammalian cation channels. Nature 393:587-591.

Ludwig A, Zong X, Stieber J, Hullin R, Hofmann F, and Biel M (1999) Two pacemaker channels from human heart with profoundly different activation kinetics. $E M B O J$ 18:2323-2329.

Luján R, Albasanz JL, Shigemoto R, and Juiz JM (2005) Preferential localization of the hyperpolarization-activated cyclic nucleotide-gated cation channel subunit HCN1 in basket cell terminals of the rat cerebellum. Eur $J$ Neurosci 21: 2073-2082.

Lundström S, Twycross R, Mihalyo M, and Wilcock A (2010) Propofol. J Pain Symptom Manage 40:466-470.

Luo L, Chang L, Brown SM, Ao H, Lee DH, Higuera ES, Dubin AE, and Chaplan SR (2007) Role of peripheral hyperpolarization-activated cyclic nucleotide-modulated channel pacemaker channels in acute and chronic pain models in the rat. Neuroscience 144:1477-1485.

Maccaferri G, Mangoni M, Lazzari A, and DiFrancesco D (1993) Properties of the hyperpolarization-activated current in rat hippocampal CA1 pyramidal cells. $J$ Neurophysiol 69:2129-2136.

Macri V, Angoli D, and Accili EA (2012) Architecture of the HCN selectivity filter and control of cation permeation. Sci Rep 2:894.

Magee JC (1998) Dendritic hyperpolarization-activated currents modify the integrative properties of hippocampal CA1 pyramidal neurons. J Neurosci 18: $7613-7624$.

Magee JC (1999) Dendritic Ih normalizes temporal summation in hippocampal CA1 neurons. Nat Neurosci 2:848.

Maltsev VA and Lakatta EG (2012) The funny current in the context of the coupledclock pacemaker cell system. Heart Rhythm 9:302-307.

Maltsev VA, Rohwedel J, Hescheler J, and Wobus AM (1993) Embryonic stem cells differentiate in vitro into cardiomyocytes representing sinusnodal, atrial and ventricular cell types. Mech Dev 44:41-50.

Mangoni ME and Nargeot J (2008) Genesis and regulation of the heart automaticity. Physiol Rev 88:919-982.

Männikkö R, Elinder F, and Larsson HP (2002) Voltage-sensing mechanism is conserved among ion channels gated by opposite voltages. Nature 419:837-841.

Männikkö R, Pandey S, Larsson HP, and Elinder F (2005) Hysteresis in the voltage dependence of HCN channels: conversion between two modes affects pacemaker properties. J Gen Physiol 125:305-326.

Marger L, Mesirca P, Alig J, Torrente A, Dubel S, Engeland B, Kanani S, Fontanaud $\mathrm{P}$, Striessnig J, Shin HS, et al. (2011) Functional roles of $\mathrm{Ca}(\mathrm{v}) 1.3, \mathrm{Ca}(\mathrm{v}) 3.1$ and HCN channels in automaticity of mouse atrioventricular cells: insights into the atrioventricular pacemaker mechanism. Channels (Austin) 5:251-261.

Marionneau C, Couette B, Liu J, Li H, Mangoni ME, Nargeot J, Lei M, Escande D, and Demolombe S (2005) Specific pattern of ionic channel gene expression associated with pacemaker activity in the mouse heart. J Physiol 562:223-234.

Maroso M, Szabo GG, Kim HK, Alexander A, Bui AD, Lee SH, Lutz B, and Soltesz (2016) Cannabinoid control of learning and memory through HCN channels. Neuron 89:1059-1073.

Masi A, Narducci R, Landucci E, Moroni F, and Mannaioni G (2013) MPP(+) -dependent inhibition of Ih reduces spontaneous activity and enhances EPSP summation in nigral dopamine neurons. Br J Pharmacol 169:130-142.

Masi A, Narducci R, Resta F, Carbone C, Kobayashi K, and Mannaioni G (2015) Differential contribution of $\mathrm{Ih}$ to the integration of excitatory synaptic inputs in substantia nigra pars compacta and ventral tegmental area dopaminergic neurons. Eur J Neurosci 42:2699-2706.

Mattusch C, Kratzer S, Buerge M, Kreuzer M, Engel T, Kopp C, Biel M, Hammelmann V, Ying S-W, Goldstein PA, et al. (2015) Impact of hyperpolarization-activated, cyclic nucleotide-gated cation channel type 2 for the xenon-mediated anesthetic effect: evidence from in vitro and in vivo experiments. Anesthesiology 122: 1047-1059.

Mayer ML and Westbrook GL (1983) A voltage-clamp analysis of inward (anomalous) rectification in mouse spinal sensory ganglion neurones. J Physiol 340:19-45. 
McAlister FA, Wiebe N, Ezekowitz JA, Leung AA, and Armstrong PW (2009) Metaanalysis: $\beta$-blocker dose, heart rate reduction, and death in patients with heart failure. Ann Intern Med 150:784-794.

McCloskey KD, Toland HM, Hollywood MA, Thornbury KD, and McHale NG (1999) Hyperpolarisation-activated inward current in isolated sheep mesenteric lymphatic smooth muscle. J Physiol 521:201-211.

McClure KJ, Maher M, Wu N, Chaplan SR, Eckert IIIWA, Lee DH, Wickenden AD, Hermann M, Allison B, Hawryluk N, et al. (2011) Discovery of a novel series of selective HCN1 blockers. Bioorg Med Chem Lett 21:5197-5201.

McCormick DA and Pape HC (1990a) Noradrenergic and serotonergic modulation of a hyperpolarization-activated cation current in thalamic relay neurones. $J$ Physiol 431:319-342.

McCormick DA and Pape HC (1990b) Properties of a hyperpolarization-activated cation current and its role in rhythmic oscillation in thalamic relay neurones. $J$ Physiol 431:291-318.

McPherson GA and Angus JA (1989) Phentolamine and structurally related compounds selectively antagonize the vascular actions of the $\mathrm{K}+$ channel opener, cromromakalim. Br J Pharmacol 97:941-949.

Melchiorre M, Del Lungo M, Guandalini L, Martini E, Dei S, Manetti D, Scapecchi S, Teodori E, Sartiani L, Mugelli A, et al. (2010) Design, synthesis, and preliminary biological evaluation of new isoform-selective f-current blockers. J Med Chem $\mathbf{5 3}$ 6773-6777.

Melgari D, Brack KE, Zhang C, Zhang Y, El Harchi A, Mitcheson JS, Dempsey CE, Ng GA, and Hancox JC (2015) hERG potassium channel blockade by the HCN channel inhibitor bradycardic agent ivabradine. J Am Heart Assoc 4:e001813.

Meng Q-T, Xia Z-Y, Liu J, Bayliss DA, and Chen X (2011) Local anesthetic inhibits hyperpolarization-activated cationic currents. Mol Pharmacol 79:866-873.

Mesirca P, Alig J, Torrente AG, Müller JC, Marger L, Rollin A, Marquilly C, Vincent A, Dubel S, Bidaud I, et al. (2014) Cardiac arrhythmia induced by genetic silencing of 'funny' (f) channels is rescued by GIRK4 inactivation. Nat Commun 5:4664.

Michels G, Er F, Khan IF, Endres-Becker J, Brandt MC, Gassanov N, Johns DC, and Hoppe UC (2008) K+ channel regulator KCR1 suppresses heart rhythm by modulating the pacemaker current If. PLoS One 3:e1511.

Mika J (2008) Modulation of microglia can attenuate neuropathic pain symptoms and enhance morphine effectiveness. Pharmacol Rep 60:297-307.

Milanesi R, Baruscotti M, Gnecchi-Ruscone T, and DiFrancesco D (2006) Familial sinus bradycardia associated with a mutation in the cardiac pacemaker channel. $N$ Engl J Med 354:151-157.

Milano A, Vermeer AM, Lodder EM, Barc J, Verkerk AO, Postma AV, van der Bilt IA, Baars MJ, van Haelst PL, Caliskan K, et al. (2014) HCN4 mutations in multiple families with bradycardia and left ventricular noncompaction cardiomyopathy. $J$ Am Coll Cardiol 64:745-756.

Mistrík P, Mader R, Michalakis S, Weidinger M, Pfeifer A, and Biel M (2005) The murine HCN3 gene encodes a hyperpolarization-activated cation channel with slow kinetics and unique response to cyclic nucleotides. J Biol Chem 280 27056-27061.

Mistrík P, Pfeifer A, and Biel M (2006) The enhancement of HCN channel instantaneous current facilitated by slow deactivation is regulated by intracellular chloride concentration. Pflugers Arch 452:718-727.

Mobley AS, Miller AM, Araneda RC, Maurer LR, Müller F, and Greer CA (2010) Hyperpolarization-activated cyclic nucleotide-gated channels in olfactory sensory neurons regulate axon extension and glomerular formation. $J$ Neurosci $\mathbf{3 0}$ 16498-16508.

Möller S, Alfieri A, Bertinetti D, Aquila M, Schwede F, Lolicato M, Rehmann H, Moroni A, and Herberg FW (2014) Cyclic nucleotide mapping of hyperpolarizationactivated cyclic nucleotide-gated (HCN) channels. ACS Chem Biol 9:1128-1137.

Möller T, Bard F, Bhattacharya A, Biber K, Campbell B, Dale E, Eder C, Gan L, Garden GA, Hughes ZA, et al. (2016) Critical data-based re-evaluation of minocycline as a putative specific microglia inhibitor. Glia 64:1788-1794.

Momin A, Cadiou H, Mason A, and McNaughton PA (2008) Role of the hyperpolarization-activated current Ih in somatosensory neurons. J Physiol 586: 5911-5929.

Mommersteeg MT, Hoogaars WM, Prall OW, de Gier-de Vries C, Wiese C, Clout DE, Papaioannou VE, Brown NA, Harvey RP, Moorman AF, et al. (2007) Molecular pathway for the localized formation of the sinoatrial node. Circ Res 100:354-362.

Monfredi O and Boyett MR (2015) Sick sinus syndrome and atrial fibrillation in older persons - A view from the sinoatrial nodal myocyte. J Mol Cell Cardiol 83:88-100. Montalescot G, Sechtem U, Achenbach S, Andreotti F, Arden C, Budaj A, Bugiardini R, Crea F, Cuisset T, Di Mario C, et al.; Task Force Members; ESC Committee for Practice Guidelines; Document Reviewers (2013) 2013 ESC guidelines on the management of stable coronary artery disease: the Task Force on the management of stable coronary artery disease of the European Society of Cardiology. Eur Heart $J$ 34:2949-3003

Monteggia LM, Eisch AJ, Tang MD, Kaczmarek LK, and Nestler EJ (2000) Cloning and localization of the hyperpolarization-activated cyclic nucleotide-gated channel family in rat brain. Brain Res Mol Brain Res 81:129-139.

Moorman AF and Christoffels VM (2003) Cardiac chamber formation: development, genes, and evolution. Physiol Rev 83:1223-1267.

Moosmang S, Biel M, Hofmann F, and Ludwig A (1999) Differential distribution of four hyperpolarization-activated cation channels in mouse brain. Biol Chem 380: 975-980.

Moosmang S, Stieber J, Zong X, Biel M, Hofmann F, and Ludwig A (2001) Cellular expression and functional characterization of four hyperpolarization-activated pacemaker channels in cardiac and neuronal tissues. Eur $J$ Biochem 268 1646-1652.

Moroni A, Barbuti A, Altomare C, Viscomi C, Morgan J, Baruscotti M, and DiFrancesco D (2000) Kinetic and ionic properties of the human HCN2 pacemaker channel. Pflugers Arch 439:618-626.

Moser MB, Rowland DC, and Moser EI (2015) Place cells, grid cells, and memory. Cold Spring Harb Perspect Biol 7:a021808.
Much B, Wahl-Schott C, Zong X, Schneider A, Baumann L, Moosmang S, Ludwig A, and Biel M (2003) Role of subunit heteromerization and N-linked glycosylation in the formation of functional hyperpolarization-activated cyclic nucleotide-gated channels. J Biol Chem 278:43781-43786.

Müller F, Scholten A, Ivanova E, Haverkamp S, Kremmer E, and Kaupp UB (2003) HCN channels are expressed differentially in retinal bipolar cells and concentrated at synaptic terminals. Eur $J$ Neurosci 17:2084-2096.

Mummery C, Ward-van Oostwaard D, Doevendans P, Spijker R, van den Brink S, Hassink R, van der Heyden M, Opthof T, Pera M, de la Riviere AB, et al. (2003) Differentiation of human embryonic stem cells to cardiomyocytes: role of coculture with visceral endoderm-like cells. Circulation 107:2733-2740.

Munsch T and Pape HC (1999) Upregulation of the hyperpolarization-activated cation current in rat thalamic relay neurones by acetazolamide. J Physiol 519: 505-514.

Nakamura Y, Shi X, Numata T, Mori Y, Inoue R, Lossin C, Baram TZ, and Hirose S (2013) Novel HCN2 mutation contributes to febrile seizures by shifting the channel's kinetics in a temperature-dependent manner. PLoS One 8:e80376.

Nava C, Dalle C, Rastetter A, Striano P, de Kovel CG, Nabbout R, Cancès C, Ville D, Brilstra EH, Gobbi G, et al.; EuroEPINOMICS RES Consortium (2014) De novo mutations in HCN1 cause early infantile epileptic encephalopathy. Nat Genet 46: 640-645.

Nawathe PA, Kryukova Y, Oren RV, Milanesi R, Clancy CE, Lu JT, Moss AJ, Difrancesco D, and Robinson RB (2013) An LQTS6 MiRP1 mutation suppresses pacemaker current and is associated with sinus bradycardia. $J$ Cardiovasc Electrophysiol 24:1021-1027.

Neitz A, Mergia E, Imbrosci B, Petrasch-Parwez E, Eysel UT, Koesling D, and Mittmann T (2014) Postsynaptic NO/cGMP increases NMDA receptor currents via hyperpolarization-activated cyclic nucleotide-gated channels in the hippocampus. Cereb Cortex 24:1923-1936.

Neuhoff H, Neu A, Liss B, and Roeper J (2002) I(h) channels contribute to the dif ferent functional properties of identified dopaminergic subpopulations in the midbrain. J Neurosci 22:1290-1302.

Ng LC, Putrenko I, Baronas V, Van Petegem F, and Accili EA (2016) Cyclic purine and pyrimidine nucleotides bind to the HCN2 ion channel and variably promote C-terminal domain interactions and opening. Structure 24: 1629-1642.

Noam Y, Ehrengruber MU, Koh A, Feyen P, Manders EM, Abbott GW, Wadman WJ, and Baram TZ (2014) Filamin A promotes dynamin-dependent internalization of hyperpolarization-activated cyclic nucleotide-gated type 1 (HCN1) channels and restricts Ih in hippocampal neurons. J Biol Chem 289:5889-5903.

Nolan MF, Malleret G, Dudman JT, Buhl DL, Santoro B, Gibbs E, Vronskaya S, Buzsáki G, Siegelbaum SA, Kandel ER, et al. (2004) A behavioral role for dendritic integration: HCN1 channels constrain spatial memory and plasticity at inputs to distal dendrites of CA1 pyramidal neurons. Cell 119:719-732.

Nolan MF, Malleret G, Lee KH, Gibbs E, Dudman JT, Santoro B, Yin D, Thompson RF, Siegelbaum SA, Kandel ER, et al. (2003) The hyperpolarization-activated HCN1 channel is important for motor learning and neuronal integration by cerebellar Purkinje cells. Cell 115:551-564.

Notomi T and Shigemoto R (2004) Immunohistochemical localization of th channel subunits, HCN1-4, in the rat brain. J Comp Neurol 471:241-276.

Okamoto T, Harnett MT, and Morikawa H (2006) Hyperpolarization-activated cation current (Ih) is an ethanol target in midbrain dopamine neurons of mice. $\mathrm{J} \mathrm{Neu}$ rophysiol 95:619-626.

Oliphant CS, Owens RE, Bolorunduro OB, and Jha SK (2016) Ivabradine: a review of labeled and off-label uses. Am J Cardiovasc Drugs 16:337-347.

Opthof T (1998) The membrane current (I(f)) in human atrial cells: implications for atrial arrhythmias. Cardiovasc Res 38:537-540.

Paci M, Sartiani L, Del Lungo M, Jaconi M, Mugelli A, Cerbai E, and Severi S (2012) Mathematical modelling of the action potential of human embryonic stem cell derived cardiomyocytes. Biomed Eng Online 11:61.

Pape HC (1994) Specific bradycardic agents block the hyperpolarization-activated cation current in central neurons. Neuroscience 59:363-373.

Pape HC (1996) Queer current and pacemaker: the hyperpolarization-activated cation current in neurons. Annu Rev Physiol 58:299-327.

Pape HC and McCormick DA (1989) Noradrenaline and serotonin selectively modulate thalamic burst firing by enhancing a hyperpolarization-activated cation current. Nature 340:715-718.

Papp I, Holló K, and Antal M (2010) Plasticity of hyperpolarization-activated and cyclic nucleotid-gated cation channel subunit 2 expression in the spinal dorsal horn in inflammatory pain. Eur J Neurosci 32:1193-1201.

Papp I, Szucs P, Holló K, Erdélyi F, Szabó G, and Antal M (2006) Hyperpolarizationactivated and cyclic nucleotide-gated cation channel subunit 2 ion channels modulate synaptic transmission from nociceptive primary afferents containing substance P to secondary sensory neurons in laminae I-IIo of the rodent spinal dorsal horn. Eur J Neurosci 24:1341-1352.

Park K, Lee S, Kang SJ, Choi S, and Shin KS (2007) Hyperpolarization-activated currents control the excitability of principal neurons in the basolateral amygdala Biochem Biophys Res Commun 361:718-724.

Parker AR, Welch MA, Forster LA, Tasneem SM, Dubhashi JA, and Baro DJ (2017) SUMOylation of the hyperpolarization-activated cyclic nucleotide-gated channel 2 increases surface expression and the maximal conductance of the hyperpolarization-activated current. Front Mol Neurosci 9:168.

Parkis MA and Berger AJ (1997) Clonidine reduces hyperpolarization-activated inward current $\left(\mathrm{I}_{\mathrm{h}}\right)$ in rat hypoglossal motoneurons. Brain Res 769:108-118.

Paspalas CD, Wang M, and Arnsten AF (2013) Constellation of HCN channels and cAMP regulating proteins in dendritic spines of the primate prefrontal cortex: potential substrate for working memory deficits in schizophrenia. Cereb Cortex $\mathbf{2 3}$ 1643-1654

Pavlov I, Scimemi A, Savtchenko L, Kullmann DM, and Walker MC (2011) I(h)mediated depolarization enhances the temporal precision of neuronal integration. Nat Commun 2:199. 
Pérez O, Gay P, Franqueza L, Carrón R, Valenzuela C, Delpón E, and Tamargo J (1995a) Effects of the two enantiomers, S-16257-2 and S-16260-2, of a new bradycardic agent on guinea-pig isolated cardiac preparations. $\mathrm{Br} J$ Pharmacol 115 787-794.

Pérez O, Gay P, Franqueza L, Carrón R, Valenzuela C, Delpón E, and Tamargo J (1995b) Electromechanical effects of zatebradine on isolated guinea pig cardiac preparations. J Cardiovasc Pharmacol 26:46-54.

Pian P, Bucchi A, Decostanzo A, Robinson RB, and Siegelbaum SA (2007) Modulation of cyclic nucleotide-regulated HCN channels by PIP $(2)$ and receptors coupled to phospholipase C. Pflugers Arch 455:125-145.

Pian P, Bucchi A, Robinson RB, and Siegelbaum SA (2006) Regulation of gating and rundown of HCN hyperpolarization-activated channels by exogenous and endogenous PIP2. J Gen Physiol 128:593-604.

Pino R, Cerbai E, Calamai G, Alajmo F, Borgioli A, Braconi L, Cassai M, Montesi GF, and Mugelli A (1998) Effect of 5-HT4 receptor stimulation on the pacemaker current I(f) in human isolated atrial myocytes. Cardiovasc Res 40:516-522.

Piskorowski R, Santoro B, and Siegelbaum SA (2011) TRIP8b splice forms act in concert to regulate the localization and expression of HCN1 channels in CA1 pyramidal neurons. Neuron 70:495-509.

Ponikowski P, Voors AA, Anker SD, Bueno H, Cleland JG, Coats AJ, Falk V, González-Juanatey JR, Harjola VP, Jankowska EA, et al. (2016) 2016 ESC guidelines for the diagnosis and treatment of acute and chronic heart failure. Rev Esp Cardiol (Engl Ed) 69:1167.

Poolos NP, Bullis JB, and Roth MK (2006) Modulation of h-channels in hippocampal pyramidal neurons by p38 mitogen-activated protein kinase. $J$ Neurosci 26: 7995-8003.

Poolos NP, Migliore M, and Johnston D (2002) Pharmacological upregulation of h-channels reduces the excitability of pyramidal neuron dendrites. Nat Neurosci $\mathbf{5}$ : $767-774$.

Porciatti F, Pelzmann B, Cerbai E, Schaffer P, Pino R, Bernhart E, Koidl B, and Mugelli A (1997) The pacemaker current I(f) in single human atrial myocytes and the effect of $\beta$-adrenoceptor and A1-adenosine receptor stimulation. $B r J$ Pharmacol 122:963-969.

Proenza C, Angoli D, Agranovich E, Macri V, and Accili EA (2002) Pacemaker channels produce an instantaneous current. J Biol Chem 277:5101-5109.

Proenza C and Yellen G (2006) Distinct populations of HCN pacemaker channels produce voltage-dependent and voltage-independent currents. J Gen Physiol 127: $183-190$

Prole DL and Yellen G (2006) Reversal of HCN channel voltage dependence via bridging of the S4-S5 linker and Post-S6. J Gen Physiol 128:273-282.

Putrenko I and Schwarz SK (2011) Lidocaine blocks the hyperpolarization-activated mixed cation current, $\mathrm{I}_{\mathrm{h} \text { ) }}$ in rat thalamocortical neurons. Anesthesiology 115:822-835.

Qu J, Barbuti A, Protas L, Santoro B, Cohen IS, and Robinson RB (2001) HCN2 overexpression in newborn and adult ventricular myocytes: distinct effects on gating and excitability. Circ Res 89:E8-E14.

Qu J, Kryukova Y, Potapova IA, Doronin SV, Larsen M, Krishnamurthy G, Cohen IS, and Robinson RB (2004) MiRP1 modulates HCN2 channel expression and gating in cardiac myocytes. $J$ Biol Chem 279:43497-43502.

Qu Y, Whitaker GM, Hove-Madsen L, Tibbits GF, and Accili EA (2008) Hyperpolarization-activated cyclic nucleotide-modulated 'HCN' channels confer regular and faster rhythmicity to beating mouse embryonic stem cells. J Physio 586:701-716.

Raes A, Van de Vijver G, Goethals M, and van Bogaert PP (1998) Use-dependent block of Ih in mouse dorsal root ganglion neurons by sinus node inhibitors. $\mathrm{Br} J$ Pharmacol 125:741-750.

Rafizadeh S, Zhang Z, Woltz RL, Kim HJ, Myers RE, Lu L, Tuteja D, Singapuri A Bigdeli AAZ, Harchache SB, et al.(2014) Functional interaction with filamin A and intracellular $\mathrm{Ca} 2+$ enhance the surface membrane expression of a smallconductance Ca2+-activated K+ (SK2) channel. Proc Natl Acad Sci USA 111 9989-9994.

Ramakrishnan NA, Drescher MJ, Khan KM, Hatfield JS, and Drescher DG (2012) HCN1 and HCN2 proteins are expressed in cochlear hair cells: HCN1 can form a ternary complex with protocadherin $15 \mathrm{CD} 3$ and F-actin-binding filamin A or can interact with HCN2. J Biol Chem 287:37628-37646.

Rao K, Fisher ML, Robinson S, Shorofsky S, and Gottlieb SS (2007) Effect of chronic changes in heart rate on congestive heart failure. $J$ Card Fail 13.269-274.

Resta F, Masi A, Sili M, Laurino A, Moroni F, and Mannaioni G (2016) Kynurenic acid and zaprinast induce analgesia by modulating HCN channels through GPR35 activation. Neuropharmacology 108:136-143.

Ringkamp M, Tal M, Hartke TV, Wooten M, McKelvy A, Turnquist BP, Guan Y, Meyer RA, and Raja SN (2012) Local loperamide injection reduces mechanosensitivity of rat cutaneous, nociceptive C-fibers. PLoS One 7:e42105.

Robinson RB and Siegelbaum SA (2003) Hyperpolarization-activated cation currents from molecules to physiological function. Annu Rev Physiol 65:453-480.

Rocchetti M, Armato A, Cavalieri B, Micheletti M, and Zaza A (1999) Lidocaine inhibition of the hyperpolarization-activated current $(\mathrm{I}(\mathrm{f}))$ in sinoatrial myocytes. $J$ Cardiovasc Pharmacol 34:434-439.

Rodrigues AR and Oertel D (2006) Hyperpolarization-activated currents regulate excitability in stellate cells of the mammalian ventral cochlear nucleus. $J$ Neurophysiol 95:76-87.

Romanelli MN, Sartiani L, Masi A, Mannaioni G, Manetti D, Mugelli A, and Cerbai E (2016) HCN channels modulators: the need for selectivity. Curr Top Med Chem 16 1764-1791.

Rosen MR, Nargeot J, and Salama G (2012) The case for the funny current and the calcium clock. Heart Rhythm 9:616-618.

Saab CY (2012) Pain-related changes in the brain: diagnostic and therapeutic potentials. Trends Neurosci 35:629-637.

Saito Y, Inoue T, Zhu G, Kimura N, Okada M, Nishimura M, Kimura N, Murayama S, Kaneko S, Shigemoto R, et al. (2012) Hyperpolarization-activated cyclic nucleotide gated channels: a potential molecular link between epileptic seizures and $A \beta$ generation in Alzheimer's disease. Mol Neurodegener 7:50.
Sánchez-Alonso JL, Halliwell JV, and Colino A (2008) ZD 7288 inhibits T-type calcium current in rat hippocampal pyramidal cells. Neurosci Lett 439:275-280.

Santello M and Nevian T (2015) Dysfunction of cortical dendritic integration in neuropathic pain reversed by serotoninergic neuromodulation. Neuron 86:233-246. Santoro B, Chen S, Luthi A, Pavlidis P, Shumyatsky GP, Tibbs GR, and Siegelbaum SA (2000) Molecular and functional heterogeneity of hyperpolarization-activated pacemaker channels in the mouse CNS. $J$ Neurosci 20:5264-5275.

Santoro B, Grant SG, Bartsch D, and Kandel ER (1997) Interactive cloning with the SH3 domain of N-src identifies a new brain specific ion channel protein, with homology to eag and cyclic nucleotide-gated channels. Proc Natl Acad Sci USA 94: $14815-14820$

Santoro B, Liu DT, Yao H, Bartsch D, Kandel ER, Siegelbaum SA, and Tibbs GR (1998) Identification of a gene encoding a hyperpolarization-activated pacemaker channel of brain. Cell 93:717-729.

Santoro B, Piskorowski RA, Pian P, Hu L, Liu H, and Siegelbaum SA (2009) TRIP8b splice variants form a family of auxiliary subunits that regulate gating and trafficking of HCN channels in the brain. Neuron 62:802-813.

Santoro B, Wainger BJ, and Siegelbaum SA (2004) Regulation of HCN channel surface expression by a novel C-terminal protein-protein interaction. $J$ Neurosci 24:10750-10762.

Saponaro A, Pauleta SR, Cantini F, Matzapetakis M, Hammann C, Donadoni C, Hu L, Thiel G, Banci L, Santoro B, et al. (2014) Structural basis for the mutual antagonism of cAMP and TRIP8b in regulating HCN channel function. Proc Natl Acad Sci USA 111:14577-14582.

Sartiani L, Bettiol E, Stillitano F, Mugelli A, Cerbai E, and Jaconi ME (2007) Developmental changes in cardiomyocytes differentiated from human embryonic stem cells: a molecular and electrophysiological approach. Stem Cells 25:1136-1144.

Sartiani L, De Paoli P, Stillitano F, Aimond F, Vassort G, Mugelli A, and Cerbai E (2006) Functional remodeling in post-myocardial infarcted rats: focus on betaadrenoceptor subtypes. J Mol Cell Cardiol 40:258-266.

Sartiani L, Romanelli MN, Mugelli A, and Cerbai E (2015) Updates on HCN channels in the heart: function, dysfunction and pharmacology. Curr Drug Targets 16 868-876.

Sartiani L, Stillitano F, Luceri C, Suffredini S, Toti S, De Filippo C, Cuomo V, Tattoli M, Dolara P, Mugelli A, et al. (2010) Prenatal exposure to carbon monoxide delays postnatal cardiac maturation. Lab Invest 90:1582-1593.

Satin J, Kehat I, Caspi O, Huber I, Arbel G, Itzhaki I, Magyar J, Schroder EA, Perlman I, and Gepstein L (2004) Mechanism of spontaneous excitability in human embryonic stem cell derived cardiomyocytes. $J$ Physiol 559:479-496.

Satoh H (1991) Class III antiarrhythmic drugs (amiodarone, bretylium and sotalol) on action potentials and membrane currents in rabbit sino-atrial node preparations. Naunyn Schmiedebergs Arch Pharmacol 344:674-681.

Satoh T-O and Yamada M (2002) Multiple inhibitory effects of zatebradine (UL-FS 49) on the electrophysiological properties of retinal rod photoreceptors. Pflugers Arch 443:532-540.

Schirmer SH, Degen A, Baumhäkel M, Custodis F, Schuh L, Kohlhaas M, Friedrich E, Bahlmann F, Kappl R, Maack C, et al. (2012) Heart-rate reduction by If-channel inhibition with ivabradine restores collateral artery growth in hypercholesterolemic atherosclerosis. Eur Heart J 33:1223-1231.

Schnorr S, Eberhardt M, Kistner K, Rajab H, Käßer J, Hess A, Reeh P, Ludwig A, and Herrmann S (2014) HCN2 channels account for mechanical (but not heat) hyperalgesia during long-standing inflammation. Pain 155:1079-1090.

Schön C, Asteriti S, Koch S, Sothilingam V, Garcia Garrido M, Tanimoto N, Herms J, Seeliger MW, Cangiano L, Biel M, et al. (2016) Loss of HCN1 enhances disease progression in mouse models of CNG channel-linked retinitis pigmentosa and achromatopsia. Hum Mol Genet 25:1165-1175.

Schweizer PA, Yampolsky P, Malik R, Thomas D, Zehelein J, Katus HA, and Koenen M (2009) Transcription profiling of HCN-channel isotypes throughout mouse cardiac development. Basic Res Cardiol 104:621-629.

Scott S-P, Shea PW, and Dryer SE (2007) Mapping ligand interactions with the hyperpolarization activated cyclic nucleotide modulated (HCN) ion channel binding domain using a soluble construct. Biochemistry 46:9417-9431.

Scroggs RS, Todorovic SM, Anderson EG, and Fox AP (1994) Variation in IH, IIR, and ILEAK between acutely isolated adult rat dorsal root ganglion neurons of different size. J Neurophysiol 71:271-279.

Seifert R, Scholten A, Gauss R, Mincheva A, Lichter P, and Kaupp UB (1999) Molecular characterization of a slowly gating human hyperpolarization-activated channel predominantly expressed in thalamus, heart, and testis. Proc Natl Acad Sci USA 96:9391-9396.

Shah MM (2016) Hyperpolarization-activated cyclic nucleotide-gated channel currents in neurons. Cold Spring Harb Protoc 2016:pdb.top087346.

Shahi PK, Choi S, Zuo DC, Kim MY, Park CG, Kim YD, Lee J, Park KJ, So I, and Jun JY (2014) The possible roles of hyperpolarization-activated cyclic nucleotide channels in regulating pacemaker activity in colonic interstitial cells of Cajal. $J$ Gastroenterol 49:1001-1010.

Sheets PL, Suter BA, Kiritani T, Chan CS, Surmeier DJ, and Shepherd GM (2011) Corticospinal-specific HCN expression in mouse motor cortex: I(h)-dependent synaptic integration as a candidate microcircuit mechanism involved in motor control. J Neurophysiol 106:2216-2231.

Shin KS, Rothberg BS, and Yellen G (2001) Blocker state dependence and trapping in hyperpolarization-activated cation channels: evidence for an intracellular activation gate. J Gen Physiol 117:91-101.

Shin $\mathrm{M}$ and Chetkovich DM (2007) Activity-dependent regulation of $\mathrm{h}$ channel distribution in hippocampal CA1 pyramidal neurons. J Biol Chem 282:33168-33180.

Shirasaka T, Kannan H, and Takasaki M (2007) Activation of a G protein-coupled inwardly rectifying $\mathrm{K}+$ current and suppression of $\mathrm{Ih}$ contribute to dexmedetomidineinduced inhibition of rat hypothalamic paraventricular nucleus neurons. Anesthesiology 107:605-615.

Singh BN, Connolly SJ, Crijns HJGM, Roy D, Kowey PR, Capucci A, Radzik D, Aliot EM and Hohnloser SH; EURIDIS and ADONIS Investigators (2007) Dronedarone for maintenance of sinus rhythm in atrial fibrillation or flutter. N Engl J Med 357:987-999. 
Sobrado LF, Varone BB, Machado AD, Nearing BD, Zeng D, Belardinelli L, and Verrier RL (2013) Dronedarone's inhibition of If current is the primary mechanism responsible for its bradycardic effect. $J$ Cardiovasc Electrophysiol 24: 914-918

Später D, Abramczuk MK, Buac K, Zangi L, Stachel MW, Clarke J, Sahara M, Ludwig A, and Chien KR (2013) A HCN4+ cardiomyogenic progenitor derived from the first heart field and human pluripotent stem cells. Nat Cell Biol 15:1098-1106.

St Clair JR, Liao Z, Larson ED, and Proenza C (2013) PKA-independent activation of I(f) by cAMP in mouse sinoatrial myocytes. Channels (Austin) 7:318-321.

Stieber J, Herrmann S, Feil S, Löster J, Feil R, Biel M, Hofmann F, and Ludwig A (2003a) The hyperpolarization-activated channel HCN4 is required for the generation of pacemaker action potentials in the embryonic heart. Proc Natl Acad Sci USA 100:15235-15240.

Stieber J, Hofmann F, and Ludwig A (2004) Pacemaker channels and sinus node arrhythmia. Trends Cardiovasc Med 14:23-28.

Stieber J, Stöckl G, Herrmann S, Hassfurth B, and Hofmann F (2005) Functional expression of the human HCN3 channel. J Biol Chem 280:34635-34643.

Stieber J, Thomer A, Much B, Schneider A, Biel M, and Hofmann F (2003b) Molecular basis for the different activation kinetics of the pacemaker channels HCN2 and HCN4. J Biol Chem 278:33672-33680.

Stieber J, Wieland K, Stöckl G, Ludwig A, and Hofmann F (2006) Bradycardic and proarrhythmic properties of sinus node inhibitors. Mol Pharmacol 69:1328-1337. Stilli D, Sgoifo A, Macchi E, Zaniboni M, De Iasio S, Cerbai E, Mugelli A, Lagrasta C, Olivetti G, and Musso E (2001) Myocardial remodeling and arrhythmogenesis in moderate cardiac hypertrophy in rats. Am J Physiol Heart Circ Physiol 280: H142-H150.

Stillitano F, Lonardo G, Giunti G, Del Lungo M, Coppini R, Spinelli V, Sartiani L, Poggesi C, Mugelli A, and Cerbai E (2013) Chronic atrial fibrillation alters the functional properties of If in the human atrium. $J$ Cardiovasc Electrophysiol 24: $1391-1400$.

Stillitano F, Lonardo G, Zicha S, Varro A, Cerbai E, Mugelli A, and Nattel S (2008) Molecular basis of funny current (If) in normal and failing human heart. $\mathrm{J} \mathrm{Mol} \mathrm{Cell}$ Cardiol 45:289-299.

Suffredini S, Stillitano F, Comini L, Bouly M, Brogioni S, Ceconi C, Ferrari R, Mugelli A, and Cerbai E (2012) Long-term treatment with ivabradine in postmyocardial infarcted rats counteracts f-channel overexpression. $\mathrm{Br} J$ Pharmacol 165:1457-1466.

Suh BC and Hille B (2007) Regulation of KCNQ channels by manipulation of phosphoinositides. J Physiol 582:911-916.

Sundelacruz S, Levin M, and Kaplan DL (2009) Role of membrane potential in the regulation of cell proliferation and differentiation. Stem Cell Rev 5:231-246.

Sundelacruz S, Levin M, and Kaplan DL (2015) Comparison of the depolarization response of human mesenchymal stem cells from different donors. Sci Rep 5:18279.

Surges R, Brewster AL, Bender RA, Beck H, Feuerstein TJ, and Baram TZ (2006) Regulated expression of HCN channels and cAMP levels shape the properties of the h current in developing rat hippocampus. Eur J Neurosci 24:94-104.

Surges R, Freiman TM, and Feuerstein TJ (2003) Gabapentin increases the hyperpolarization-activated cation current Ih in rat CA1 pyramidal cells. Epilepsia 44:150-156.

Swynghedauw B (1999) Molecular mechanisms of myocardial remodeling. Physiol Rev 79:215-262.

Takasu K, Ono H, and Tanabe M (2010) Spinal hyperpolarization-activated cyclic nucleotide-gated cation channels at primary afferent terminals contribute to chronic pain. Pain 151:87-96

Takeda M, Ikeda M, Tanimoto T, Lipski J, and Matsumoto S (2002) Changes of the excitability of rat trigeminal root ganglion neurons evoked by $\alpha(2)$-adrenoreceptors. Neuroscience 115:731-741.

Tamura A, Ogura T, Uemura H, Reien Y, Kishimoto T, Nagai T, Komuro I, Miyazaki $\mathrm{M}$, and Nakaya H (2009) Effects of antiarrhythmic drugs on the hyperpolarizationactivated cyclic nucleotide-gated channel current. J Pharmacol Sci 110:150-159.

Tang B, Sander T, Craven KB, Hempelmann A, and Escayg A (2008) Mutation analysis of the hyperpolarization-activated cyclic nucleotide-gated channels HCN1 and HCN2 in idiopathic generalized epilepsy. Neurobiol Dis 29:59-70.

Taraska JW, Puljung MC, Olivier NB, Flynn GE, and Zagotta WN (2009) Mapping the structure and conformational movements of proteins with transition metal ion FRET. Nat Methods 6:532-537.

Thoby-Brisson M, Cauli B, Champagnat J, Fortin G, and Katz DM (2003) Expression of functional tyrosine kinase $B$ receptors by rhythmically active respiratory neurons in the pre-Bötzinger complex of neonatal mice. J Neurosci 23:7685-7689.

Thollon C, Cambarrat C, Vian J, Prost J-F, Peglion JL, and Vilaine JP (1994) Electrophysiological effects of S 16257, a novel sino-atrial node modulator, on rabbit and guinea-pig cardiac preparations: comparison with UL-FS 49. Br J Pharmacol 112:37-42.

Thon S, Schmauder R, and Benndorf K (2013) Elementary functional properties of single HCN2 channels. Biophys J 105:1581-1589.

Tibbs GR, Rowley TJ, Sanford RL, Herold KF, Proekt A, Hemmings Jr.HC, Andersen OS, Goldstein PA, and Flood PD (2013) HCN1 channels as targets for anesthetic and nonanesthetic propofol analogs in the amelioration of mechanical and thermal hyperalgesia in a mouse model of neuropathic pain. J Pharmacol Exp Ther $\mathbf{3 4 5}$ $363-373$

Torrente AG, Zhang R, Zaini A, Giani JF, Kang J, Lamp ST, Philipson KD, and Goldhaber JI (2015) Burst pacemaker activity of the sinoatrial node in sodiumcalcium exchanger knockout mice. Proc Natl Acad Sci USA 112:9769-9774.

Tran N, Proenza C, Macri V, Petigara F, Sloan E, Samler S, and Accili EA (2002) A conserved domain in the NH2 terminus important for assembly and functional expression of pacemaker channels. J Biol Chem 277:43588-43592.

Tsay D, Dudman JT, and Siegelbaum SA (2007) HCN1 channels constrain synaptically evoked $\mathrm{Ca} 2+$ spikes in distal dendrites of CA1 pyramidal neurons. Neuron $\mathbf{5 6}$ : $1076-1089$

Turley SL, Francis KE, Lowe DK, and Cahoon Jr.WD (2016) Emerging role of ivabradine for rate control in atrial fibrillation. Ther Adv Cardiovasc Dis 10:348-352.
Ulrich D (2002) Dendritic resonance in rat neocortical pyramidal cells. J Neurophysiol 87:2753-2759.

Valenzuela C, Delpón E, Franqueza L, Gay P, Pérez O, Tamargo J, and Snyders DJ (1996) Class III antiarrhythmic effects of zatebradine: time-, state-, use-, and voltage-dependent block of hKv1.5 channels. Circulation 94:562-570.

Van Bogaert PP and Pittoors F (2003) Use-dependent blockade of cardiac pacemaker current $\left(\mathrm{I}_{f}\right)$ by cilobradine and zatebradine. Eur $J$ Pharmacol 478:161-171.

van Kempen M, van Ginneken A, de Grijs I, Mutsaers N, Opthof T, Jongsma H, and van der Heyden M (2003) Expression of the electrophysiological system during murine embryonic stem cell cardiac differentiation. Cell Physiol Biochem 13:263-270.

VanSchouwen B, Akimoto M, Sayadi M, Fogolari F, and Melacini G (2015) Role of dynamics in the autoinhibition and activation of the hyperpolarization-activated cyclic nucleotide-modulated (HCN) ion channels. J Biol Chem 290:17642-17654.

Vasilyev DV, Shan Q, Lee Y, Mayer SC, Bowlby MR, Strassle BW, Kaftan EJ, Rogers KE, and Dunlop J (2007) Direct inhibition of Ih by analgesic loperamide in rat DRG neurons. $J$ Neurophysiol 97:3713-3721.

Vasilyev DV, Shan QJ, Lee YT, Soloveva V, Nawoschik SP, Kaftan EJ, Dunlop J, Mayer SC, and Bowlby MR (2009) A novel high-throughput screening assay for HCN channel blocker using membrane potential-sensitive dye and FLIPR. J Biomol Screen 14:1119-1128.

Verkerk AO and Wilders R (2014) Pacemaker activity of the human sinoatrial node: effects of HCN4 mutations on the hyperpolarization-activated current. Europace 16:384-395.

Verrier RL, Bonatti R, Silva AF, Batatinha JA, Nearing BD, Liu G, Rajamani S, Zeng $\mathrm{D}$, and Belardinelli L (2014) If inhibition in the atrioventricular node by ivabradine causes rate-dependent slowing of conduction and reduces ventricular rate during atrial fibrillation. Heart Rhythm 11:2288-2296.

Verrier RL, Silva AF, Bonatti R, Batatinha JA, Nearing BD, Liu G, Rajamani S, Zeng $\mathrm{D}$, and Belardinelli L (2015) Combined actions of ivabradine and ranolazine reduce ventricular rate during atrial fibrillation. J Cardiovasc Electrophysiol 26:329-335

Verrier RL, Sobrado MF, Pagotto VP, Kanas AF, Machado AD, Varone BB, Sobrado LF, Nearing BD, Zeng D, and Belardinelli L (2013) Inhibition of I(f) in the atrioventricular node as a mechanism for dronedarone's reduction in ventricular rate during atrial fibrillation. Heart Rhythm 10:1692-1697.

Vinogradova TM, Brochet DX, Sirenko S, Li Y, Spurgeon H, and Lakatta EG (2010) Sarcoplasmic reticulum $\mathrm{Ca} 2+$ pumping kinetics regulates timing of local $\mathrm{Ca} 2+$ releases and spontaneous beating rate of rabbit sinoatrial node pacemaker cells. Circ Res 107:767-775.

Viscomi C, Altomare C, Bucchi A, Camatini E, Baruscotti M, Moroni A and DiFrancesco D (2001) C terminus-mediated control of voltage and cAMP gating of hyperpolarization-activated cyclic nucleotide-gated channels. $J$ Biol Chem 276:29930-29934.

Wahl-Schott C and Biel M (2009) HCN channels: structure, cellular regulation and physiological function. Cell Mol Life Sci 66:470-494.

Wahl-Schott C, Fenske S, and Biel M (2014) HCN channels: new roles in sinoatrial node function. Curr Opin Pharmacol 15:83-90.

Wainger BJ, DeGennaro M, Santoro B, Siegelbaum SA, and Tibbs GR (2001) Molecular mechanism of cAMP modulation of $\mathrm{HCN}$ pacemaker channels. Nature 411 $805-810$

Wang J, Chen S, Nolan MF, and Siegelbaum SA (2002) Activity-dependent regulation of HCN pacemaker channels by cyclic AMP: signaling through dynamic allosteric coupling. Neuron 36:451-461.

Wang J, Chen S, and Siegelbaum SA (2001) Regulation of hyperpolarizationactivated HCN channel gating and cAMP modulation due to interactions of $\mathrm{COOH}$ terminus and core transmembrane regions. J Gen Physiol 118:237-250.

Wang K, Xue T, Tsang SY, Van Huizen R, Wong CW, Lai KW, Ye Z, Cheng L, Au KW, Zhang J, et al. (2005) Electrophysiological properties of pluripotent human and mouse embryonic stem cells. Stem Cells 23:1526-1534.

Wang M, Ramos BP, Paspalas CD, Shu Y, Simen A, Duque A, Vijayraghavan S, Brennan A, Dudley A, Nou E, et al. (2007) Alpha2A-adrenoceptors strengthen working memory networks by inhibiting cAMP-HCN channel signaling in prefrontal cortex. Cell 129:397-410.

Wang WT, Wan YH, Zhu JL, Lei GS, Wang YY, Zhang P, and Hu SJ (2006) Thetafrequency membrane resonance and its ionic mechanisms in rat subicular pyramidal neurons. Neuroscience 140:45-55.

Wang Y and Hill JA (2010) Electrophysiological remodeling in heart failure. $J M o$ Cell Cardiol 48:619-632.

Weisbrod D, Peretz A, Ziskind A, Menaker N, Oz S, Barad L, Eliyahu S, ItskovitzEldor J, Dascal N, Khananshvili D, et al. (2013) SK4 Ca2+ activated K+ channel is a critical player in cardiac pacemaker derived from human embryonic stem cells. Proc Natl Acad Sci USA 110:E1685-E1694.

Wemhöner K, Silbernagel N, Marzian S, Netter MF, Rinné S, Stansfeld PJ, and Decher N (2012) A leucine zipper motif essential for gating of hyperpolarization-activated channels. J Biol Chem 287:40150-40160.

Weng X, Smith T, Sathish J, and Djouhri L (2012) Chronic inflammatory pain is associated with increased excitability and hyperpolarization-activated current (Ih)

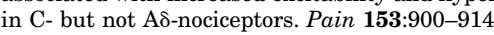

Whitaker GM, Angoli D, Nazzari H, Shigemoto R, and Accili EA (2007) HCN2 and HCN4 isoforms self-assemble and co-assemble with equal preference to form functional pacemaker channels. J Biol Chem 282:22900-22909.

White SM and Claycomb WC (2005) Embryonic stem cells form an organized, functional cardiac conduction system in vitro. Am J Physiol Heart Circ Physiol 288: H670-H679.

Wicks NL, Wong T, Sun J, Madden Z, and Young EC (2011) Cytoplasmic cAMPsensing domain of hyperpolarization-activated cation (HCN) channels uses two structurally distinct mechanisms to regulate voltage gating. Proc Natl Acad Sci USA 108:609-614

Wilkars W, Wollberg J, Mohr E, Han M, Chetkovich DM, Bähring R, and Bender RA (2014) Nedd4-2 regulates surface expression and may affect N-glycosylation of hyperpolarization-activated cyclic nucleotide-gated (HCN)-1 channels. FASEB J 28:2177-2190. 
Williams AD, Jung S, and Poolos NP (2015) Protein kinase C bidirectionally modulates Ih and hyperpolarization-activated cyclic nucleotide-gated (HCN) channel surface expression in hippocampal pyramidal neurons. $J$ Physiol 593:2779-2792.

Williams SR and Stuart GJ (2000) Site independence of EPSP time course is mediated by dendritic $\mathrm{I}(\mathrm{h})$ in neocortical pyramidal neurons. $J$ Neurophysiol 83: $3177-3182$.

Wilson CM and Farrell AP (2013) Pharmacological characterization of the heartbeat in an extant vertebrate ancestor, the Pacific hagfish, Eptatretus stoutii. Comp Biochem Physiol A Mol Integr Physiol 164:258-263.

$\mathrm{Wu} \mathrm{JY}$ and Cohen IS (1997) Tyrosine kinase inhibition reduces $\mathrm{i}(\mathrm{f})$ in rabbit sinoatrial node myocytes. Pflugers Arch 434:509-514.

Wu X, Liao L, Liu X, Luo F, Yang T, and Li C (2012) Is ZD7288 a selective blocker of hyperpolarization-activated cyclic nucleotide-gated channel currents? Channels (Austin) 6:438-442.

Xu X, Marni F, Wu S, Su Z, Musayev F, Shrestha S, Xie C, Gao W, Liu Q, and Zhou L (2012) Local and global interpretations of a disease-causing mutation near the ligand entry path in hyperpolarization-activated cAMP-gated channel. Structure 20:2116-2123

Xu X, Vysotskaya ZV, Liu Q, and Zhou L (2010) Structural basis for the cAMPdependent gating in the human HCN4 channel. J Biol Chem 285:37082-37091.

Xue L, Li Y, Han X, Yao L, Yuan J, Qin W, Liu F, and Wang H (2012a) Investigation of hyperpolarization-activated cyclic nucleotide-gated channels in interstitial cells of Cajal of human bladder. Urology 80:224.e13-224.e18.

Xue T, Marbán E, and Li RA (2002) Dominant-negative suppression of HCN1- and HCN2-encoded pacemaker currents by an engineered HCN1 construct: insights into structure-function relationships and multimerization. Circ Res 90:1267-1273.

Xue WN, Wang Y, He SM, Wang XL, Zhu JL, and Gao GD (2012b) SK- and h-current contribute to the generation of theta-like resonance of rat substantia nigra pars compacta dopaminergic neurons at hyperpolarized membrane potentials. Brain Struct Funct 217:379-394

Yagi J and Sumino R (1998) Inhibition of a hyperpolarization-activated current by clonidine in rat dorsal root ganglion neurons. J Neurophysiol 80:1094-1104.

Yamada Y, Kinoshita H, Kuwahara K, Nakagawa Y, Kuwabara Y, Minami T, Yamada C, Shibata J, Nakao K, Cho K, et al. (2014) Inhibition of N-type Ca2+ channels ameliorates an imbalance in cardiac autonomic nerve activity and prevents lethal arrhythmias in mice with heart failure. Cardiovasc Res 104:183-193.

Yanagi K, Takano M, Narazaki G, Uosaki H, Hoshino T, Ishii T, Misaki T, and Yamashita JK (2007) Hyperpolarization-activated cyclic nucleotide-gated channels and T-type calcium channels confer automaticity of embryonic stem cellderived cardiomyocytes. Stem Cells 25:2712-2719.

Yanagihara K and Irisawa H (1980) Inward current activated during hyperpolarization in the rabbit sinoatrial node cell. Pflugers Arch 385:11-19.

Yanagihara K, Noma A, and Irisawa H (1980) Reconstruction of sino-atrial node pacemaker potential based on the voltage clamp experiments. Jpn J Physiol 30: 841-857.

Yang Q, Kuzyk P, Antonov I, Bostwick CJ, Kohn AB, Moroz LL, and Hawkins RD (2015) Hyperpolarization-activated, cyclic nucleotide-gated cation channels in Aplysia: contribution to classical conditioning. Proc Natl Acad Sci USA 112: 16030-16035.

Yang Y-C, Meng Q-T, Pan X, Xia Z-Y, and Chen X-D (2014) Dexmedetomidine produced analgesic effect via inhibition of HCN currents. Eur J Pharmacol $\mathbf{7 4 0}$ 560-564.

Yao H, Donnelly DF, Ma C, and LaMotte RH (2003) Upregulation of the hyperpolarization-activated cation current after chronic compression of the dorsal root ganglion. J Neurosci 23:2069-2074.

Ye B and Nerbonne JM (2009) Proteolytic processing of HCN2 and co-assembly with HCN4 in the generation of cardiac pacemaker channels. $J$ Biol Chem 284 25553-25559.

Yeon K-Y, Chung G, Kim YH, Hwang JH, Davies AJ, Park M-K, Ahn DK, Kim JS, Jung S.J, and Oh SB (2011) Eugenol reverses mechanical allodynia after peripheral nerve injury by inhibiting hyperpolarization-activated cyclic nucleotide-gated (HCN) channels. Pain 152:2108-2116.

Yi F, Danko T, Botelho SC, Patzke C, Pak C, Wernig M, and Südhof TC (2016) Autism-associated SHANK3 haploinsufficiency causes Ih channelopathy in human neurons. Science 352:aaf2669.

Ying S-W, Abbas SY, Harrison NL, and Goldstein PA (2006) Propofol block of I(h) contributes to the suppression of neuronal excitability and rhythmic burst firing in thalamocortical neurons. Eur J Neurosci 23:465-480.

Ying SW, Jia F, Abbas SY, Hofmann F, Ludwig A, and Goldstein PA (2007) Dendritic HCN2 channels constrain glutamate-driven excitability in reticular thalamic neurons. $J$ Neurosci 27:8719-8732.
Ying SW, Tibbs GR, Picollo A, Abbas SY, Sanford RL, Accardi A, Hofmann F, Ludwig A and Goldstein PA (2011) PIP2-mediated HCN3 channel gating is crucial for rhythmic burst firing in thalamic intergeniculate leaflet neurons. J Neurosci 31: 10412-10423.

Yoshimoto R, Iwasaki S, Takago H, Nakajima T, Sahara Y, and Kitamura K (2015) Developmental increase in hyperpolarization-activated current regulates intrinsic firing properties in rat vestibular ganglion cells. Neuroscience 284:632-642.

Young GT, Emery EC, Mooney ER, Tsantoulas C, and McNaughton PA (2014) Inflammatory and neuropathic pain are rapidly suppressed by peripheral block of hyperpolarisation-activated cyclic nucleotide-gated ion channels. Pain 155: 1708-1719.

Yu H, Wu J, Potapova I, Wymore RT, Holmes B, Zuckerman J, Pan Z, Wang H, Shi W, Robinson RB, et al. (2001) MinK-related peptide 1: a beta subunit for the HCN ion channel subunit family enhances expression and speeds activation. Circ Res $\mathbf{8 8}$ : E84-E87.

Yu HD, Xia S, Zha CQ, Deng SB, Du JL, and She Q (2015) Spironolactone regulates HCN protein expression through micro-RNA-1 in rats with myocardial infarction. $J$ Cardiovasc Pharmacol 65:587-592.

Yu HG, Lu Z, Pan Z, and Cohen IS (2004) Tyrosine kinase inhibition differentially regulates heterologously expressed HCN channels. Pflugers Arch 447:392-400.

Zagotta WN, Olivier NB, Black KD, Young EC, Olson R, and Gouaux E (2003) Structural basis for modulation and agonist specificity of HCN pacemaker channels. Nature 425:200-205.

Zaza A (2016) Electrophysiology meets geometry. Europace 18:317.

Zaza A and Lombardi F (2001) Autonomic indexes based on the analysis of heart rate variability: a view from the sinus node. Cardiovasc Res 50:434-442.

Zaza A, Rocchetti M, and DiFrancesco D (1996) Modulation of the hyperpolarizationactivated current $(\mathrm{I}(\mathrm{f})$ ) by adenosine in rabbit sinoatrial myocytes. Circulation 94 734-741.

Zhang H and Dougherty PM (2014) Enhanced excitability of primary sensory neurons and altered gene expression of neuronal ion channels in dorsal root ganglion in paclitaxel-induced peripheral neuropathy. Anesthesiology 120:1463-1475.

Zhang H, Sun AY, Kim JJ, Graham V, Finch EA, Nepliouev I, Zhao G, Li T, Lederer WJ, Stiber JA, et al. (2015) STIM1-Ca ${ }^{2+}$ signaling modulates automaticity of the mouse sinoatrial node. Proc Natl Acad Sci USA 112:E5618-E5627.

Zhang Y, Liu Y, Qu J, Hardy A, Zhang N, Diao J, Strijbos PJ, Tsushima R, Robinson $\mathrm{RB}$, Gaisano HY, et al. (2009) Functional characterization of hyperpolarizationactivated cyclic nucleotide-gated channels in rat pancreatic beta cells. $J$ Endocrinol 203:45-53.

Zhang Y, Zhang N, Gyulkhandanyan AV, Xu E, Gaisano HY, Wheeler MB, and Wang Q (2008) Presence of functional hyperpolarisation-activated cyclic nucleotide-gated channels in clonal alpha cell lines and rat islet alpha cells. Diabetologia 51: $2290-2298$.

Zhou C, Ke B, Zhao Y, Liang P, Liao D, Li T, Liu J, and Chen X (2015a) Hyperpolarization-activated cyclic nucleotide-gated channels may contribute to regional anesthetic effects of lidocaine. Anesthesiology 122:606-618.

Zhou C, Liang P, Liu J, Ke B, Wang X, Li F, Li T, Bayliss DA, and Chen X (2015b) HCN1 channels contribute to the effects of amnesia and hypnosis but not immobility of volatile anesthetics. Anesth Analg 121:661-666.

Zhou L and Siegelbaum SA (2007) Gating of HCN channels by cyclic nucleotides: residue contacts that underlie ligand binding, selectivity, and efficacy. Structure 15:655-670.

Zhou M and MacKinnon R (2004) A mutant KcsA K(+) channel with altered conduction properties and selectivity filter ion distribution. J Mol Biol 338:839-846. Zicha S, Fernández-Velasco M, Lonardo G, L'Heureux N, and Nattel S (2005) Sinus node dysfunction and hyperpolarization-activated (HCN) channel subunit remodeling in a canine heart failure model. Cardiovasc Res 66:472-481.

Zolles G, Klöcker N, Wenzel D, Weisser-Thomas J, Fleischmann BK, Roeper J, and Fakler B (2006) Pacemaking by HCN channels requires interaction with phosphoinositides. Neuron 52:1027-1036.

Zolles G, Wenzel D, Bildl W, Schulte U, Hofmann A, Müller CS, Thumfart JO, Vlachos A, Deller T, Pfeifer A, et al. (2009) Association with the auxiliary subunit PEX5R Trip8b controls responsiveness of HCN channels to cAMP and adrenergic stimulation. Neuron 62:814-825.

Zong X, Eckert C, Yuan H, Wahl-Schott C, Abicht H, Fang L, Li R, Mistrik P, Gerstner A, Much B, et al. (2005) A novel mechanism of modulation of hyperpolarization-activated cyclic nucleotide-gated channels by Src kinase. J Biol Chem 280:34224-34232.

Zong X, Krause S, Chen C-C, Krüger J, Gruner C, Cao-Ehlker X, Fenske S, WahlSchott C, and Biel M (2012) Regulation of hyperpolarization-activated cyclic nucleotide-gated (HCN) channel activity by cCMP. J Biol Chem 287:26506-26512. 